\section{EUROSPINE 2017 SCIENTIFIC PROGRAMME QUICK FIRE PRESENTATIONS}

Dublin/ EURO :

\section{EUROSPINE 2017}

Scientific Programme

Quick Fire presentations

\section{DEFORMITY/GROWING SPINE}

\section{QF1 \\ MINIMAL CLINICALLY IMPORTANT DIFFERENCE (MCID) FOR CPCHILD QUESTIONNAIRE FOLLOWING SCOLIOSIS SURGERY IN CEREBRAL PALSY}

Luigi Aurelio Nasto, Firoz Miyanji, Paul Sponseller, Tracey Bastrom, Suken Shah, Amer Samdani, Harry Shufflebarger, Burt Yaszay

Department of Orthopaedic Surgery, BC Children's Hospital, Vancouver, BC, Canada

Background/introduction: Patient reported outcomes have evolved to include an understanding of the patient's perspective of change. Minimal clinically important difference (MCID) is defined as the minimum amount of change important to the patient/caregiver in any given patient reported outcome measure tool. CPCHILD is designed to measure HRQoL in cerebral palsy (CP) patients and has been recently used to study outcomes following scoliosis surgery in this population. However, the MCID threshold for this questionnaire as it relates to surgical outcomes remains unknown.

Purpose of the study: The aim of this study was to define a MCID threshold score of the CPCHILD in CP patients undergoing scoliosis surgery with minimum 2-year f/u.

Materials and methods: An anchor-based method utilizing a prospective, multicenter $\mathrm{CP}$ scoliosis surgical database was conducted. All consecutive $\mathrm{CP}$ patients with $\geq 2$ years $\mathrm{f} / \mathrm{u}$ who underwent deformity correction were analyzed. CPCHILD scores were collected at baseline and 2-years post-op. Caregivers were asked (anchor questions) to rate on a five level Likert-type scale the effect of surgery with regard to improvement in quality of life (QOL), comfort (COM), ease of care (EOC), and general health (GH) at 2-year f/u. Change in CPCHILD total score (TS) was then compared to caregiver responses to the anchors. Linear and logistic regression models were used to calculate the MCID.
Results: 132 patients were included. Mean age was $14 \pm 2.6$ years, majority were GMFCS 5 (80\%). CPCHILD TS score changes at 2 years (follow-up minus baseline) per each anchor question are shown in the Table. MCID for a significant improvement in QOL, COM, and EOC was $10(\mathrm{p}=0.016), 15.8(\mathrm{p}=0.01)$, and $6.8(\mathrm{p}=0.022)$ points, respectively. GH anchor did not reach statistical significance. In patients who reached the MCID the odds of improvement with surgery was 1.77 fold higher in QOL, 4.9 fold higher in COM, and 1.48 fold higher in EOC than those that did not. The probability of patients "improving" after surgery if the MCID was reached was 63.9, 83, and 59.5\% in the anchors QOL, COM, and EOC, respectively.

Conclusion: No univocal estimate of MCID value for CPCHILD can be given. However, utilizing an anchor-based method we provide MCID values of $10,15.8$, and 6.8 in the CPCHILD TS to predict a significant improvement in QOL, COM, and EOC at 2-years post-op. Disclosures: Author 1: none; Author 2: grants/research support: Depuy Synthes Spine, consultant: Depuy Synthes Spine; Author 3: grants/research support: depuy synthes spine, royalties: Depuy Synthes Spine, Globus; Author 4: grants/research support: Research grants from DePuy Synthes Spine and from K2M to Setting Scoliosis Straight Foundation for Harms Study Group research efforts; Author 6: consultant: DePuy Synthes Spine, Ethicon, Globus Medical, Misonix, Stryker, Zimmer Biomet; Author 7: grants/research support: DePuy Synths, consultant: DePuy, K2M, royalties: DePuy, K2M; Author 8: grants/research support: Depuy Synthes, K2M, consultant: Depuy Synthes, K2M, Nuvasive, Globus, royalties: K2M, Orthopediatrics, other financial report: Styker; Author 9: grants/research support: DePuy Spine; K2M, Inc; EOS Imaging; OREF, SRS, employee: Setting Scoliosis Straight; Author 10: grants/research support: Grant from DePuy Synthes to Setting Scoliosis Straight Foundation in support of the Harms Study Group's research; AO Spine; John and Marcella Fox Fund Grant; OREF, consultant: DePuy Synthes; Ethicon; stock/shareholder: Paradigm Spine; Spine Search, royalties: DePuy Synthes, employee: Mount Sinai Hospital.

\begin{tabular}{|c|c|c|c|c|c|c|c|c|c|}
\hline \multicolumn{10}{|c|}{ CPCHILD Total Score Change } \\
\hline \multirow[b]{2}{*}{ Effect of Surgery } & \multirow[b]{2}{*}{$N$} & \multicolumn{2}{|l|}{ QOL } & \multicolumn{3}{|c|}{ сом } & \multicolumn{3}{|c|}{ EOC } \\
\hline & & Avg & SD & N & Avg & SD & $N$ & Avg & SD \\
\hline Improved a lot & 98 & +9.53 & (13.16) & 92 & +10.07 & (13.28) & 65 & +10.10 & (13.75) \\
\hline Improved a little & 20 & +0.50 & (11.59) & 21 & +4.91 & $(9.94)$ & 36 & +5.15 & (12.46) \\
\hline No change & 4 & +3.91 & (11.98) & 7 & -1.23 & (15.54) & 12 & +4.75 & (13.45) \\
\hline Deteriorated a little & 8 & -5.06 & $(17.57)$ & 10 & -10.48 & $(10.69)$ & 14 & -1.31 & (12.98) \\
\hline Deteriorated a lot & 2 & -5.82 & (10.82) & 1 & -13.48 & $(-)$ & 3 & -8.73 & $(5.36)$ \\
\hline \multirow[t]{2}{*}{ Missing } & 0 & & & \multicolumn{3}{|l|}{1} & \multicolumn{3}{|l|}{2} \\
\hline & MCID & \multicolumn{2}{|c|}{$95 \% \mathrm{CI}$} & MCID & \multirow{2}{*}{\multicolumn{2}{|c|}{$\begin{array}{c}95 \% \mathrm{Cl} \\
53615312\end{array}$}} & MCID & \multicolumn{2}{|c|}{$95 \% \mathrm{CI}$} \\
\hline Linear regression & +9.99 & \multicolumn{2}{|c|}{$(4.69,15.30)$} & +10.34 & & & +6.48 & (1.80, & \\
\hline \multirow[t]{3}{*}{ Logistic regression } & +1.0 & \multicolumn{2}{|c|}{$\begin{array}{l}+6.0 \% \text { chances of } \\
\text { improvement }\end{array}$} & +1.0 & \multicolumn{2}{|c|}{$\begin{array}{l}+6.4 \% \text { chances } \\
\text { of improvement }\end{array}$} & +1.0 & \multicolumn{2}{|c|}{$\begin{array}{l}+3.8 \% \text { chances } \\
\text { of improvement }\end{array}$} \\
\hline & +5.0 & \multicolumn{2}{|c|}{$\begin{array}{c}+35.0 \% \text { chances of } \\
\text { improvement }\end{array}$} & +5.0 & \multicolumn{2}{|c|}{$\begin{array}{c}+37.7 \% \text { chances of } \\
\text { improvement }\end{array}$} & +5.0 & \multicolumn{2}{|c|}{$\begin{array}{l}+20.9 \% \text { chances of } \\
\text { improvement }\end{array}$} \\
\hline & +10.0 & \multicolumn{2}{|c|}{$\begin{array}{c}+82.2 \% \text { changes of } \\
\text { improvement }\end{array}$} & +10.0 & \multicolumn{2}{|c|}{$\begin{array}{c}+89.6 \% \text { chances of } \\
\text { improvement }\end{array}$} & +10.0 & \multicolumn{2}{|c|}{$\begin{array}{c}+46.2 \% \text { chances of } \\
\text { improvement }\end{array}$} \\
\hline
\end{tabular}




\section{QF2}

\section{RADIATION EXPOSURE DURING THE COURSE OF TREATMENT FOR EARLY-ONSET SCOLIOSIS- LONGITUDINAL PILOT STUDY}

\author{
En Xie, Dingjun Hao
}

Hong-Hui Hospital, Xi'an Jiaotong University College of Medicine, Xi'an, Shaanxi, China

Study design: Retrospective consecutive case series. Objectives: To estimate the amount of ionizing radiation (IR) exposure in early-Onset Scoliosis for early-Onset Scoliosis.

Summary of background data: There is abundant evidence of the health hazards attributed to IR exposure. However, no studies have estimated the amount of IR exposure in early-onset scoliosis.

Materials and methods: A consecutive single-center series of earlyOnset Scoliosis patients were retrospectively reviewed. Of 372 total patients, 337 had a minimum 2-year follow-up and complete records available for analysis. All spine-related IR imaging studies are excluding interpretative fluoroscopy were tabulated and early-Onset Scoliosis estimated based on historical controls in millisieverts $(\mathrm{mSv})$. Results: Initial X-ray evaluation for scoliosis was made at a mean age of 5.7 years (range 2-9 year). Mean radiographic period was 11 years (range 2-18). There was a statistically significant inverse correlation between patient age at time of initial IR and total mean IR $(\mathrm{p}<0.05)$. Total IR was 3.4 times greater than that of estimated background radiation $(2.7 \mathrm{mSv}$ per year). Annual IR after the first diagnosed yearly averaged $7.71 \mathrm{mSv}$ (range $2.17-15.07$ ). Overall, 79\% of total IR were attributed to X-rays and $21 \%$ from computed tomography. Conclusions: Compared to the general public. Early-Onset Scoliosis patients had 3.7 times more IR than the estimated background radiation for the same duration of time. Younger patients had significantly higher IR doses. This study stresses the importance of recognizing the amount of IR used in the management of early-Onset Scoliosis patients and its potential long-term risks.

Disclosures: Author 1: none; Author 2: none.

\section{QF3}

\section{WHERE IS THE AXIS OF ROTATION IN THORACIC IDIOPATHIC SCOLIOSIS?}

Rob Brink, Tom Schlösser, Marijn vanStralen, Koen Vincken, Moyo Kruyt, Lin Shi, Winnie Chu, René Castelein, Jack Cheng

Department of Orthopaedic Surgery, University Medical Center Utrecht, Utrecht, The Netherlands

Introduction: Adolescent idiopathic scoliosis (AIS) is a three-dimensional (3-D) deformity of the spine, in which the apical region is mostly rotated and translated away from the midline. Unfortunately, its etiopathogenesis is still elusive. Quantification of the rotational mechanism could help to understand the 3-D development of the deformity and to improve treatment methods.

Purpose of the study: To quantify the longitudinal axis of rotation of individual segments in the primary thoracic curve in patients with AIS.

Materials and methods: From an existing database, 70 high-resolution CT scans $\left(\mathrm{Cobb} 46^{\circ}-109^{\circ}\right)$, acquired for spinal navigation, were included. Complete 3-D reconstructions were acquired, using a previously validated technique. Vertebral vectors, in a 3-D coordinate system, were used to quantify the location of the longitudinal axis of rotation for each vertebra in the transverse plane in relation to the midsagittal plane of the sacral plate. Rotation radius was defined as the distance between the center of the spinal canal and the longitudinal axis of rotation (Fig. 1).

Results: For all levels, the center of rotation was localized far posterior to the spine and was closer to the spine in the apical region (rotation radius \pm standard deviation): apex, $11.5 \pm 5.6 \mathrm{~cm}$ vs two levels below $(14.1 \pm 8.2 \mathrm{~cm})$ and above $(15.8 \pm 8.5 \mathrm{~cm})$ the apex ( $\mathrm{p}<0.001)$. Larger coronal Cobb angle corresponded with increased rotation and a smaller rotational radius $(\mathrm{R} 2=0.55$; $\mathrm{p}<0.001)$.

Conclusion: Our results confirm that in AIS, the vertebrae rotate away from its original position around a longitudinal axis localized far posterior to the spine and far remote from where it has been located in the normal spine, as described in previous studies. This could suggest an extra-spinal biomechanical driver for rotation in the scoliotic mechanism. The findings could serve as important reference for intraoperative derotation maneuvers.

Disclosures: Author 1: none; Author 2: grants/research support: AO Spine European Young Researcher Award; Author 3: stock/shareholder: MRIguidance BV; Author 4: none; Author 5: none; Author 7: none; Author 8: grants/research support: K2M; Author 9: none.

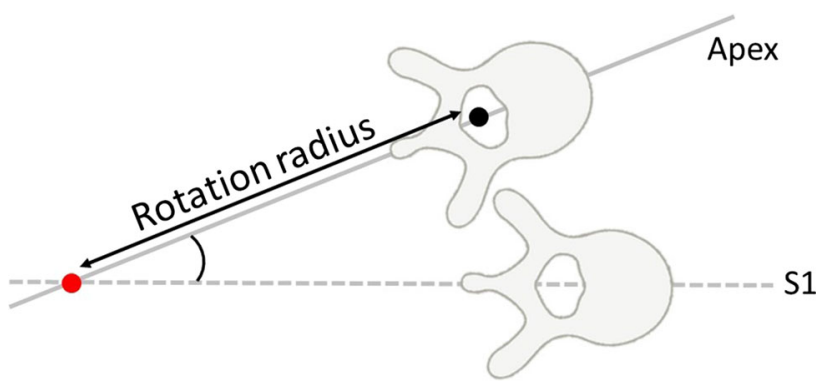

QF4

THE EFFICACY OF SERIAL CORRECTIVE CASTING FOR THE COMPENSATORY CURVE OF CONGENITAL SCOLIOSIS

Shimei Tanida, Bungo Otsuki, Shunsuke Fujibayashi, Shuichi Matsuda, Noriaki Kawakami

Department of Orthopaedic Surgery, Graduate School of Medicine, Kyoto University, Kyoto, Japan

Introduction: In the treatment strategy for congenital scoliosis, it is important to prevent the compensatory curve, which forms above or below the congenital curve, from progressing in respect of shortening fusion segments in the future. Surgical intervention was delayed until the patient grew approximately 5 years old or $100 \mathrm{~cm}$ in height, if possible, because of the existing implant size. Therefore, in principle, we had conducted the serial corrective casting (Risser-Cotrel cast) with patient awake for congenital scoliosis with the compensatory curve forming in the thoracolumbar or lumbar lesions as "time-saving" strategy. In this study, we retrospectively investigated whether the serial corrective casting was effective in prevention the compensatory curve in the congenital scoliosis from progressing until surgical intervention was performed.

Methods: The consecutive 26 patients, who were diagnosed as congenital scoliosis with $>30^{\circ}$ of the congenital curve during follow-up period and treated with serial corrective casting for minimum 1 year, were included. We measured the Cobb angle of the congenital and the compensatory curve in the thoracolumbar or lumbar lesion, and T1-12 height in anteroposterior X-ray on standing position. 
Results: The average age at initial casting was 2.9 years (range 1.2-6.1 years). The average duration of the serial corrective casting was 3.1 years (range 1.0-6.9 years). The initial corrective casting significantly reduced both the congenital and the compensatory curve $(\mathrm{p}<0.05)$ (Figure). During the treatment period, the corrective casting was treated 6.0 times (range 1-15 times) per patient. The compensatory curve remained stable throughout the treatment [pretreatment Cobb angle; $30.8^{\circ}$ (range $12^{\circ}-75^{\circ}$ ), Cobb angle at the end of the treatment; $29.1^{\circ}$ (range $\left.7^{\circ}-60^{\circ}\right)$ ] (Figure). The Congenital curve also remained stable throughout the treatment [pretreatment Cobb angle; $52.0^{\circ}$ (range $30^{\circ}-82^{\circ}$ ), Cobb angle at the end of the treatment; $52.1^{\circ}$ (range $28^{\circ}-$ $\left.100^{\circ}\right)$ ]. The Cobb angle of the congenital and compensatory curves didn't significantly change during the treatment $(\mathrm{p}>0.05)$. During the treatment period, T1-12 height increased $2.9 \pm 1.7 \mathrm{~cm}$.

Discussion: Although the corrective casting helps delay surgical intervention in early-onset idiopathic scoliosis, the efficacy for the congenital scoliosis remained unestablished. In this study, the corrective casting prevented the compensatory curve forming in the thoracolumbar or lumbar lesions from progressing, and spared the thoracic height during the treatment period. Furthermore, unexpectedly, the propression of the congenital curve was also prevented. These results suggested that the corrective casting was effective as not only "time-saving", but also " growth-sparing" strategy in congenital scoliosis.

Disclosures: Author 1: none; Author 2: none; Author 3: none; Author 4: none; Author 5: consultant: Medtronic, Johnson \& Johnson, Kisco, other financial report: NPO Japan Spinal Deformity Institute.

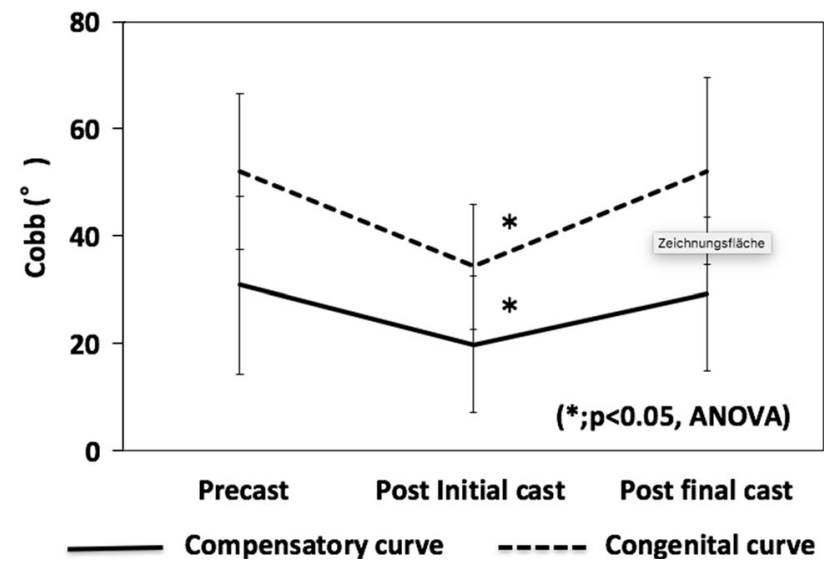

QF5

DOES PROXIMAL HOOK FIXATION PREVENT MID-TERM
PROXIMAL JUNCTIONAL KYPHOSIS IN PATIENTS OPERATED FOR ADOLESCENT IDIOPATHIC SCOLIOSIS?

Felipe Rioseco, Alejandro Gomez-Rice, Javier Pizones, Felisa Sánchez-Mariscal, Jose Miguel Sánchez-Marquez, Mar Pérez MartínBuitrago, Lorenzo Zúñiga, Nicomedes Fernández-Baíllo,

Francisco Javier Pérez-Grueso, Enrique Izquierdo

Hospital Universitario La Paz, Hospital Universitario de Getafe, Madrid, Spain

Introduction: It has been suggested that the use of hooks in the upper instrumented level may prevent PJK in Adolescent Idiopathic Scoliosis (AIS) compared with the use of pedicle screws. However, no $\mathrm{mid} /$ long term studies have assessed this effect. The aim of the study was to analyze the mid-term PJK prevention comparing hooks vs screws as upper anchors in AIS.
Methods: We conducted a retrospective study analyzing thoracic AIS curves (Lenke 1-4) operated on by posterior approach, with an upper instrumented level in T6 or above, and minimum 5-year follow-up. We compared those patients who had screws as proximal anchorage vs those with hooks. Proximal Junctional Angle (PJA), T1-T5 kyphosis, T5-T12 kyphosis and Spinosacral Angle (SSA) were measured in preoperative, postoperative and final lateral X-rays. Revision surgeries and complications were evaluated. PJK was defined as a PJA of at least $10^{\circ}$ between the lower end plate of the uppermost instrumented vertebrae and the upper end plate of the 2 immediately superior vertebrae, and at least $10^{\circ}$ of progression from the previous measurement.

Results Fifty patients met inclusion criteria. 27 with hooks at the proximal fusion level, 23 with screws. The mean follow-up was 78.5months (60-240). 46 were female, and 4 were males. Mean age at surgery 14 years (11-18). No significant difference was found regarding T1-T5 kyphosis, T5-T12 kyphosis, preoperative SSA, postoperative PJA and final PJA. "Hooks" group had significantly greater T5-T12 postoperative kyphosis than the "screws" group, $18^{\circ}$ $\left(7^{\circ}-37^{\circ}\right)$ vs $10^{\circ}\left(2^{\circ}-22^{\circ}\right) ; \mathrm{p}=0.000$. Five patients $(10 \%)$ developed a PJK (3 from "screws", 2 from "hooks"; no statistically significant association) showing a final PJA of $15^{\circ}$ (14-28). No patient required surgical revision for PJK. Patients with PJK had a preoperative Cobb angle significantly smaller $50^{\circ}(45-52)$ vs $59.75^{\circ}(47-87)(\mathrm{p}<0.05)$. 8 patients required surgical revision (seven from the "hooks" group, one from the "screws" group; $\mathrm{p}<0.05)$. Three patients required surgical revision for proximal hook dislodgement.

Conclusion: In our study population proximal hooks did not prevent mid-term PJK when compared to screws. Patients with proximal hooks had a greater postoperative kyphosis, but a greater number of revision surgeries. No patient with PJK needed revision surgery.

Disclosures: Author 1: none; Author 2: consultant: Medtronic, Stryker; Author 3: consultant: DePuy Synthes Johnson and Johnson; Author 4: none; Author 5: none; Author 6: grants/research support: DepuySynthes; Author 7: none; Author 9: grants/research support: DEPUY SINTHES, consultant: K2M. DEPUY SINTHES; Author 10: consultant: PRIM.

\begin{tabular}{|c|c|c|c|}
\hline & Preoperative & Postoperative & Final \\
\hline T1-T5 Kyphosis & $10.5 \div(2.37)$ & $14.5^{2}(2.36)$ & $15^{2}(2-36)$ \\
\hline T5-T12 Kyphosis & $18^{9}(3-47)$ & $16.5^{\circ}(2-37)$ & $15^{\circ}(2-38)$ \\
\hline $\begin{array}{l}\text { Proximal } \\
\text { Junctional Angle }\end{array}$ & $69(0-23)$ & $7^{2}(0-20)$ & $92(0-28)$ \\
\hline$\overline{S S A}$ & $138^{\circ}(120-160)$ & $135^{\circ}(121-164)$ & $135^{\circ}(119-164)$ \\
\hline $\begin{array}{l}\text { Main Curve Cobb } \\
\text { Angle }\end{array}$ & $58^{2}(45-87)$ & $20^{\circ}(2-42)$ & $21^{2}(2-40)$ \\
\hline
\end{tabular}

QF6

EXTREME CORONAL CORRECTION AND AXIAL DEROTATION PROMPT TO POST-OPERATIVE SHOULDER IMBALANCE IN IMMATURE AIS PATIENTS

Vicente García, Jesús Burgos, Carlos Barrios, Miguel Antón, Eduardo Hevia, Ignacio Sanpera, Gabriel Pizá, Miguel Castro

Department of Orthopaedic Surgery, Hospital Mancha Centro. Alcázar de San Juan, Ciudad Real; Hospital Ramón y Cajal, Madrid; Universidad Católica De Valencia, Valencia; Hospital Ramón Y Cajal, Madrid, España; Hospital Central La Fraternidad-Muprespa, Madrid; Hospital Universitari Son Espases, Islas Baleares; Hospital Son Espases. Palma De Mallorca, Islas Baleares; Hospital Materno Infantil Teresa Herrera. La Coruña, Spain

Introduction: Postop shoulder imbalance is a major concern in AIS. Literature is scarce analyzing the influence of the amount of coronal 
correction and axial derotation on the shoulders balance. This study analyzed these radiologic parameters in a series of mature and immature AIS.

Purpose of the study: The purpose of this study is to analize the relationship between postop abnormal shoulder balance and extreme coronal correction and axial derotation, particularly in immature AIS patients.

Materials and methods: Retrospective radiologic study from a prospective database. 30 AIS patients with Lenke type 1A curves that underwent all pedicle screw corrective surgery by vertebral coplanar alignment were included. Patient demographics, correction of MT curve, axial apical rotation, Risser sign, and XR shoulder inequality were measured at preop, immediate postop, and 3, 6, 12 and 24-month FU. A modified 4-grade Upasani's scale was used to evaluate the position of screws.

Results: Patients had a mean age of 13 years, $55.9^{\circ}$ preop Cobb, and $23^{\circ}$ apical rotation. 13 cases were immature. The left shoulder was sloped a mean of $17 \mathrm{~mm}$ in height. The overall coronal correction was $85.2 \%$ (18 cases surpassing $80 \%$ ). Mean apex derotation reached $7.7^{\circ}$ $(66.5 \%)$. In the immediate postop, left shoulder elevation showed positive correlation with the magnitude of MT curve correction $(\mathrm{r}=0.568 ; \mathrm{p}<0.01)$, and inverse correlation with apical derotation $(\mathrm{r}=-0.495 ; \mathrm{p}<0.05)$. Immature patients exhibited a more intense left shoulder elevation in the immediate postop period $(\mathrm{p}<0.05)$. Incomplete correction of the postop shoulder elevation was observed from 6-month FU $(7.6 \mathrm{~mm}$ in cases with $>80 \%$ coronal correction, and $1.1 \mathrm{~mm}$ in $<80 \%)$.

Conclusions: Extreme coronal MT curve correction $\left(>80^{\circ}\right)$ and severe apical vertebrae derotation were statistically associated with greater postop shoulder imbalance. Surgeons should avoid extreme coronal correction and axial derotation, particularly in immature AIS patients.

Disclosures: Author 1: none; Author 2: none; Author 3: none; Author 4: none; Author 5: none; Author 6: none; Author 7: consultant: Medtronic; Author 8: none.

\section{QF7 \\ THE OUTCOME OF VARIOUS SURGICAL TREATMENTS OF SPINAL STENOSIS IN ACHONDROPLASIA: A SYSTEMATIC REVIEW}

\section{Zaid Abual-Rub, Jennifer Campbell, Ghulam Abbas, Almas Khan}

Leeds General Infirmary, Leeds, UK

Background: Introduction: Individuals with achondroplasia can be associated with spinal stenosis due to short pedicles and reduced interpedicular distance. Approximately $20-30 \%$ of patients are symptomatic, and up to $10 \%$ might require treatment. The aim of this study was to assess the reliability and validity of the evidence for surgical treatments to address spinal stenosis in achondroplasia with respect to functional outcomes.

Methods: We performed a systematic search of the online databases of PubMed/Medline, Embase, Cochrane Library, AMED, and CINAHL to identify studies that evaluated the treatment outcomes of spinal stenosis using decompression alone vs decompression and fusion in children and adults with achondroplasia.

Results: We identified 9 retrospective studies involving 268 patients. The average age was 30.8 years, with a mean follow up of 4.5 years. The mean duration of stenotic symptoms prior to intervention was 3.9 years. In seven out of nine studies, patients underwent decompression only; two studies performed decompression only, as well as, decompression and instrumented fusion in the same cohort.
Patient: Reported outcome measures (PROMs) were reported in three out of nine studies. Symptoms fully or partially resolved in $48.5 \%$ of patients; recurrence of symptoms was reported in $11 \%$. Intra-operative durotomy rate was $16.7 \%$, while the total re-operation rate in this population was $38 \%$. In one study of spinal stenosis in a paediatric population, post-operative kyphotic deformity developed in $100 \%$ of patients, and all required instrumented stabilisation.

Conclusion(s): Studies were of noticeable heterogeneity in reporting their surgical and functional outcomes. Surgical intervention carries a significant risk of re-operation and associated complications in this population. Based on the available literature, there is insufficient evidence to suggest superiority of one surgical treatment option over another. However, in the paediatric population an instrumented fusion should be considered due to the risk of post-operative kyphotic deformity.

Implications: Multi-centre prospective collection of pre-identified data on specific surgical outcomes and validated PROMs is required to provide evidence to inform future practice.

Disclosures: Author 1: none; Author 2: none; Author 3: none; Author 4: consultant: Stryker

\section{QF8}

\section{THORACOLUMBAR ADULT SCOLIOSIS PATIENTS: 2-YEAR SURGICAL RESULTS OF A PROSPECTIVE COHORT}

Javier Pizones, Mar Pérez Martín-Buitrago, Francisco javier sánchez Pérez-Grueso, Mariana Díaz-Almirón, Ahmet Alanay, Ibrahim Obeid, Emre Acaroglu, Frank Kleinstueck, Ferran Pellise, European Spine Study Group ESSG

\section{Hospital Universitario La Paz, Madrid, Spain}

Introduction: Despite the existing literature on surgical treatment for adult spinal deformity in general, there is a lack of specific information regarding thoracolumbar (TL) deformity, even though it is the most prevalent spinal deformity and receives the highest percentage of surgical treatment in the adult population. The aim of the study was to describe the radiographic and clinical results of thoracolumbar adult scoliosis surgery.

Methods: Retrospective descriptive analysis of prospectively collected data from an adult deformity multicenter database. Only patients operated for main thoracolumbar/lumbar (TL) curves in our multicenter database were included. Paired-t test analysis of radiographic and clinical results at a minimum 2-year follow-up was performed.

Results: A total of 175 patients met inclusion criteria. Mean age was $52.4 \pm 19$ years. See table for preoperative and postoperative data. At 2 years, the TL Cobb achieved $60 \%$ of correction, and the compensatory thoracic curve $49 \%$, while the lumbar mismatch and the SVA were secondarily corrected. The HRQoL questionnaires (COMI, ODI, SF-36 and SRS-22) improved in all domains, especially in function, pain and self-image. The number of fused levels was $10.9 \pm 3.5$, blood loss was $1522 \pm 1190 \mathrm{ml}$, surgical time was $303 \pm 128 \mathrm{~min}$, and hospital stay was $11 \pm 9$ days. 76 patients underwent osteotomies, and 77 were instrumented to the pelvis. 101 patients suffered complications (49 minor, 52 major). There were 43 revision surgeries in those 2 years.

Conclusions: Surgery for adult thoracolumbar scoliosis at 2 years achieved $60 \%$ of coronal correction, slightly improving sagittal alignment and lumbar mismatch. The best clinical improvements were obtained in function, pain, and self-image, which were above the minimal clinical important difference. However, surgical costs 
were high, with a $57 \%$ complication rate and $24 \%$ of revision surgeries.

Disclosures: Author 1: grants/research support: DePuy Synthes Johnson and Johnson, consultant: DePuy Synthes Johnson and Johnson; Author 2: grants/research support: DepuySynthes; Author 3: grants/research support: DEPUY SINTHES, consultant: K2M. DEPUY SINTHES; Author 4: none; Author 5: grants/research support: Depuy\&Synthes, consultant: Medtronic; Author 6: grants/ research support: DEPUY SYNTHES SPINE, consultant: DEPUY SYNTHES, MEDTRONIC, royalties: ALPHATEC SPINE, SPINEART, CLARAIANCE; Author 7: grants/research support: Depuy Synthes, Medtronic, stock/shareholder: IncredX, royalties: Zimmer Biomet, AOSpine; Author 8: grants/research support: Depuy-Synthes; Author 9: grants/research support: DePuy Synthes Spine, Medtronic, consultant: DePuy Synthes Spine, Biomet; Author 10: grants/research support: DePuy-synthes.

\section{QF9 \\ IMPACT OF THREE COLUMN OSTEOTOMY ON HEALTH RELATED QUALITY OF LIFE MEASURES IN PATIENTS WITH ADULT SPINAL DEFORMITY: A SYSTEMATIC REVIEW AND META-ANALYSIS}

Muralidharn Venkatesan, Jagdeep Atwal, Dritan Pasku, Sarang Sapare, Nasir Quraishi

\section{The Centre for Spinal Studies and Surgery, Nottingham, UK}

Background: Three-column osteotomies (3-CO) have gained popularity in the last decade as surgical intervention for adult spinal deformity (ASD). 3-CO in the form of either Pedicle subtraction osteotomy (PSO) or vertebral column resection (VCR) is required to achieve adequate correction for severe and rigid deformity. The aim of the 3-CO is to correct and provide a balanced spine thereby improving pain and function. Studies reporting improvement in health related quality of life with validated outcome measures after 3-CO surgery are sparse and currently consist of of small series.

Objective: Systematic review and meta-analysis to evaluate improvement in health related quality of life measures following 3-CO for adult spinal deformity.

Methods: Two independent reviewers conducted systematic review of English literature between period 1996 and 2016 for articles reporting outcome of 3-CO in patients with ASD. Review was conducted according to PRISMA (Preferred Reporting Items for Systematic reviews and Meta-Analyses) guidelines using a PRISMA checklist. Inclusion criteria were studies reporting patient reported outcome (ODI, SRS 22, SF36) after 3-CO surgery for adult spine deformity patients (18 years or older) with a minimum follow-up of 1 year. A random effect model was used for meta-analysis to combine the studies for each outcome and forest plots were prepared. Outcomes were expressed as mean difference (MD)

Results: Eight studies with 364 PSO and 63 VCR were included for the meta-analysis. The pooled mean for ODI was -15.1 (95\% CI -18.1 to -12.5 ) with negative value indicating mean drop in ODI scores from the pre-operative to the post-operative state and the mean effect size for SRS-22 was 0.61 with $95 \%$ CI $0.49-0.73$.

Conclusion: Three-column osteotomy surgery improve quality of life in patients with adult spinal deformity.

Disclosures: Author 1: none; Author 2: none; Author 3: none; Author 4: none; Author 5: consultant: Medtronic.

\section{QF10}

IMPACT OF EARLY MAJOR RESOLVED COMPLICATIONS ON 2-YEAR FOLLOW-UP OUTCOME FOLLOWING ADULT SPINAL DEFORMITY SURGERY

Susana Núñez-Pereira, Alba Vila-Casademunt, Montse DomingoSàbat, Sleiman Haddad, Ibrahim Obeid, Ahmet Alanay, Emre Acaroglu, Francisco Sánchez Pérez-Grueso, Frank Kleinstück, Ferran Pellisé, European Spine Study Group, ESSG

\section{Vall d'Hebron Research Institute, Barcelona, Spain}

Introduction: Major complications are a great concern following adult spine deformity (ASD) surgery. Despite being managed properly, they might leave a lasting impact on health related quality of life (HRQL) long after their resolution. We aimed to investigate the impact of early major complications resolving within the first 6 month after ASD surgery on the 2-year outcome.

Hypothesis: The impairment caused by a major complication can be detected at 2-years after index surgery, despite resolving within the first 6 months.

Methods: Two groups of consecutive surgical patients were extracted from a prospective multicenter ASD database. The complication Group (G1) included patients who suffered a major complication that resolved within the first 6 months after surgery. Patients who suffered further complications diagnosed or resolved beyond the 6-month mark were excluded. This insured an 18-month complication-free interval before reaching the study endpoint. Patients with no major complication during the entire follow-up composed the control group (G2). An analysis of covariance adjusting for the preoperative baseline values was performed to compare the improvement on each HRQL measure between the two groups at 2-years.

Results: Out of the 402 eligible patients, 175 met the inclusion criteria and had complete HRQL data at 2-years (24 G1, 151 G2). G1 patients were older, had greater deformity and worse HRQL at baseline (Table). They suffered a total of 27 major complications resolved by 6 months $(8$ implant related, 5 deep surgical site infections, 5 medical complications, 2 motor deficits with full recovery, 2 PJK, 5 other). 19 unplanned surgeries were performed in G1 (18 revisions, 1 cholecystectomy). At 2-years, and after adjusting for preoperative baseline values, G1 patients had worse outcome compared to G2. Namely, they had worse ODI (5.98 points more than G2 (SE 3.03); $\mathrm{p}=0.05$ ), SRS-22function (0.36 points less (SE 0.13); $\mathrm{p}=0.01)$, SF-36 PCS (4.07 points less (SE 1.93); $\mathrm{p}=0.04)$ and SRS-22subtotal (0.16 points less (SE 0.13); $\mathrm{p}=0.22$ ).

Conclusion: The impact of a major complication after ASD surgery long outlives its resolution and can still be detected after 18 month of complication-free interval. Patients suffering an early and resolved complication experience significantly less improvement (SRS-22function, ODI, SF-36 PCS) at 2 years after index surgery compared to their uncomplicated counterparts.

Disclosures: Author 1: grants/research support: Depuy Synthes; Author 2: grants/research support: Depuy Synthes Spine; Author 3: grants/research support: DePuy-Synthes; Author 4: none; Author 5: grants/research support: DEPUY SYNTHES, consultant: DEPUY SYNTHES, MEDTRONIC, royalties: ALPHATEC, SPINEART, CLARIANCE; Author 6: grants/research support: Depuy, consultant: Medtronic; Author 7: grants/research support: Depuy Synthes, Medtronic, stock/shareholder: IncredX, royalties: Zimmer Biomet, AOSpine; Author 8: grants/research support: DEPUY sinthes, consultant: K2M. DEPUY SINTHES; Author 9: grants/research support: Depuy-Synthes; Author 10: grants/research support: DePuy Synthes Spine, Medtronic, consultant: DePuy Synthes Spine, Biomet. 


\begin{tabular}{|c|c|c|c|c|c|c|}
\hline \multirow{4}{*}{ Gender } & \multirow{2}{*}{ G1 } & $F:$ & \multicolumn{4}{|l|}{$13(54.2 \%)$} \\
\hline & & M & \multicolumn{4}{|l|}{$11(45.8 \%)$} \\
\hline & \multirow{2}{*}{$\mathrm{G} 2$} & F: & \multicolumn{4}{|c|}{$118(78.2 \%)$} \\
\hline & & $M$ & \multicolumn{4}{|c|}{$33(21.8 \%)$} \\
\hline \multirow{2}{*}{ Age } & $\mathrm{G} 1$ & \multirow{2}{*}{\multicolumn{5}{|c|}{ 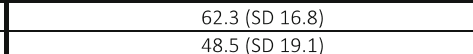 }} \\
\hline & G2 & & & & & \\
\hline \multirow{6}{*}{ ASA } & \multirow{3}{*}{ G1 } & I: & \multicolumn{4}{|l|}{$6(25.0 \%)$} \\
\hline & & II: & \multirow{2}{*}{\multicolumn{4}{|c|}{$\frac{14(58.3 \%)}{4(16.7 \%)}$}} \\
\hline & & III & & & & \\
\hline & \multirow{3}{*}{ G2 } & I: & \multicolumn{4}{|l|}{$\begin{array}{l}\frac{4(16.1 \%)}{70(46.4 \%)} \\
\end{array}$} \\
\hline & & II: & \\
\hline & & III & \multicolumn{4}{|l|}{$\begin{array}{l}68(45.0 \% \\
13(8.6 \%)\end{array}$} \\
\hline \multirow{2}{*}{ Bleeding } & G1 & & \multirow{2}{*}{\multicolumn{4}{|c|}{$\begin{array}{l}1880.4 \text { (SD 1225.7) } \\
1395.9 \text { (SD 1155.8) }\end{array}$}} \\
\hline & G2 & & & & & \\
\hline \multirow{2}{*}{ Surgical Time } & $\mathrm{G1}$ & & \multicolumn{4}{|c|}{364.0 (SD 148.7) } \\
\hline & $\mathrm{G} 2$ & & \multicolumn{4}{|c|}{310.1 (SD 174.2) } \\
\hline \multirow{4}{*}{$3 \mathrm{CO}$} & \multirow{2}{*}{ G1 } & & \multicolumn{4}{|l|}{$5(20.8 \%)$} \\
\hline & & $\mathrm{N}$ & $19(79.2 \%$ & & & \\
\hline & $G$ & & $25(16.6 \%$ & & & \\
\hline & GL & & $126(83.4$ & & & \\
\hline & & & & PreOp & $6 \mathrm{~m}$ & $2 y$ \\
\hline & & 0 & & 44.8 & 39.0 & 32.9 \\
\hline & & & 2 function & 3.02 & 3.00 & 3.28 \\
\hline & G1 & & 2 subtotal & 2.82 & 3.25 & 3.45 \\
\hline & & & & 33.5 & 35.5 & 38.5 \\
\hline HRQOL & & & MCS & 42.1 & 43.0 & 45.5 \\
\hline & & 0 & & 37.7 & 27.9 & 23.1 \\
\hline & & & 2 function & 3.15 & 3.32 & 3.70 \\
\hline & G2 & & 2 subtotal & 2.91 & 3.49 & 3.67 \\
\hline & & & & 36.4 & 40.5 & 43.8 \\
\hline & & & MCS & 43.9 & 48.1 & 47.3 \\
\hline & & & & PreOp & $6 \mathrm{~m}$ & $2 y$ \\
\hline & & & $\mathrm{rCobb}$ & 38.8 & 20.2 & 20.6 \\
\hline & & & tal Balance & 72.7 & 39.3 & 34.6 \\
\hline & G1 & $\mathrm{P}$ & & 54.3 & 54.1 & 55.1 \\
\hline & & $\mathrm{P}$ & & 23.8 & 20.7 & 21.4 \\
\hline Radiographic & & $s$ & & 30.4 & 33.4 & 33.6 \\
\hline & & & r Cobb & 41.3 & 28.3 & 20.8 \\
\hline & & & tal Balance & 21.8 & 4.1 & 12.2 \\
\hline & G2 & $\mathrm{PI}$ & & 52.3 & 52.0 & 51.5 \\
\hline & & $\mathrm{PT}$ & & 18.9 & 17.7 & 17.4 \\
\hline & & SS & & 33.3 & 34.2 & 34.1 \\
\hline
\end{tabular}

\section{QF11}

\section{IMPACT OF ILIAC INSTRUMENTATION ON THE QUALITY} OF LIFE OF PATIENTS WITH ADULT SPINE DEFORMITY

Mar Pérez Martín-Buitrago, Javier Pizones, Francisco Javier Sánchez Pérez-Grueso, Mariana Díaz-Almirón, Emre Acaroglu, Ibrahim Obeid, Frank Kleinstueck, Ahmet Alanay, Ferrán Pellisé, European Spine Study Group ESSG

\section{Hospital Universitario La Paz, Madrid, Spain}

Introduction: Surgical treatment of adult spine deformity has been shown to improve function and relieve pain. Some patients require pelvic fixation with iliac screws, and this may affect some of their daily activities and functions. The aim of the study was to analyze if iliac instrumentation affects the quality of life of the patients with adult spine deformity.

Methods: A retrospective cohort analysis of data collected prospectively in an adult deformity multicenter database was performed. Inclusion criteria were patients with a long arthrodesis of at least 8 levels and whose lowest instrumented vertebrae (LIV) were L5 or below. We analyzed age; Cobb angle; coronal and sagittal balance, number of levels instrumented; health-related quality of life questionnaires (HRQLq) ODI and COMI; and physical domains of SRS22 and SF-36 before surgery, at 6 months, and at 2 years' follow-up. Statistical analysis was performed with Student's $t$ test and MannWhitney U test.
Results: A total of 129 patients met the inclusion criteria. They were separated into 2 groups: "Iliac yes", with the LIV at the Ilium ( $N=104)$, and "iliac no," with the LIV at L5/S1/S2 $(\mathrm{N}=24)$. Prior to surgery, both groups were homogeneous for all variables except age and Cobb angle. The group "iliac yes" were older: $\mathrm{Me}=66$ years $(\mathrm{IQR}=59.25-71.00)$, while group "iliac no": $\mathrm{Me}=56$ years $(\mathrm{IQR}=46.00-69.50)(\mathrm{p}=0.008)$ and Cobb angle was larger in "iliac no": $\mathrm{Me}=45^{\circ}(\mathrm{IQR}=35.00-56.00)$, than in "iliac yes": $\mathrm{Me}=31.00^{\circ}(\mathrm{IQR}=18.00-48.00)(\mathrm{p}=0.019)$. The number of fused levels was larger in "iliac yes", $\mathrm{x}=12.6$ $(\mathrm{SD}=3.4)$, than in "iliac no," $\mathrm{x}=10.9(\mathrm{SD}=3.0)(\mathrm{t}=2.28$, $\mathrm{p}=0.020$ ). No statistically significant differences were found in the other analyzed parameters either at 6 months or at 2-years' follow up (see attached Table).

Conclusion: Both groups were fairly homogenous and comparable. Iliac instrumentation was more frequent in longer arthrodesis and older patients. The HRQLq scores were similar in both groups preoperatively and at follow up. Therefore, with the currently available tools, we cannot state that iliac instrumentation has a negative influence on patient's quality of life.

Disclosures: Author 1: grants/research support: DepuySynthes; Author 2: grants/research support: DePuy Synthes Johnson and Johnson, consultant: DePuy Synthes Johnson and Johnson; Author 3: grants/research support: DEPUY SINTHES, consultant: K2M. DEPUY SINTHES; Author 4: none; Author 5: grants/research support: Depuy Synthes, Medtronic, stock/shareholder: IncredX, royalties: Zimmer Biomet, AOSpine; Author 6: grants/research support: depuy synthes, consultant: depuy synthes, medtronic, royalties: alphatec, spineart clariance; Author 7: grants/research support: Depuy-Synthes; Author 8: grants/research support: DePuy, consultant: Medtronic; Author 9: grants/research support: dePuy Synthes Spine, Medtronic; Author 10: grants/research support: DePuy-synthes; Author 11: grants/research support: DePuy-Synthes.

\begin{tabular}{|c|c|c|c|c|c|c|c|}
\hline & & \multicolumn{3}{|c|}{ Before surgery } & \multicolumn{3}{|c|}{2 years } \\
\hline & & Median & \begin{tabular}{|l|l} 
Interquartile range \\
\end{tabular} & p & Median & $\begin{array}{l}\text { Interguartile } \\
\text { range }\end{array}$ & $\mathrm{p}$ \\
\hline \multirow{2}{*}{ Age } & \begin{tabular}{|l} 
Nliac YES \\
\end{tabular} & 66 años & de 59.25 a 71.00 & \multirow{2}{*}{$0.009^{*}$} & & & \\
\hline & Iliac No & 56 años & de 46.00 a 69.50 & & & & \\
\hline \multirow{2}{*}{ cobb } & \begin{tabular}{|l|l|} 
lliac YES \\
\end{tabular} & 31.009 & de 18.00 a 48.00 & \multirow{2}{*}{$0.019^{*}$} & 13.009 & de 7.25 a 22.5 & \multirow{2}{*}{0.255} \\
\hline & |lliac No & 45.009 & de 35.00 a 56.00 & & $19.00 \mathrm{e}$ & de 8.00 a 26.00 & \\
\hline \multirow{2}{*}{ св } & \begin{tabular}{|l} 
Hliac YES \\
\end{tabular} & $20.68 \mathrm{~mm}$ & $\begin{array}{ll}\text { de } 9.28 \text { a } 34.62 \\
\end{array}$ & \multirow{2}{*}{0.086} & $16.8 \mathrm{~mm}$ & $\begin{array}{ll}\text { de } 6.40 \text { a } 30.83 \\
\end{array}$ & \multirow{2}{*}{0.953} \\
\hline & |lliac No & $12.84 \mathrm{~mm}$ & de 5.59 a 28.68 & & $15.2 \mathrm{~mm}$ & de 6.00 a 31.25 & \\
\hline \multirow{2}{*}{ SVA } & \begin{tabular}{|l|l|} 
Nliac YES \\
\end{tabular} & $68.30 \mathrm{~mm}$ & de 23.47 a 110.37 & \multirow{2}{*}{0.244} & $37.94 \mathrm{~mm}$ & de 14.40 a 74.00 & \multirow{2}{*}{0.548} \\
\hline & |lliac No & $36.85 \mathrm{~mm}$ & de 0.00 a 88.58 & & $34.5 \mathrm{~mm}$ & de 10.70 a 66.09 & \\
\hline \multirow{2}{*}{ ODI } & \begin{tabular}{|l|l|l|l|l} 
lliac YES \\
\end{tabular} & 49.00 & de 38.00 a 64.00 & \multirow{2}{*}{0.958} & 40.00 & de 18.00 a 58.00 & \multirow{2}{*}{0.289} \\
\hline & |lliac No & 50.00 & de 41.00 a 61.00 & & 30.00 & de 20.00 a 49.00 & \\
\hline \multirow{2}{*}{$\begin{array}{l}\text { SRs22 } \\
\text { function }\end{array}$} & $\begin{array}{ll}\text { lliac YES } \\
\end{array}$ & 2.80 & de 2.30 a 3.00 & \multirow{2}{*}{0.414} & 3.00 & de 2.40 a 3.71 & \multirow{2}{*}{0.217} \\
\hline & |liac No & 2.45 & de 2.00 a 2.80 & & 3.40 & de 2.80 a 3.90 & \\
\hline \multirow{2}{*}{ SRS22 pain } & \begin{tabular}{|l} 
Hliac YES \\
\end{tabular} & 2.30 & de 1.78 a 3.00 & \multirow{2}{*}{0.277} & 3.30 & de 2.50 a 4.00 & \multirow{2}{*}{0.751} \\
\hline & |lliac No & 2.00 & de 1.65 a 2.50 & & 3.00 & de 2.12 a 4.10 & \\
\hline \multirow{2}{*}{ SRS22 } & \begin{tabular}{|l} 
lliac YES \\
\end{tabular} & 2.58 & de 2.05 a 3.00 & \multirow{2}{*}{0.974} & 3.11 & de 2.50 a 3.85 & \multirow{2}{*}{0.400} \\
\hline & |lliac No & 2.52 & de 2.03 a 2.95 & & 3.40 & de 2.70 a 3.75 & \\
\hline \multirow{2}{*}{ S36 PCS } & |lliac YES & 32.45 & de 27.83 a 37.87 & \multirow{2}{*}{0.655} & 37.48 & de 30.45 a 45.36 & \multirow{2}{*}{0.744} \\
\hline & |lliac No & 31.53 & de 25.52 a 37.88 & & 40.50 & de 30.93 a 46.65 & \\
\hline \multirow{2}{*}{ SF36 PF } & \begin{tabular}{|l|l|} 
Iliac YES \\
\end{tabular} & 30.75 & de 25.01 a 36.49 & & 36.49 & de 26.92 a 44.15 & \\
\hline & $\mid$ |liac No & 32.66 & de 27.39 a 40.32 & 0.385 & 42.23 & de 31.70 a 46.06 & 0.199 \\
\hline $5836 \mathrm{PP}$ & \begin{tabular}{|l|l|} 
Hliac YES \\
\end{tabular} & 30.21 & de 23.47 a 36.95 & م & 32.46 & de 30.21 a 44.24 & O1989 \\
\hline & Illac No & 27.96 & de 24.03 a 34.14 & 0.904 & 39.19 & de 30.21 a 49.30 & \\
\hline & \begin{tabular}{|l} 
lliac YES \\
\end{tabular} & 33.77 & de 30.55 a 38.21 & 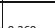 & 38.21 & de 30.55 a 46.68 & ברק \\
\hline SF36 BP & |lliac No & 30.55 & de 26.52 a 34.58 & & 38.21 & de 27.53 a 49.70 & \\
\hline & Hliac YES & 40.35 & de 33.22 a 48.43 & & 43.68 & de 37.61 a 53.19 & - \\
\hline $5 F 36 \mathrm{GH}$ & |liac No & 38.44 & de 33.22 a 47.48 & 0.990 & 43.68 & de 36.18 a 49.62 & 0.278 \\
\hline 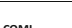 & \begin{tabular}{|l|l|} 
Hliac YES \\
\end{tabular} & 7.30 & de 5.90 a 8.40 & & 4.60 & de 2.23 a 7.10 & Dava \\
\hline & Iliac No & 7.00 & de 5.90 a 9.50 & 0.914 & 3.95 & de 2.97 a 7.10 & \\
\hline & \begin{tabular}{|l|l|} 
Iliac YES \\
\end{tabular} & & & & 12.6 & de 10.00 a 15.00 & \\
\hline № levels & Iliac No & & & & 10.9 & de 8.00 a 14.00 & $0.020^{*}$ \\
\hline
\end{tabular}




\section{QF12}

\section{PREDICTIVE MODELS TO AID SURGICAL DECISION-} MAKING FOR THORACOLUMBAR ADULT SCOLIOSIS

Javier Pizones, Mar Pérez Martín-Buitrago, Francisco Javier Sánchez Pérez-Grueso, Mariana Díaz-Almirón, Ahmet Alany, Ibrahim Obeid, Emre Acaroglu, Frank Kleinstueck, Ferrán Pellisé, European Spine Study Group ESSG

\section{Hospital universitario La Paz, Madrid, Spain}

Introduction: There is still a lack of information regarding the ideal moment when a thoracolumbar adult scoliosis patient should undergo surgery and who could obtain the highest clinical benefit with the least complications. The aim of the study was to analyze a multicenter cohort of thoracolumbar adult scoliosis operated patients, seeking information that may aid in surgical decision-making.

Methods: Retrospective analysis of prospectively collected data from an adult deformity multicenter database. Patients operated for thoracolumbar/lumbar (TL) curves were included. We selected as predictive variables for decision-making: age, coronal and sagittal deformity parameters, and clinical symptoms evaluated by ODI and SRS-22 questionnaires. As answer variables, we chose those that could quantify the risks (blood loss, surgical time, surgical aggressiveness, and complications) and benefits (2-year postoperative HRQoL questionnaires) of surgery. Multivariate stepwise regression analysis was performed to create predictive models.

Results: 175 patients met the inclusion criteria. Preoperative TLCobb, SVA, and ODI predicted blood loss $(\mathrm{R} 2=0.18)$. Preoperative TLCobb and SVA predicted surgical time $(\mathrm{R} 2=13.7)$. Lumbar mismatch predicted surgical aggressiveness $(\mathrm{R} 2=15.1)$. Preoperative pain predicted postoperative pain $(\mathrm{R} 2=25.1)$. Initial self-image, ODI, and preoperative function predicted final self-image perception $(\mathrm{R} 2=29.9)$. Preoperative age, TLCobb, and SVA predicted complications $(\mathrm{OR}=1)$.

Age increased the risk of postoperative complications but did not increase surgical aggressiveness or influenced in clinical results. The bigger the coronal curve and the sagittal misalignment, the greater was the surgical time and blood loss and the higher was the rate of complications, but without association with either surgical aggressiveness or clinical results. The higher the lumbar mismatch, the greater was the surgical aggressiveness, although neither blood loss nor complications increased necessarily. Preoperative clinical perception influenced postoperative clinical perception, independent of either surgical parameters or complications.

Conclusion: These predictive models may aid in TL scoliosis decision-making. Age increased the risk of complications; deformity parameters predicted surgical time, blood loss and rate of complications; lumbar mismatch was associated with surgical aggressiveness; and self-image and function predicted clinical 2-year outcomes.

Disclosures: Author 1: grants/research support: DePuy Synthes Johnson and Johnson, consultant: DePuy Synthes Johnson and Johnson; Author 2: grants/research support: DepuySynthes; Author 3: grants/research support: DEPUY SINTHES, consultant: K2M. DEPUY SINTHES; Author 4: none; Author 5: grants/research support: DePuy, consultant: Medtronic; Author 6: grants/research support: depuy synthes, consultant: deputy synthes, medtronic, royalties: alphatec spine, spineart, clariance; Author 7: grants/research support: Depuy Synthes, Medtronic, stock/shareholder: IncredX, royalties: Zimmer Biomet, AOSpine; Author 8: grants/research support: Depuy-Synthes; Author 9: grants/research support: DePuy Synthes Spine, Medtronic, consultant: DePuy Synthes Spine, Biomet; Author 10: grants/research support: DePuy-Synthes.

\section{QF13}

GLOBAL ALIGNMENT AND PROPORTION (GAP) SCORE: DEVELOPMENT AND VALIDATION OF A NEW METHOD OF ANALYZING SPINOPELVIC ALIGNMENT TO PREDICT MECHANICAL COMPLICATIONS AFTER ADULT SPINAL DEFORMITY SURGERY

Caglar Yilgor, Nuray Sogunmez, Yasemin Yavuz, Ibrahim Obeid, Frank S. Kleinstueck, Francisco Javier Sanchez Perez-Grueso, Emre R. Acaroglu, Ferran Pellisé, Ahmet Alanay, ESSG European Spine Study Group

School of Medicine, Acibadem University, Istanbul, Turkey

Background: The restoration of normal sagittal alignment is a critical goal in ASD surgery to achieve favorable outcomes and prevent mechanical complications. Schwab's sagittal modifiers have been accepted as targets for appropriate alignment but addressing these does not always prevent high mechanical complication/revision rates. This may be because the linear absolute numerical parameters do not cover the whole PI spectrum, and the distribution of lordosis, pelvic anteversion and negative malalignment are not considered as potential causes of failure.

Purpose: The aim was to develop and validate a PI-based proportional scoring system to better quantify the sagittal spinopelvic shape and alignment.

Methods: This study was a retrospective analysis of a prospectively collected multicentric data of ASD patients. 222 patients $(168 \mathrm{~F}$, $54 \mathrm{M}$ ) who had $\geq 4$ levels fusion with $\geq 2$ years follow up were included to this study. Mean age was $52.2 \pm 19.3$ (18-84) years. Mean follow up was $28.8 \pm 8.2$ (24-62) months. PJK/PJF, DJK/DJF, rod brekage and implant-related complications were recorded as mechanical complications. Revision surgeries performed due to mechanical complications were recorded as mechanical revisions. ODI, COMI, SF-36 PCS, MCS and SRS-22 pain, function, menthal health, self-image and subtotal scores were used as patient-reported Health Related Quality of Life (HRQoL) scores. GAP score was developed and validated in groups of patients randomly assigned to derivation $(\mathrm{n}=148,66.6 \%)$ and validation $(\mathrm{n}=74,33.3 \%)$ cohorts. GAP score parameters comprised Relative Pelvic Version [Measured minus Ideal Sacral Slope (SS)], Relative Lumbar Lordosis (Measured minus Ideal LL), Lordosis Distribution Index (L4-S1 lordosis/L1-S1 lordosisx100), Relative Spinopelvic Alignment (Measured minus Ideal Global Tilt) and age factor. The predictive accuracy of the GAP score was analyzed using ROC analyses. Associations between GAP categories and mechanical complications were analyzed using Cochran-Armitage tests. Differences in HRQoL scores between GAP categories were analyzed using one-way analysis of variance (ANOVA).

Results: In the validation cohort, 32 pts (43.2\%) experienced mechanical complications and 17(23.0\%) underwent mechanical revision. The area under curve for the GAP score in predicting mechanical complications was 0.92 (SE: $0.034, p<0.001,95 \% \mathrm{CI}$ 0.85-0.98). Postoperatively GAP proportioned patients had a mechanical complication rate of $6.1 \%$, while GAP moderately-disproportioned and severely-disproportioned patients had rates of 47.4 and $95.5 \%$, respectively.

Conclusion: The GAP Score denotes "normal" and "pathologic" spinal shape and alignment as a single score for every PI size. This PIbased proportional method of analyzing the sagittal plane predicts mechanical complications in ASD surgery. Setting surgical goals according to the GAP Score may decrease the incidence of mechanical complications. 
Disclosures: Author 1: none; Author 2: grants/research support: DePuy Synthes; Author 3: none; Author 4: grants/research support: DEPUY SYNTHES, consultant: DEPUY SYNTHES, MEDTRONIC, royalties: ALPHATEC, SPINEART, CLARIANCE; Author 5: grants/ research support: Depuy-Synthes; Author 6: grants/research support: DEPUY SINTHES, consultant: K2M. DEPUY SINTHES; Author 7: grants/research support: Depuy Synthes, Medtronic, stock/shareholder: IncredX, royalties: Zimmer Biomet, AOSpine; Author 8: grants/research support: DePuy Synthes Spine, Medtronic, consultant: DePuy Synthes Spine, Biomet; Author 9: grants/research support: Depuy Synthes, consultant: Medtronic; Author 10: grants/research support: DePuy-synthes.

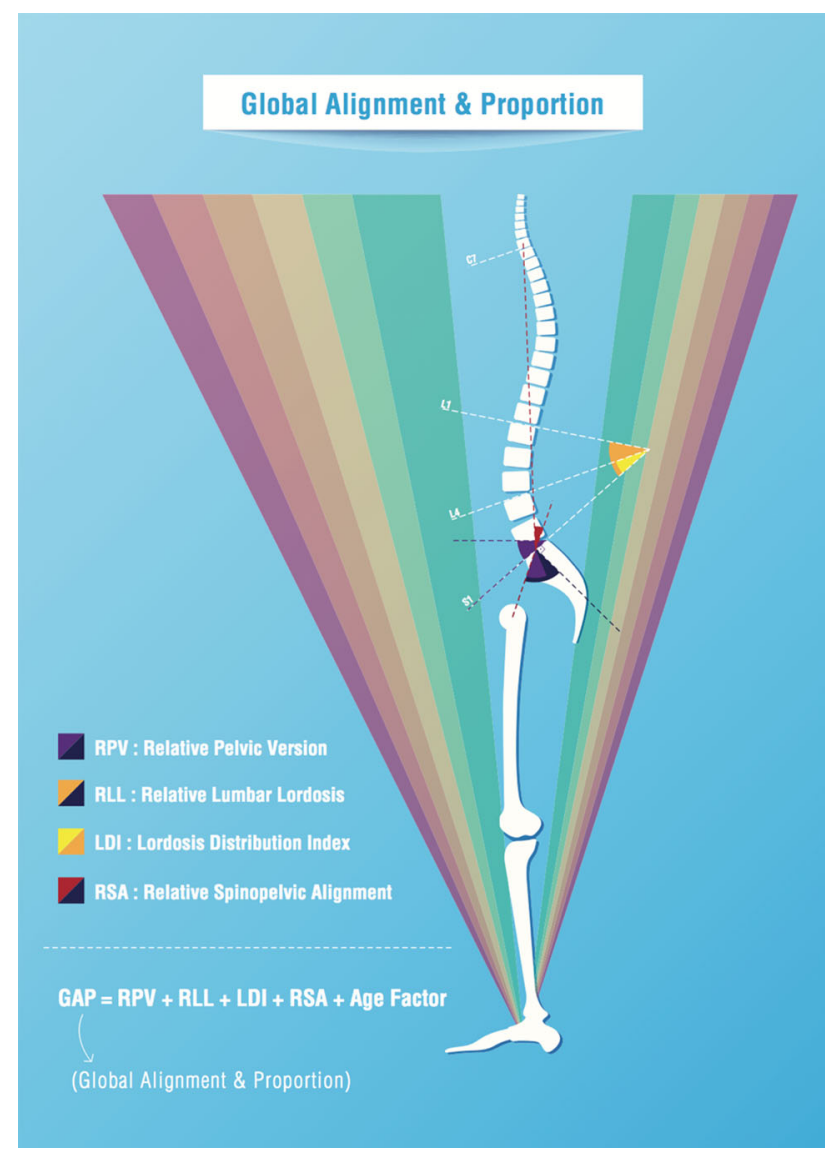

\section{QF14}

\section{RELATIVE PELVIC VERSION (RPV): A NEW PI-BASED PROPORTIONAL PARAMETER THAT QUANTIFIES PELVIC VERSION MORE PRECISELY COMPARED TO PT}

Caglar Yilgor, Nuray Sogunmez, Yasemin Yavuz, Ibrahim Obeid, Frank S. Kleinstueck, Francisco Javier Sanchez Perez-Grueso, Emre R. Acaroglu, Ferran Pellisé, Ahmet Alanay, ESSG European Spine Study Group

School of Medicine, Acibadem University, Istanbul, Turkey

Background: Quantification of the spinopelvic version with the simplistic criterion of PT $>20^{\circ}$ has limitations and needs to be adapted to the intrinsic pelvic morphology of each patient. PT $<20^{\circ}$ is accepted to improve outcomes and prevent mechanical complications. Increased PT is reported to indicate retroversion and disability. For a patient with large PI, PT $>20^{\circ}$ can be an anatomic trait and PT $<20^{\circ}$ may be disabling indicating that Schwab's criterion ignores anteversion as a potential cause of failure. Small PI pts may need even lower PT values. Relative PelviVersion (RPV) is described as 'Measured minus Ideal Sacral Slope (SS)' and indicates the pelvic orientation relative to the ideal SS in proportion to PI. (Ideal SS $=0.59 x \mathrm{PI}+9$ ) $\mathrm{RPV}<-15^{\circ}$ : severe retroversion, $-15^{\circ} \leq \mathrm{RPV}<-7^{\circ}$ : moderate retroversion, $-7^{\circ} \leq \mathrm{RPV} \leq 5^{\circ}$ : aligned and $\mathrm{RPV}>5^{\circ}$ : anteversion. Purpose: The aim was the comparison of the discrimination powers of PT and RPV.

Methods: This study was a retrospective analysis of a prospectively collected data of adult spinal deformity patients. Inclusion criteria were $\geq 4$ levels fusion, and $\geq 2$ years follow up. 222 patients (168 female, 54 male) met the inclusion criteria. Mean age was $52.2 \pm 19.3$ (18-84) years. Mean follow up was $28.8 \pm 8.2$ (24-62) months. ODI, COMI, SF-36 PCS, MCS and SRS-22 pain, function, mental health, self image and subtotal scores were used as patientreported Health Related Quality of Life (HRQoL) scores. PJK/PJF, $\mathrm{DJK} / \mathrm{DJF}$, rod breakage and implant related complications were recorded as mechanical complications. Correlations between PT, RPV, PI and HRQoL were analyzed using Pearson Correlation Coefficient. PI values and mechanical complication rates in RPV subgroups for each PT category were compared using one-way ANOVA, Independent Samples t and Chi squared tests.

Results: Changes in PT were affected by changes in PI $(r=0.613$, $\mathrm{p}<0.001)$ reducing its ability to quantify pelvic version for different PI values. RPV was not effected by PI $(r=-0.108, p>0.05)$ and quantified pelvic version for all PI values. When analyzed by RPV, each of PT ' 0 ', ' + ' and ' ++ ' categories were further divided into 2 or 3 distinct subgroups of patients having different PI values $(\mathrm{p}=0.000$, $\mathrm{p}=0.000$ and $\mathrm{p}=0.029$, respectively). RPV subgroups within the same PT category displayed different mechanical complication rates ( $\mathrm{p}=0.000, \mathrm{p}=0.020$ and $\mathrm{p}=0.019$, respectively) (Figure). RPV had better correlations to ODI, COMI, SF-36 PCS, MCS and all SRS22 subdomains when compared to PT ( $\mathrm{p}<0.05)$.

Conclusion: As a linear value, PT is insufficient in quantifying normoversion for the whole PI spectrum. PI-based proportional parameter of RPV offers a proportional quantification of ante-, normo- and retroversion for all PI sizes. RPV reveals that each Schwab PT group consists of inhomogeneous subgroup of patients with different mean PI values and mechanical complication rates. RPV predicts mechanical complications more precisely and has better correlations to HRQoL scores than PT.

Disclosures: Author 1: none; Author 2: grants/research support: DePuy Synthes; Author 3: none; Author 4: grants/research support: DEPUY SYNTHES, consultant: DEPUY SYNTHES, MEDTRONIC, royalties: ALPHATEC, SPINEART, CLARIANCE; Author 5: grants/ research support: Depuy-Synthes; Author 6: grants/research support: DEPUY sinthes, consultant: K2M. DEPUY SINTHES; Author 7: grants/research support: Depuy Synthes, Medtronic, stock/shareholder: IncredX, royalties: Zimmer Biomet, AOSpine; Author 8: grants/research support: DePuy Synthes Spine, Medtronic, consultant: DePuy Synthes Spine, Biomet; Author 9: grants/research support: Depuy Synthes, consultant: Medtronic; Author 10: grants/research support: DePuy-synthes. 


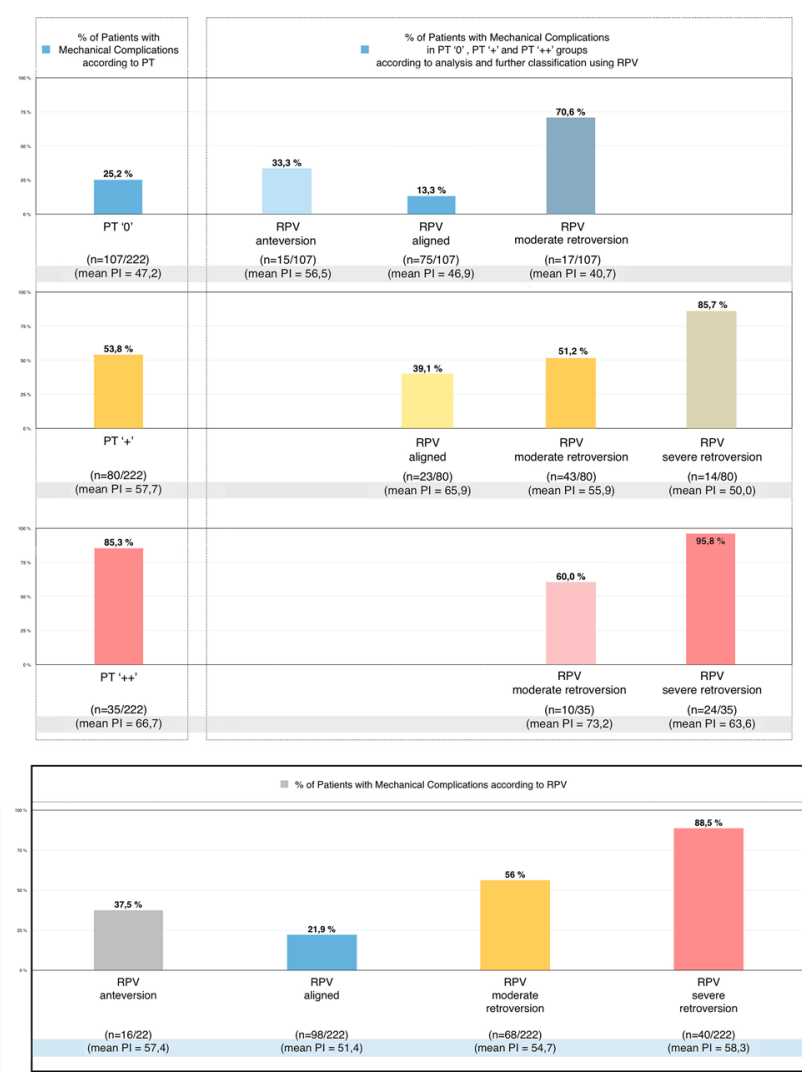

BASIC SCIENCE

\section{QF15 \\ COMPREHENSIVE BIOMECHANICAL ASSESSMENT OF THE HARDWARE BEHAVIOR AFTER PEDICLE SUBTRACTION OSTETOMY: FROM THEORY TO PRACTICE}

Andrea Luca, Alessio Lovi, Lisa Babbi, Gabriele Ristori, Sara Razza, Marco Brayda-Bruno, Fabio Galbusera

\section{IRCCS Galeazzi, Milan, Italy}

Introduction: The Pedicle Subtraction Osteotomy (PSO) is currently the gold standard for the correction of severe spinal imbalance: the osteotomy involves all three columns of the spine producing a massive concentration of stress on the hardware. The complication rate of the procedure and specifically the implant failure is substantially higher than in other corrective techniques. Aim of the study was to provide a comprehensive biomechanical model to investigate the behavior of different hardware constructs to understand and to overcome the criticalities of the procedure.

Materials: A finite element model of the L1-S1 spine region was developed and validated according to literature da-ta. The model was used as a base to simulate PSO at L3 and L4, both with and without spinal instrumentation. In particular, the following fixation configurations were investigated: (1) pedicle screw and rod fixation two levels above and below the osteotomy site; (2) posterior fixation + double rod construct at the osteotomy site; (3) intervertebral fixation with cages at the level below and (4) above the PSO as well as (5) both above and below the osteotomy. For configurations (1) and
(2), the effect of rod material and diameter was studied; rods with different diameters $(5-6 \mathrm{~mm})$ were simulated, as well as in Ti and CoCr alloys.

Results: The PSO without instrumentation determined a large destabilization of the spines in all directions (up to 58\% in axial rotation). Although all fixation configurations significantly reduced the iatrogenic instability, the peak stress values and the stress distribution were highly dependent on the simulated construct. Bi-lateral double rod constructs as well as intervertebral fixation with cages had the highest effect in reduc-ing the stress peaks in the rods (up to $37 \%$ for the double bilateral rods, $29 \%$ for the cage implanted in a single level and $53 \%$ for cages in both adjacent levels), which may be responsible of mechanical failure. No significant differences were found for the cages above or below the osteotomy, whereas the intervertebral support at both levels maximised the effect of intervertebral stabilization.

Discussion: Bilateral double $\mathrm{CoCr}$ rods constructs were effective in reducing the stresses acting on the rods, but by increasing the stiffness of the posterior construct induced a decrease of the load transmitted through the osteotomy site. On the contrary, the hardware implementation with interbody support reduced the rod stress by anteriorly shifting the load distribution, and may therefore have a double positive effect: to pro-tect the posterior construct and to facilitate the bone healing process at the osteotomy site.

On the basis of the current theoretical data we changed our surgical technique when performing a PSO in order to provide an adequate circumferential hardware support: a bilateral double $\mathrm{CoCr}$ rods construct with at least one interbody cage adjacent to the osteotomy site is nowadays routinely performed.

Disclosures: Author 1: none; Author 2: none; Author 3: none; Author 4: none; Author 5: none; Author 6: grants/research support: Medtronic-DepuySpine-K2M, consultant: Medtronic-DepuySpineK2M-Implanet-Spineguard; Author 7: none.

\section{QF16}

\section{THORACOLUMBAR ERECTOR SPINAE AND TRUNK OBLIQUES MUSCLE ACTIVITY DURING WALKING AT VARIOUS SPEEDS AND INCLINATIONS: DIFFERENCES BETWEEN YOUNG AND OLDER ASYMPTOMATIC ADULTS}

Rebecca Crawford, Leonardo Gizzi, Áine Ni Mhuiris, Angela Dieterich, Deborah Falla

${ }^{1}$ Institute for Health Sciences, Zurich University of Applied Sciences, Switzerland; ${ }^{2}$ Faculty of Health Professions, Curtin University, Australia; ${ }^{3}$ Continuum Biomechanics and Mechanobiology Research Group, University of Stuttgart, Germany; ${ }^{4}$ Institute for Neurorehabilitation Systems, University Medical Centre, Göttingen, Germany; ${ }^{5}$ Pain Clinic, Anesthesiology, University Medical Center Göttingen, Germany; ${ }^{6}$ Centre of Precision Rehabilitation for Spinal Pain (CPR Spine), School of Sport, Exercise and Rehabilitation Sciences, College of Life and Environmental Sciences, University of Birmingham, UK

Introduction: The power locus during walking shifts proximally from ankle to hip with age. However, surprisingly few studies measure thoracolumbar spinal muscle activity during walking despite differential activation within multifidus being shown between young and older adults during sagittal-plane motion. We examined whether this is also true for the thoracolumbar erector spinae (ES) and trunk obliques in asymptomatic adults during walking under various conditions. 
Methods: Ten volunteers in their 20s' (3f, $7 \mathrm{~m} ; 26.3 \pm 2.5$ years; BMI $23.5 \pm 4.5 \mathrm{~kg} / \mathrm{m} 2)$ and nine volunteers in their sixties-seventies [3f, $6 \mathrm{~m} ; 67.1 \pm 4.2$ years; BMI $25.0 \pm 2.9(\mathrm{p}=0.36)]$ walked on a treadmill in eight conditions; at $2 \mathrm{~km} / \mathrm{h}$ and $4 \mathrm{~km} / \mathrm{h}$, each at $0,1,5$, and $10 \%$ inclination. Surface EMG examined thoracolumbar ES [L3, thoracolumbar junction (TLJ), and thoracic kyphosis peak (TK)] and external (EO) and internal (IO) obliques. Activity for each muscle region was characterized by: the amplitude of the peak of activation (normalized to the maximal activity in the slow speed and $0 \%$ inclination), the position of the peak within the gait cycle $(0-100 \%)$, and the duration of muscle activity as a percent relative to the full gait cycle. Statistical analysis was undertaken using ANOVA.

Results: ES: Percentage of the gait cycle where peak ES amplitude occurred did not differ with group, speed or inclination. Peak activation of the L3 and TLJ ES was significantly greater for the older participants across all conditions $(\mathrm{p}<0.01)$ and was greater for both groups when walking at $4 \mathrm{~km} / \mathrm{h}$ at either 5 and $10 \%$ inclination compared to walking at $2 \mathrm{~km} / \mathrm{h}$ at 1,5 or $10 \%$ inclinations (all $\mathrm{p}<0.05$ ); TK ES did not differ. Duration of all ES activation relative to the full gait cycle was significantly longer for older than younger participants $(\mathrm{p}<0.05)$ but did not change with speed or inclination. Obliques: EO peak activation occurred earlier in the gait cycle for older participants $(\mathrm{p}<0.05)$ but did not differ with speed or inclination. EO was more active at $4 \mathrm{~km} / \mathrm{h}-10 \%$ compared to all at $2 \mathrm{~km} /$ $\mathrm{h}$; IO peak or amplitude did not differ far any condition. Duration of EO and IO activation was longer for older participants $(\mathrm{p}<0.001)$ and EO duration was longer when walking at $2 \mathrm{~km} / \mathrm{h}(\mathrm{p}<0.01)$.

Discussion: Differential activation of ES (at and below the TLJ) and obliques was shown between young and older adults under various walking conditions. Generally, older adults had higher peak activation and longer duration in EO and ES (L3 and TLJ) indicating altered muscle control with age. Speed of walking appeared more influential than inclination that may have implications for rehabilitation. Altered spinal muscle control with age may be linked e.g. to falls-history or to pain, which warrants further examination.

Disclosures: Author 1: none; Author 2: none; Author 3: none; Author 4: none; Author 5: none.

\section{QF17 \\ THE EFFECT OF UPPER INSTRUMENTED VERTEBRA SELECTION ON PROXIMAL JUNCTION LOADING}

Dominika Ignasiak, Tobias Peteler, Tamás Fekete, Daniel Haschtmann, Stephen J. Ferguson

Institute for Biomechanics, ETH Zurich, Zurich, Switzerland; Spine Center, Schulthess Clinic, Zurich, Switzerland

Proximal junctional kyphosis and failure (PJF) are estimated to affect up to $40 \%$ of adults who undergo long instrumented spinal fusion surgery [1]. The selection of the upper instrumented vertebra (UIV) has been postulated as a risk factor, but the findings of clinical studies are inconsistent. Since mechanical loads likely play a direct role in the development of the junctional disorders, insight into the loading conditions at the proximal segment after spinal fusion of various lengths would improve our understanding of the etiology of these disorders. Therefore, the objective of this work was to investigate the effect of UIV selection (for a fusion to the sacrum) on the loads acting on the proximal segment, using musculoskeletal modeling.

A previously developed and validated musculoskeletal rigid-body model of the thoracolumbar spine with articulated ribcage was used [2]. Spinal fusion was modeled by rigidly connecting fused vertebrae at a selected site. Inverse dynamics simulations of a full-range spine flexion were performed for fusions to the sacrum, with the upper instrumented vertebra at any level between L5 and T2.

Segmental flexion, bending moments on the passive spine structures (i.e., intervertebral discs and paraspinal ligaments) as well as compressive and shear forces were predicted for the proximal segment and compared to the corresponding estimates of the uninstrumented spine model. The model predicted an expected increase in segmental flexion at the proximal level, and a corresponding increase in passive moments for long fusions ( $\geq 6$ fused segments). The compressive forces were in general reduced, but the shear forces were substantially increased for fusions ending in the upper thorax ( $\geq 10$ fused segments).

In conclusion, a detailed biomechanical model of the instrumented thoracolumbar spine was established for the prediction of loads acting on the proximal junction. The combination of increased shear and bending moment at the proximal junction after long fusions may explain the observed mode of PJF, thus contribute to our understanding of the mechanical factors involved. In this preliminary study, a generic spinal posture was modeled and segmental motion was predicted, but the model can be adjusted to simulate subject-specific cases. Therefore, musculoskeletal modeling appears to be a promising and versatile method to support planning of spinal instrumentation surgeries in the future.

\section{References}

[1] Hart, et al. Neurosurgery Clinics of North America 24, 213-218, 2013.

[2] Ignasiak et al. J Biomech, 49(6), 959-966, 2016.

Acknowledgements: This work was supported by a grant from Mäxi Foundation, Switzerland.

Disclosures: Author 1: grants/research support: Mäxi Foundation, Switzerland; Author 2: employee: Synthes GmbH; Author 3: none; Author 4: none; Author 5: grants/research support: DePuy.
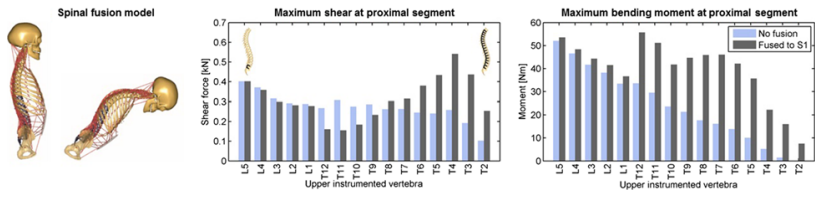

\section{QF18 \\ EFFECT OF GLUCOCORTICOID WITHDRAWAL ON GLUCOCORTICOID INDUCING BONE IMPAIRMENT}

Gengyang Shen, Hui Ren, De Liang, Ting Qiu, Zhida Zhang, Wenhua Zhao, Jinjing Huang, Xiang Yu

Department of Spinal Surgery, Guangzhou, China

Glucocorticoid (GC) withdrawal after a short-term use was common in clinical practice like immediate post-transplant period. However, previous studies without setting age-control group failed to determine whether the BMD recovery was sufficient and whether it is necessary to accept anti-osteoporosis therapy after GC withdrawal. The aim of this study was to investigate the effect of GC withdrawal on bone impairment in glucocorticoid-induced osteoporosis (GIOP) rats. Twenty-four female Sprague-Dawley rats (3 months' old) were randomly divided into two treatment groups: an untreated age-control group (Con $\mathrm{n}=12$ ); another group receiving a dexamethasone injection (DEXA $\mathrm{n}=12$ ). Animals in the Con group were euthanized at 3rd month (M3) and 6th month (M6), respectively. Six rats in the DEXA group were euthanized at 3rd month (M3), whereas GC intervention was withdrew in the remaining animals of DEXA group, 
which were euthanized at the end of 6th month (M6). Bone mass, bone microarchitecture, biomechanical properties of vertebrae, morphology, serum levels of PINP and $\beta$-CTX were evaluated. Compared with the Con(M3) group, the Con(M6) group showed significantly better bone quantity, morphology and quality. Compared with the Con(M3) group, the DEXA(M3) group showed significantly lower BMC, BMD, BS/TV, BV/TV, Tb.N, Tb.Th, vBMD, bone strength, compressive displacement, energy absorption capacity, PINP levels, $\beta$-CTX levels, and damaged trabecular morphology. And the same change trend was observed in the comparison between the Con(M6) group and DEXA(M6) group. Compared with the DEXA(M3) group, the DEXA(M6) group showed significantly higher BMC, BMD and AREA, but no significant difference in BS/TV, BV/TV, SMI, Tb.N, Tb.Th, Tb.Sp, vBMD, bone strength, bone stiffness, compressive displacement, energy absorption capacity, PINP levels, $\beta$-CTX levels, and improvement in trabecular morphology was observed. These results indicate that the reverse effect of GC withdrawal for 3 months on bone impairment in GIOP rats was insufficient, which implied that related anti-osteoporosis treatment might be still necessitated after GC withdrawal in clinical setting.

Disclosures: Author 1: none; Author 2: none; Author 3: none; Author 4: none; Author 5: none; Author 6: none; Author 7: none; Author 8: none.

\section{QF19 \\ MYRICITRIN INHIBITS RANKL-MEDIATED OSTEOCLASTOGENESIS BY ATTENUATING THE NF- B AND MAPKS SIGNALING PATHWAY}

Lingbo Kong, Xiaobing Yang

Honghui Hospital, Xi'an, China

Aim: Myricitrin is a natural flavonoid that inhibits nitric oxide (NO) transmission and has an atypical antipsychotic-like profile in animal models. The present study was performed to define effects of myricitrin on nuclear factor-kappaB ligand (RANKL)- stimulated osteoclast differentiation and the underlying mechanisms.

Methods: The bone marrow cells (BMMs) were harvested and induced with RANKL followed by treatment with myricitrin at several doses, and the differentiation of osteoclasts from these cells was evaluated by tartrate-resistant acid phosphatase (TRAP) staining and resorption pit formation assay. The effects of myricitrin on osteoclastogenesis were further studied by examining RANKL-induced osteoclast F-actin ring formation and osteoclast bone resorption. Moreover, we explored the mechanisms of these downregulation effects by performed Western blotting and quantitative RT-PCR examination. Results: Results demonstrated myricitrin strongly inhibited RANKL induced osteoclast formation when added during the early stage of BMMs cultures, suggesting that it acts on osteoclast precursors to inhibit RANKL/RANK signaling. Moreover, myricitrin markedly decreased the phosphorylation of p38, ERK, JNK, p65 and I- B degradation, and significantly suppressed c-Fos and nuclear factor of activated T-cells cytoplasmic 1 (NFATc1), both the key transcription factors during osteoclastogenesis. The results demonstrating myricitrin inhibits RANKL-mediated osteoclastogenesis by attenuating the NF- B and MAPKs signaling pathway. Conclusion: These results collectively suggested that myricitrin acted as an antiosteoclastogenesis agent by blocking osteoclast activation.

Disclosures: Author 1: none; Author 2: none.

\section{QF20 \\ CHRONIC INFECTION IN LUMBAR DISC HERNIATION: WHAT IS THE ROLE OF BIOFILM FORMATION?}

Søren Ohrt-Nissen, Blaine G Fritz, Jonas Walbom, Kasper N Kragh, Thomas Bjarnsholt, Benny Dahl, Claus Manniche

Department of Orthopaedics, Spine Unit, Rigshospitalet, Copenhagen, Denmark

Background/introduction: The potential relationship between lumbar disc herniation (LDH) and chronic bacterial infection has been widely debated. Propionibacterium acnes has emerged as the most likely microorganism and is known to cause chronic infection through the formation of biofilm aggregates; however biofilm has never been identified in LDH patients.

Purpose of the study: This study aimed to assess the bacteriology of LDH tissue, and to determine whether biofilm formation occurs.

Materials and methods: A prospective diagnostic study was carried out on patients with LDH undergoing primary discectomy. Control samples were obtained from patients operated for spinal fractures or deformities. Disc material was placed in RNA-later and formalin. Following DNA extraction, the presence of bacteria was determined using reverse transcription polymerase chain reaction (rtPCR). Sanger sequencing was performed for rtPCR positive samples. Formalinfixed tissue sections were embedded in paraffin, sectioned and stained by fluorescence in situ hybridization with peptide nucleic acid-binding probes (one $P$. acnes-specific probe and one universal bacterial probe). Confocal laser microscopy was used to examine the sections and visualize bacterial aggregates.

Results: A total of $52 \mathrm{LDH}$ patients and 15 controls were included. Bacterial DNA was detected in $16 / 52$ samples in the LDH group and $7 / 15$ in the control group $(p=0.404)$. Sequencing identified bacteria in 9/16 and 6/7 PCR-positive samples in the LDH and control groups, respectively (alignment $\geq 97 \%$ ). Only two LDH samples showed good alignments for $P$. acnes. Confocal microscopy demonstrated evidence of inflammation with a clear presence of polymorph nuclear leukocytes. Bacterial aggregates were observed in seven LDH patients, but was not confirmed by rtPCR and/or sequencing. No aggregates were observed in the control group.

Conclusion: Microscopy demonstrated bacterial aggregates in seven LDH patients indicating a chronic infection but the specific bacterial species of the aggregates could not be identified. Signs of a bacterial presence were found in $23 / 67$ samples by rtPCR, but with no significant difference between the LDH and control group. The discordance between rtPCR and microscopy may be due to the heterogeneous distribution and small size of biofilm aggregates.

Disclosures: Author 1: grants/research support: K2M; Author 2: none; Author 3: none; Author 4: none; Author 5: none; Author 6: grants/research support: Medtronic, K2M; Author 7: stock/shareholder: Persica Pharmaceutical ltd. 


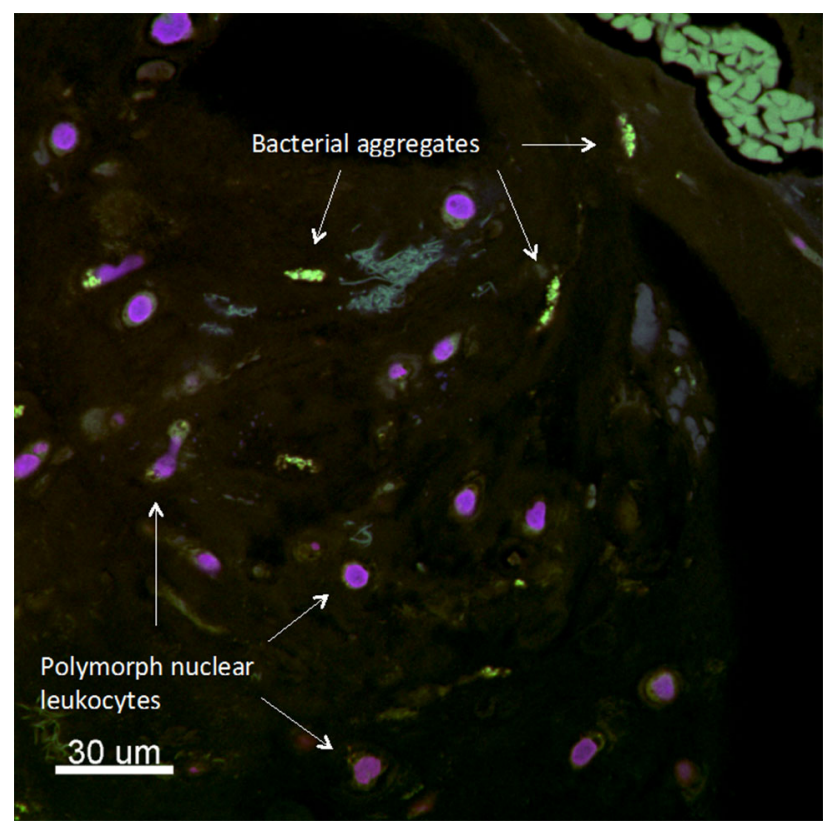

\section{QF21}

\section{ACETABULAR AND LOWER LIMB ALTERATIONS IN ADOLESCENT IDIOPATHIC SCOLIOSIS}

Mohammad Karam, Ayman Assi, Ziad Bakouny, Joe Ghanimeh, Georges Mjaess, Aya Karam, Chris Labaki, Fares Yared, Nour Khalil, Aren Joe Bizdikian, Wafa Skalli, Ismat Ghanem

${ }^{1}$ Laboratory of Biomechanics and Medical Imaging, Faculty of Medicine, University of Saint-Joseph, Beirut, Lebanon; ${ }^{2}$ Institut de Biomécanique Humaine Georges Charpak, Arts et Métiers, ParisTech, Paris, France

Introduction: Adolescent idiopathic scoliosis (AIS) is a deformity of the spine [1] which affects the morphology and orientation of the pelvis, leading to coronal pelvic obliquity [2] and changes in acetabular morphology [3, 4]. However, the effect of changes in pelvic orientation on acetabular cups and lower limbs has not been previously investigated in AIS. Our hypothesis is that acetabular and lower limbs morphology is altered in subjects with AIS.

Purpose: To describe acetabular and lower limb morphology in subjects with AIS.

Methods: 79 subjects with AIS (age $14.7 \pm 1.8$ years; 69F; Cobb $41^{\circ} \pm 15^{\circ}$ ) and 25 control subjects underwent full body biplanar X-rays. Spine, pelvis and lower limbs were reconstructed and their parameters calculated in 3D (Fig. 1a). Acetabular and lower limb parameters were evaluated bilaterally: center-edge-angle (CEA), acetabular orientation (anteversion, abduction, and tilt), Hip Knee Shaft angle (HKS), neck shaft angle, femoral anteversion, tibial torsion. The two sides of the pelvises in the frontal plane of each AIS subject were identified as either elevated (ES) or lowered (LS). Acetabular and lower limb parameters between the ES and LS of patients with AIS were compared (Wilcoxon's test). In order to explore the heterogeneous acetabular and lower limb alterations in subjects with AIS, the distribution of the morphological parameters of scoliotic patients were compared to those of control subjects based either on previously published thresholds [4] or the 1st quartile, median and 3rd quartile of the control sample (Fisher's exact test). Stepwise multinomial regression was computed in order to investigate determinants of acetabular and lower limb morphologies.
Results: The LS showed higher lateral coverage (CEA: $32^{\circ}$ vs $29^{\circ}$, $\mathrm{p}<0.001)$ and less abduction of the acetabulum $\left(55^{\circ}\right.$ vs $57^{\circ}$, $\mathrm{p}<0.001)$ compared to the ES in AIS. When both sides were compared to controls, the femoral head of the ES was shown to be less covered with a higher shift towards dysplasia; whereas the femoral head of the LS was shown to be over-covered [4] ( $p=0.001$, Fig. 1b). The acetabulum was shown to be more abducted in the ES when compared to the LS and controls (ES: $40 \%$ vs LS: $17 \%$, controls: $25 \%$, $\mathrm{p}=0.01)$. Both the ES and LS in AIS showed an increased prevalence of high HKS angle (ES: 47\%, LS: $61 \%$ vs controls: $25 \%, \mathrm{p}=0.003$ ). Overall, coronal pelvic obliquity, pelvic tilt and pelvic incidence $(\mathrm{p}<0.01)$ were the most significant determinants of differences in acetabular and lower limb morphology between AIS and controls.

Conclusion: The laterally tilted pelvis in AIS patients was found to produce an asymmetry in hip morphology between the ES and LS. The LS of the pelvis in AIS tends to present a less abducted acetabulum and an over-coverage of the femoral head by the acetabulum, which could predispose the hips to premature osteoarthritis.

\section{References}

1) James J 1954

2) Pasha $S 2014$

3) Legaye J 2011

4) Tannast M 2015

Disclosures: Author 1: none; Author 2: none; Author 3: none; Author 4: none; Author 5: none; Author 6: none; Author 7: none; Author 8: none; Author 9: none; Author 10: none.
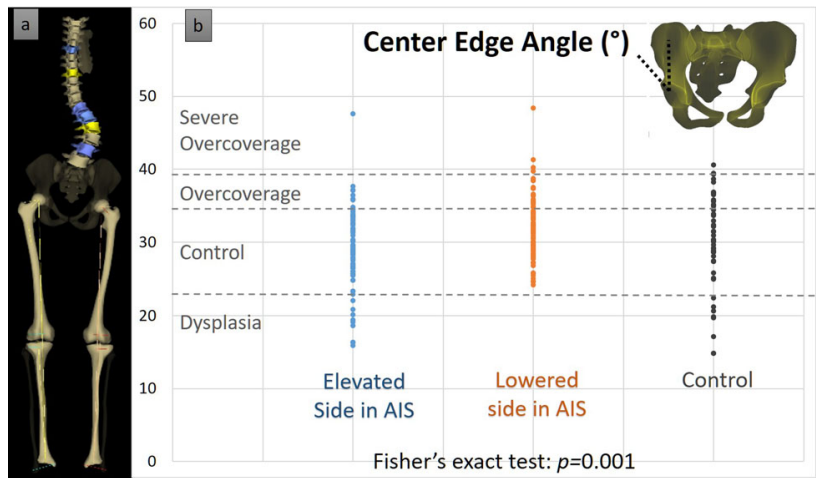

\section{QF22}

THE ALTERATION OF FULL BODY POSTURAL

\section{ALIGNMENT IN SUBJECTS WITH ADOLESCENT IDIOPATHIC SCOLIOSIS}

Nour Khalil, Ayman Assi, Aren Joe Bizdikian, Fares Yared, Ziad Bakouny, Wafa Skalli, Virginie Lafage, Gaby Kreichati, Ismat Ghanem, Khalil Kharrat

${ }^{1}$ Laboratory of Biomechanics and Medical Imaging, Faculty of Medicine, University of Saint-Joseph, Beirut, Lebanon; ${ }^{2}$ Institut de Biomécanique Humaine Georges Charpak, Arts et Métiers, ParisTech, Paris, France; ${ }^{3}$ Spine division, Hospital for Special Surgery, New York, USA

Introduction: Subjects with Adolescent Idiopathic Scoliosis (AIS) are known to have altered spino-pelvic alignment. However, there are no studies that have assessed the full body postural alignment in these subjects. Our hypothesis was that the alteration of postural alignment of AIS subjects extends beyond the spino-pelvic complex and includes the head, cervical spine and lower limbs. 
Purpose: To assess full body postural alignment of subjects with AIS. Materials and methods: 148 subjects with AIS [126F; age: $14.5 \pm 2$ years; Cobb: mean $\left.=37^{\circ}\left(13.5^{\circ}-99^{\circ}\right)\right]$ and 30 control subjects (22F) underwent full body biplanar X-rays. AIS subjects were divided into two groups: Major Thoracic (MT: $\mathrm{N}=72$ ) and Major Thoracolumbar/Lumbar curves (MTL: $\mathrm{N}=76$ ). Pelvic parameters (PI, SS, PT, coronal pelvic obliquity POb), global alignment parameters (C7-S1 SVA, Spino-Sacral Angle SSA, T1 and T9 tilt, CAM-HA: distance from the center of the auditory meatus plumbline to the hip axis), thoracic kyphosis TK, lumbar lordosis LL, knee flexion/extension, pelvic shift and cervical spine parameters $(\mathrm{C} 1-\mathrm{C} 2, \mathrm{C} 2-\mathrm{C} 7, \mathrm{~T} 1 \mathrm{Slope} \mathrm{T} 1 \mathrm{~s}, \mathrm{~T} 1 \mathrm{~s}-\mathrm{CL}$, Chin Brow Vertical Angle CBVA and cervical Sagittal Vertical Axis cSVA) were measured. Differences between the control group and the two AIS groups were evaluated using the Kruskal-Wallis test.

Results: Global sagittal alignment, CBVA and lower limb parameters were similar among the three groups ( $\mathrm{p}>0.05)$. TK (C:34, MT:24, MTL:29 ${ }^{\circ}$ and C7-CSL (C:5, MT:10, MTL:13 mm) were significantly different between all groups $(\mathrm{p}<0.001)$. In the MT group, PI and PILL were found to be significantly more elevated when compared to controls, $\left(53^{\circ}\right.$ vs $45^{\circ}, \mathrm{p}=0.02 ;-5^{\circ} \mathrm{vs}-11^{\circ}, \mathrm{p}=0.024$ resp.) as well as cSVA ( 9 vs $8 \mathrm{~mm}, \mathrm{p}=0.008$ ). However, T1 Slope and T1-CL were significantly decreased in the MT group when compared to the controls $\left(17^{\circ}\right.$ vs $22^{\circ}, \mathrm{p}=0.004 ; 9^{\circ}$ vs $\left.21^{\circ}, \mathrm{p}=0.016\right)$.

In the MTL group, POb was found to be significantly more elevated compared to controls (6 vs $4 \mathrm{~mm}, \mathrm{p}=0.017$ ) (Fig. 1).

Conclusion: This is the first study to analyze the full body alignment in subjects with different types of scoliotic curvatures. While horizontal gaze, global sagittal alignment and lower limb parameters were similar among the three groups, differences were found in coronal global alignment (POb and C7-CSL) and sagittal segmental parameters. All AIS subjects showed increased coronal global malalignment. The MT group presented a higher pelvic incidence and a thoracic hypokyphosis which lead to a compensatory increase in PI-LL to maintain their sagittal global alignment. Similarly, these subjects showed a decrease in the mismatch between $\mathrm{T} 1$ slope and cervical lordosis to maintain horizontal gaze. A less pronounced decrease in kyphosis was found in the MTL group, which allowed these subjects to maintain their sagittal global alignment and horizontal gaze without compensatory mechanisms.

Disclosures: Author 1: none; Author 2: none; Author 3: none; Author 4: none; Author 5: none; Author 6: none; Author 7: grants/research support: SRS, Stryker, DePuy Spine, K2M, NuVasive, consultant: NuVasive, stock/shareholder: Namaris INC, other financial report: DePuy Spine, NuVasive, K2M, MSD (speaking/teaching arrangements); Author 8: none; Author 9: none; Author 10: none.

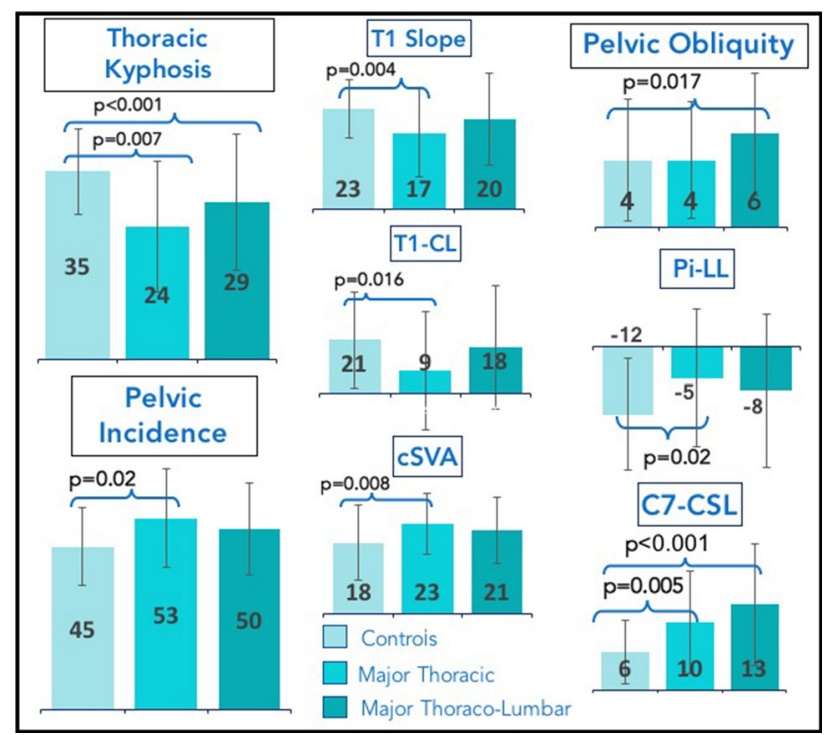

\section{QF23}

EFFECT OF GLOBAL POSTURAL ALIGNMENT ON GAIT: A STUDY ON 145 ASYMPTOMATIC SUBJECTS WITH A LARGE AGE RANGE

Joeffroy Otayek, Fares Yared, Aren Joe Bizdikian, Ayman Assi, Ziad Bakouny, Nour Khalil, Joe Ghanimeh, Chris Labaki, Abir Massaad, Virginie Lafage, Wafa Skalli, Ismat Ghanem, Khalil Kharrat, Gaby Kreichati

${ }^{1}$ Laboratory of Biomechanics and Medical Imaging, Faculty of Medicine, University of Saint-Joseph, Beirut, Lebanon; ${ }^{2}$ Institut de Biomécanique Humaine Georges Charpak, Arts et Métiers, ParisTech, Paris, France; ${ }^{3}$ Spine division, Hospital for Special Surgery, New York, USA

Introduction: Global postural alignment parameters have been shown to be major determinants of quality of life [1]. Gait is an essential task of functionality during daily living activities. It is still unknown how global postural alignment can influence gait. Our hypothesis was that global postural alignment has an impact on gait patterns.

Purpose: To investigate how global postural alignment can influence gait in asymptomatic subjects.

Methods: 145 asymptomatic subjects [age: $29.2 \pm 11$ years (18-59), 70F] underwent 3D gait analysis followed by full body biplanar X-rays. Kinematics of lower limb segments (pelvis, hip, knee, ankle and foot) were extracted in the 3 planes during the gait cycle. Full body $3 \mathrm{D}$ reconstructions were obtained from the biplanar X-rays and global postural alignment was evaluated by calculating: sagittal vertical axis (SVA), center of the auditory meatus plumbline to the hip axis, thoracic kyphosis (TK), lumbar lordosis (LL), pelvic parameters (PI, PT, SS). Agglomerative hierarchical clustering (AHC) was performed on each postural parameter to classify subjects into homogeneous groups. Differences in gait parameters between groups were evaluated using ANCOVA while adjusting for demographic confounding factors (age, weight, height and sex).

Results: The most discriminant postural parameter in gait kinematics was the SVA. AHC differentiated 3 groups of SVA: Gr1: $<-16 \mathrm{~mm}$, $\mathrm{N}=28$; Gr2: $(-16,10) \mathrm{mm}, \mathrm{N}=62$; and Gr3: $>10 \mathrm{~mm}, \mathrm{~N}=55$. Weight, height and sex were statistically different between groups (Gr1 vs Gr3: $68 \pm 16$ vs $77 \pm 13 \mathrm{~kg}, 167 \pm 10$ vs $173 \pm 10 \mathrm{~cm}$, $39 \mathrm{~F} / 23 \mathrm{M}$ vs $6 \mathrm{~F} / 22 \mathrm{M} ; \mathrm{p}<0.05)$. After correcting for confounding factors, Gr3 had a larger hip external rotation during gait $\left(15^{\circ} \mathrm{vs} 13^{\circ}\right.$ in Gr1), higher knee flexion during loading response at the beginning of the gait cycle ( 18 vs $14^{\circ}$ in Gr1), a delayed knee extension instant during the stance phase (39 vs $35 \%$ of gait cycle in Gr1), a smaller external foot progression angle ( $7^{\circ}$ vs $10^{\circ}$ in Gr1), and a larger step length (68 vs $65 \mathrm{~cm}$ in Gr1) (Fig. 1).

Conclusion: This is the first study demonstrating the influence of postural alignment parameters on gait. While inter-individual variations of TK and LL were not shown to impact gait, subjects with elevated SVA had their general gait modified even after correcting for confounding factors: higher duration with larger knee flexion during shock absorption phase at the beginning of gait cycle. While these results were shown in asymptomatic subjects with high SVA $(>10 \mathrm{~cm})$, higher SVA in subjects with spinal deformities could be a cause for more altered gait kinematics.

[1] Schwab F, Spine, 2012

Disclosures: Author 1: none; Author 2: none; Author 3: none; Author 4: none; Author 5: none; Author 6: none; Author 7: none; Author 8: none; Author 9: none; Author 10: grants/research support: SRS, Stryker, DePuy Spine, K2M, NuVasive, consultant: NuVasive, stock/ shareholder: Namaris INC, other financial report: DePuy Spine, NuVasive, K2M, MSD (speaking/teaching arrangements). 


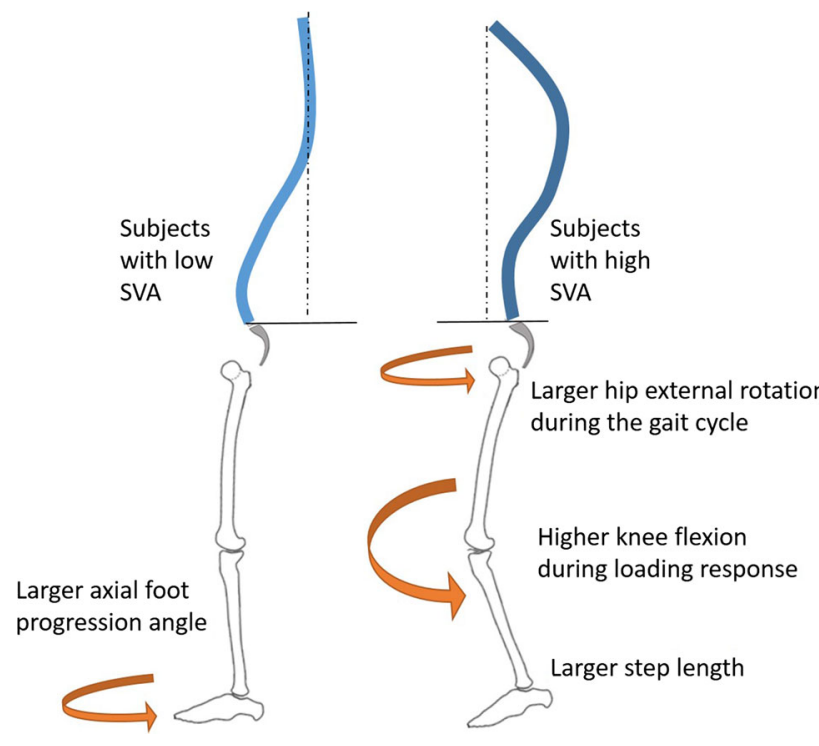

QF24

\section{THE IMPACT OF LOW BACK PAIN ON HEALTH-RELATED QUALITY OF LIFE IN SENIORS LIVING IN THE COMMUNITY}

Christine Cedraschi, Christophe Luthy, Anne-Françoise Allaz, François Herrmann, Catherine Ludwig

Division of General Medical Rehabilitation, Geneva University Hospitals and University of Geneva, Switzerland

Purpose: The present study aims at investigating the effects of low back pain (LBP) i.e., type of symptoms, activity limitations, frequency, duration, and severity on health-related quality of life (HRQoL) in a sample of 707 community-dwelling men and women aged $\geq 65$ years living in Switzerland.

Methods: The study is part of a larger survey conducted on a sample of older adults selected randomly from population records, stratified by age and sex. The Standardized Back Pain Definition was used to investigate LBP, and HRQoL was assessed by means of the EQ-5D, including Health Utility Index (HUI) measures. Data from the Standardized Back Pain Definition and from the EQ-5D were analyzed using descriptive statistics. Subsequently, inferential statistics were conducted to estimate the effect of LBP on HRQoL by means of regressions, including each of the HRQoL score as an outcome, and each of the LBP measures as a predictor. Age group, sex, linguistic area, and education were included in the models. Logit binary regression models were used for binary outcomes (Mobility, Self-Care, Usual-Activities, Anxiety/Depression, Pain/Discomfort), and linear regression models were used for linear outcomes (NRS and HUI).

Results: For more than half of the sufferers, pain was chronic, occurred most days or every day and induced activity limitations. One-third of the sufferers reported sciatica symptoms.

Individuals reporting every day pain $[\mathrm{B}=-13.54,95 \% \mathrm{CI}(-16.22$ to 10.87$)]$, severe pain [B $=-12.76,95 \% \mathrm{CI}(-15.77$ to 9.75$)]$ and 3 years since the last episode without pain $[\mathrm{B}=-8.99,95 \%$ $\mathrm{CI}=(-12.08$ to 9.91$)]$ lost nearly 10 points (i.e., $10 \%)$ of HRQoL. Sciatica affected HRQoL with individuals reporting pain running down the leg $[\mathrm{B}=-5.32,95 \% \mathrm{CI}(-7.94$ to 2.70$)]$, and running down the knee $[\mathrm{B}=-5.32,95 \% \quad \mathrm{CI}=-8.52$ to 2.51$)]$ demonstrating a reduction of HUI score of about $5 \%$ as compared to individuals not reporting symptoms of sciatica. Overall, the presence of pain running down the leg and/or down the knee, a high pain frequency, chronic pain, and usual pain intensity above the mean were strongly associated with a decrease of HRQoL. Since we controlled for the effect of sociodemographic variables, the results ascertain that individuals with LBP experienced a high burden of disease, especially when pain is running down the leg and/or down the knee.

Conclusion: These results provide further insight into the impact of qualitative aspects of LBP and in particular the importance of radiating leg pain and pain frequency and duration. While LBP related activity limitations had little impact on both self-rated overall health and Health Utility Index, radiating leg pain and pain frequency and duration were associated with significantly decreased scores on both dimensions. This study also confirms that LBP may be part of the definition of a subgroup of elderly at risk, especially in the presence of radiating leg pain and when LBP characteristics include high frequency and chronicity.

Disclosures: Author 1: none; Author 2: none; Author 3: none; Author 4: none; Author 5: none.

\section{QF25 \\ THE ASSOCIATED FACTORS OF LOW BACK PAIN IN PATIENTS WITH RHEUMATOID ARTHRITIS}

Kazuto Miura, Osamu Morita, Toru Hirano, Kei Watanabe, Naoto Endo, Yugo Shobugawa

Department of Spine and Spinal Cord Surgery, Niigata University, School of Medicine, Nagaoka, Japan

Background/introduction: The etiology of low back pain (LBP) in patients with rheumatoid arthritis (RA) is controversial.

Some reports indicate that the associated factors of LBP in patients with RA is radiological findings, and others demonstrate the association between LBP and disease activity of RA.

Purpose of this study: To elucidate the associated factors of LBP in patients with RA.

Materials and methods: 827 patients (168 men, 659 women, range, 21-91 years) with RA were enrolled in this cross-sectional study. Patients with previous spine surgery and/or bilateral total hip arthroplasty were excluded.

Clinical data included age, sex, onset age of RA, disease duration, body mass index (BMI), rheumatoid factor $(\mathrm{RF}), \mathrm{C}$-reactive protein (CRP), matrix metalloproteinase-3 (MMP-3), disease activity score in 28 joints-CRP (DAS28-CRP), medical history (ever or current use of corticosteroid, methotrexate and biological agents), surgical history of joints and visual analogue scale (VAS) scores for LBP were checked on clinical records.

Coronal and sagittal key parameters of spinopelvic alignment [Cobb angle, coronal shift of C7 plumb line (C7PL), PT, PI-LL, SVA, TPA] were measured by spinopelvic radiograph in standing position and the presence of vertebral fracture and spondylolisthesis were calculated. Dual X-ray absorptiometry of hip joint was also used to evaluate bone mineral density (BMD).

The patients with VAS $\geq 30$ were defined as moderate LBP and those with VAS $\geq 50$ were defined as severe LBP.

Univariate and multivariate logistic regression analyses were performed to determine the independent associated factors of moderate and severe LBP.

Results: The prevalence of moderate and severe LBP in patients with RA was $21.9 \%(181 / 827)$ and $11.1 \%$ (92/827).

According to multivariate analyses, the associated factors of moderate LBP were history of corticosteroid use $(\mathrm{P}=0.025$, OR 1.555 and 
95\% CI 1.056-2.289), presence of spondylolisthesis $(\mathrm{P}=0.036, \mathrm{OR}$ 1.602 and $95 \%$ CI 1.030-2.492), coronal shift of C7PL $(\mathrm{P}=0.035$, OR 1.020 and $95 \% \mathrm{CI} 1.001-1.040)$ and TPA $(\mathrm{P}<0.001$, OR 1.050 and $95 \%$ CI 1.031-1.070).

The associated factors of severe LBP were MMP-3 $(\mathrm{P}<0.001$, OR 1.002 and $95 \% \mathrm{CI} 1.000-1.005)$ and TPA $(\mathrm{P}=0.043$, OR 1.069 and 95\% CI, 1.044-1.095), respectively.

Conclusion: In this study, the associated factors of LBP in patients with RA were not only radiological findings but also the history of corticosteroid use and MMP-3 that suggests synovial joint damage and disc degeneration.

Disclosures: Author 1: none; Author 2: none; Author 3: none; Author 4: none; Author 5: none; Author 6: none.

\section{QF26 \\ MECHANISM OF NEURAL COMPLICATION INDUCED BY CORRECTIVE SURGERY FOR SPINAL DEFORMITY - MULTI-INSTITUTIONAL SURVEY BY THE SPINAL CORD MONITORING WORKING GROUP OF THE JAPANESE SOCIETY FOR SPINE SURGERY AND RELATED RESEARCH}

Kei Yamada, Yukihiro Matsuyama, Shigenori Kawabata, Muneharu Ando, Naoya Yamamoto, Sho Kobayashi, Shiro Imagama, Tsukasa Kanchiku, Kanichiro Wada, Nobuaki Tadokoro

Department of Orthopaedic Surgery, Kurume University, Kurume, Japan

Purpose: Spinal cord monitoring using transcranial electrical stimulation muscle evoked potential (TES-MEP) has been widely used to avoid iatrogenic spinal cord injury in medical institutions in Japan. The Spinal Cord Monitoring Working Group has reported that a loss of control wave over $70 \%$ amplitude in TES-MEP is suitable alarm criteria for spinal deformity surgery. However, a mechanism of insult to the spinal cord or spinal nerve root has not been considered. Therefore, the aim of this study was to investigate how corrective procedure cause insult to the spinal cord or spinal nerve root during spinal deformity surgery.

Methods: This is a prospective observational study with 631 patients with spinal deformity treated by spinal corrective surgery in fifteen medical institutions between 2010 and 2014. There are 288 patients with idiopathic scoliosis (IS), 244 with adult spinal deformity (ASD), 73 with syndromic scoliosis (SS) and 27 with congenital scoliosis (CS). All patients were performed spinal cord monitoring using TESMEP under same anesthesia conditions and spinal cord monitoring stimulation conditions. The alarm criteria were defined as $70 \%$ amplitude loss of TES-MEP to the baseline. The Authors investigated incidence of alert of TES-MEP, intervention after the alert during surgery and postoperative newly developed paralysis.

Results: 25 IS patients, 28 ASD patients, 11 SS patients and two CS patients had an alert during surgery. 8 true positive(TP) results, 2 false negative(FN), and 23 false positive (FP) were present among those patients with intraoperative alert. 35 patients showed wave recovery with or without intraoperative intervention, and did not have postoperative paralysis.

Five ASD patients and one CS had showed transient paralysis in spite of decompression or without any intervention after corrective procedure. One ASD patients with post-tuberculous spondylitis kyphosis had persisted paralysis after abscission of six thoracic nerve roots during vertebral column resection. One CS patient sustained persisted paralysis after dekyphosis using pedicle subtraction osteotomy. These
TP cases showed complete loss of TES-MEP amplitude without any wave recovery in the end of surgery. FN results included one with ASD and the other with CS. These two patients had transient paralysis without any alert during surgery. There were 9 FP cases in IS, 10 in ASD, 4 in SS.

Conclusion: IS patient have elastic spinal curve without spinal stenosis, spinal cord could have reversible ischemia by surgical corrective procedure. However, in ASD, SS and CS patient have relatively severe spinal deformity with spinal stenosis. Corrective procedure might have induced spinal nerve compression or nerve root over-traction. The nerve root traction could cause transient paralysis. Persisted paralysis could have induced by irreversible spinal cord ischemia through the abscission of thoracic nerve roots or 3-column osteotomy in ASD and CS.

Disclosures: Author 1: none; Author 2: none; Author 3: none; Author 4: none; Author 5: none; Author 6: none; Author 7: none; Author 8: none; Author 9: none; Author 10: none.

\section{QF27 \\ ISOLATED LUMBAR EXTENSION RESISTANCE TRAINING FOR TREATING CHRONIC LOWER BACK PAIN: A RETROSPECTIVE STUDY ON 626 PATIENTS}

Christoph Spang, Florestan Wagenblast, Desirée Haas, Florian Alfen

Department of Integrative Medical Biology, Umea, Sweden; Orthopaedic Spine Center, Würzburg, Germany; Institute of Occupational and Social Medicine and Health Services Research, Tübingen, Germany

Introduction: Chronic lower back pain is the most common cause for work disability worldwide and treatment is often difficult. It is known that neuromuscular deficits are involved and that specific and isolated lumbar extension resistance training can distinctly improve this condition (Steele et al. 2015). However, most studies have investigated the outcome on relatively small sample size. In the present study we have therefore analyzed the outcome of isolated resistance training on large sample size.

Methods: In a retrospective study the clinical outcome of isolated lumbar extension resistance training program on 626 consecutive patients with diagnosed chronic lower back pain (222 women, 404 men; mean age 50 years, range 18-78 years) was analyzed. All patients included had undergone 18-25 exercise sessions around two times per week. Scores containing information on pain perception and psychological features had been taken before and after the treatment. Furthermore, information about activity levels and pain duration had been noted before the therapy. Lumbar extension strength had been measured via a static strength test at the beginning of the first and last session. These measured strength levels had been compared to a data set collected from healthy individuals.

Results: At the first session of therapy lumbar extension strength deficits compared to healthy individuals were on average $45.49 \%$ (SD $20.62 \%$ ). After completing the treatment the deficit was on average $18.31 \%$ (SD 17.14\%). The strength levels significantly increased during the therapy (mean 27.08\%, SD 20.27) $(\mathrm{p}<0.01$ ). The vast majority of patients $(n=597,95.4 \%)$ kept or improved their strength levels. Furthermore, 554 patients $(88.5 \%)$ reported pain relief or at least maintenance. The pain symptoms improved in average $17.1 \%$ $(p<0.01)$. The effectiveness of the treatment correlated with the level of symptoms at the beginning $(\mathrm{p}<0.01)$ and also with the total increase of strength levels during the therapy $(p<0.01)$. The duration of pain and the activity levels had no influence on the outcome. 
Discussion: The results of this study show that isolated lumbar extension resistance training leads to a marked reduction of symptoms in the majority of patients with chronic lower back pain. The symptom levels before the treatment and the total achieved increase of strength during the therapy are indicators for the clinical outcome.

References

Steele J, Bruce-Low S, Smith D. (2015). PM R. 7, 169-187.

Disclosures: Author 1: none; Author 2: none; Author 3: none; Author 4: none.

\section{DEGENERATIVE SPINE}

\section{QF28 \\ LONG-TERM OUTCOMES OF LUMBAR TOTAL DISC REPLACEMENT VERSUS SPINAL FUSION FOR THE TREATMENT OF DDD: A NOVEL META-ANALYSIS OF RANDOMIZED CONTROLLED TRIALS}

\author{
Jack Zigler, Matthew Gornet, Nicole Ferko, Chris Cameron, \\ Francine Schranck, Leena Patel
}

Texas Back Institute, Center for Disc Replacement; Plano, TX, USA

Background context: To-date several meta-analyses of randomized trials have reported on the outcomes of lumbar disc replacement vs fusion, two surgical treatments for chronic low back pain following failure of conservative therapies. While several randomized trials have reported their 5-year outcomes, the results have yet to be pooled to assess the composite effectiveness of lumbar disc replacement and lumbar fusion as a whole.

Purpose: To evaluate the 5 year safety and efficacy outcomes of lumbar TDR compared with fusion.

Study design: Meta-analysis of randomized controlled trials.

Patient sample: Adults with discogenic low back pain due to singlelevel DDD who have failed conservative treatment previously reported in published studies.

Outcome measures: Owestry Disability Index (ODI) success, back pain scores (i.e., Visual Analog Scale (VAS) or Numeric Rating Scale), patient satisfaction, and reoperations defined as device-related failures resulting in subsequent surgical intervention of reoperation, revision, removal or supplemental fixation.

Methods: Selection criteria: RCTs comparing the treatment effects of lumbar TDR with fusion at 5 years in lumbar DDD patients. Databases searched included PubMed/MEDLINE and CENTRAL between the years of 2000 and 2015. Meta-analyses were conducted using a random-effects model; analyses were reported as relative risk ratios (RR) and mean differences (MD). Sensitivity analyses were conducted for different outcome definitions, high loss to follow-up and high heterogeneity.

Results: The literature search yielded a total of 2429 citations, with 180 full-text articles retrieved for eligibility assessment. After fulltext review, a total of 4 studies with 1325 patients were included in the meta-analysis. Pooled analysis demonstrated that lumbar TDR patients had a significantly greater likelihood of ODI success (RR 1.09; 95\% CI 1.00, 1.19; $\mathrm{p}=0.05$ ) and patient satisfaction (RR 1.13; $95 \%$ CI $1.03,1.24 ; p=0.009)$ than patients having fusion. Furthermore, lumbar TDR patients demonstrated a significantly lower risk of reoperation (RR $0.52 ; 95 \%$ CI $0.35,0.77 ; p=0.001$ ) than those with fusion. While not statistically significant, total disc replacement had better improvement in back pain score than fusion (MD $-2.79,95 \%$ $\mathrm{CI}-8.09,2.51 ; \mathrm{p}=0.30)$. Heterogeneity was reported to be low in this meta-analysis i2 ranging from 0 to $39 \%$. A sensitivity analysis was completed on all results. Results for ODI success and patient satisfaction were sensitive to different outcome definitions, but remained in favor of TDR.

Conclusions: The long-term analysis of the safety and efficacy profile of lumbar TDR vs fusion validates that TDR is an effective alternative to fusion for the treatment of discogenic low back pain in DDD patients.

Disclosures: Author 1: consultant: Aesculap; Author 2: consultant: Medtronic, K2M, stock/shareholder: Bonovo, International Spine and Orthopedic Institute, LLC, Nocimed, OuroBoros, Viscogliosi Bros Venture Partners LLC, royalties: Medtronic; Author 3: consultant: cornerstone research paid consultant fees by Aesculap; Author 4: employee; Author 5: grants/research support: Predicted, Reported, and Observed Outcomes Foundation, stock/shareholder: Nocimed, other financial report: Medtronic (vendor); Zimmer/Biomet (vendor); Nocimed (vendor); Aesculap (vendor); Author 6: consultant: Cornerstone Research Group, Inc.

\section{QF29}

\section{MODIC TYPE I CHANGES ARE NOT DUE TO HERNIATED DISC INFECTION. A PROSPECTIVE STUDY WITH FAR LATERAL ENDOSCOPIC DISCECTOMY}

Pandurang Dnyanraj Kulkarni, Christian Brogna, Prajwal Ghimire, Amanda Fife, Irfan Malik

Department of Neurosurgery, King's College Hospital, London, UK

Background: Use of antibiotics in lower back pain is supported by the theory that occurrence of Modic changes Type I in the vertebrae adjacent to an herniated disc maybe due to oedema surrounding an infected disc. This theory is based on studies conducted with open microdiscectomies. We aimed to demonstrate if the results published by Albert et al. in 2013 (1) could be replicated with an endoscopic lumbar discectomy technique.

Design: Prospective analysis.

Subjects: 60 patients with lumbar disc herniation and Modic Changes Type I in the adjacent endplates at a single level were recruited. None of the patients had previous spine surgeries nor received antibiotic treatment in the previous month.

Methods: All patients underwent a Far Lateral Endoscopic Lumbar Discectomy under standard aseptic sterile protocols. Biopsies of the extracted disc were sent for microscopy analysis, including aerobes and anaerobes standard and extended cultures.

Results: Age ranged between 25 and 66 years. Male:female ratio was 1.4:1.0. Mean time from symptoms onset was 8.7 months. $45 \%$ received an L4/5, 50\% L5/S1 and 5\% L2/3 and L3/4 far lateral endoscopic discectomy. In all cases bacteria (both aerobes and anaerobes) could not be found on microscopy nor they grew on standard and extended cultures, except in 1 case $(1.6 \%)$ where mixed anaerobes were isolated, however could represent a contamitant due to their specific characteristics.

Conclusions: Occurrence of modic changes Type 1 is unlikely to be related to an infected herniated disc. Our study demonstrates that there is not any correlation between disc infection and Modic type 1 changes. In fact, the results published by Albert et al. in 2013 (1) could not be replicated with an endoscopic lumbar discectomy technique. It can be hypothesised that the positive correlation between Modic changes and infection found in previous studies is possibly due to specimen contamination during the procedure which is less likely to happen with an endoscopic technique. Therefore, use of antibiotics in patients with Modic Type 1 changes and Low Back Pain (2) might not be clinically justified. 


\section{References}

1. Albert HB et al. (2013) Does nuclear tissue infected with bacteria following disc herniations lead to Modic changes in the adjacent vertebrae? Eur Spine J 22(4):690-696

2. Albert HB et al. (2013) Antibiotic treatment in patients with chronic low back pain and vertebral bone edema (Modic type 1 changes): a double-blind randomized clinical controlled trial of efficacy. Eur Spine J 22(4):697-707

Disclosures: Author 1: none; Author 2: none; Author 3: none; Author 4: none; Author 5: none.

\section{QF30}

\section{FACTORS ASSOCIATED WITH SYMPTOMS OF ANXIETY AND DEPRESSION IN ADULTS UNDERGOING SPINE SURGERY: A SYSTEMATIC INTEGRATIVE REVIEW}

Janni Strøm, Lene Bastrup Jørgensen, Merete Bjerrum, Cecilie Nørby Thisted, Tove Lise Nielsen, Claus Vinther Nielsen, Malene Laursen

Spine Unit, Elective Surgery Centre, Regional Hospital Central Jutland, Silkeborg, Denmark

Aim: This integrative review aims to identify factors associated with symptoms of anxiety and depression in adults undergoing spine surgery.

Background: Symptoms of anxiety and depression and their co-occurrence can be important outcome predictors of both worse pain, physical impairments, and lower health-related quality of life in patients undergoing spine surgery. In perspective; symptoms of anxiety and depression have been reported in approximately $33 \%$ of chronic back pain patients undertaking spine surgery and further emphasized in a recent study in which patients undergoing spine surgery, had an overall higher risk of postoperative depression compared to other categories of patients and conditions.

Methods: This review spans literature published 1986-2016. The search was conducted in: PubMed, CINAHL, PsycINFO, Embase, Scopus, Cochrane and Web of Science. A three-step selection and assessment process was conducted; Titles and abstracts $(\mathrm{N}=1101)$ were skimmed for relevance; the remaining articles $(\mathrm{N}=53)$ were read in full text. Studies which did not meet the inclusions criteria were excluded $(\mathrm{N}=26)$. The remaining $(\mathrm{N}=31)$ articles was critically appraised for methodological validity using JBI-MAStARI and JBI- QARI, those articles not meeting a minimum of $75 \%$ of the criteria were excluded $(\mathrm{N}=17)$. Search results consisting of 14 articles were synthesized and analyzed using a convergent qualitative design.

Results: The fourteen studies reported findings based on 4833 participants: 3017 males and 1816 females, mean age of approximately 49 years, representing predominantly Caucasians. Five categories of interacting factors associated with symptoms of anxiety and depression before and after spinal surgery were generated: (1) information, (2) pain, (3) disability, (4) return to work and (5) psychological disturbances.

Discussion: Need of information, pain, disability, return to work and psychological disturbances were found to be evident factors associated with symptoms of anxiety and depression, thereby illuminating the complexity, and interacting impact of five crucial factors. The reciprocity between anxiety and depression and the five factors challenge the use of the conventional patient self-reported outcome measurements, as the only proxy for quality of treatment.

In addition, 'lack of comprehensive information' was one of five factors with influence on the degree to which the four remaining factors influence anxiety and depression among patients going through spinal surgery, thereby stating the importance of recognizing/ developing information strategies, taking Health Literacy into account.

Conclusion: Five categories of interacting factors associated with anxiety and depression both before and after spine surgery were generated: Lack of Information, Pain, Disability, Return to work and Psychological disturbances. Information appears to have a regulating effect on anxiety and depression.

Disclosures: Author 1: grants/research support: The Independent Charity of HelseFonden; Author 2: none; Author 3: none; Author 4: none; Author 5: none; Author 6: none; Author 7: none.

\section{QF31 \\ DOES THE OUTCOME OF SURGERY FOR LUMBAR DEGENERATIVE SPONDYLOLISTHESIS DEPEND ON THE APPROPRIATENESS OF SURGERY?}

Anne F. Mannion, Valerie Pittet, John-Paul Vader, Felix Steiger, Martin Aepli, Dave O'Riordan, Francine Mariaux, Francois Porchet

Spine Center, Schulthess Klinik, Zürich, Switzerland

Introduction: In spine surgery, many treatment failures are attributable to poor patient selection and the application of inappropriate treatment. "Appropriate use criteria" (AUC) serve to help clarify the indications for a procedure. This study aimed to use recently published AUC (1) to evaluate the appropriateness of surgery for lumbar degenerative spondylolisthesis (LDS) in a large group of patients and to examine the association between adherence to the criteria and postoperative outcome.

Methods: This was a retrospective analysis of prospectively collected data recorded in our Spine Outcomes database (linked to EUROSPINE's Spine Tango Registry) from patients who had undergone LDS surgery in our clinic, 2005-2012. Appropriateness of the use of surgery in each patient was judged using the published criteria (1). Patients completed the Core Outcome Measures Index (COMI) before surgery and at 3,12 and 24 months follow-up (FU).

Results: In total, 537 patients (age $69 \pm 10$ years; 384 (72\%) women) were eligible for inclusion. $98 \%$ completed a COMI form preoperatively, $96 \%$ at 3 months FU, $92 \%$ at 12 months FU, $90 \%$ at 24 months FU. We were able to apply the appropriateness criteria in $475 / 537$ (88\%) patients with the necessary baseline data. Some type of surgery (either decompression or fusion \pm stabilisation) was considered appropriate in $224(47 \%)$ of the operated patients, inappropriate in 95 (20\%), and uncertain in 156 (33\%). There was a significant difference between the groups in the pattern of change in COMI score from preoperatively to follow-up $(\mathrm{p}<0.001)$ whereby patients who were considered appropriate (A) or uncertain (U) candidates had greater improvements in COMI than those who were considered inappropriate (I) candidates. The minimal clinically important change (MCIC) score of 2.2 points for COMI was reached by $78 \% \mathrm{~A}, 83 \% \mathrm{U}$ and $55 \%$ I cases (p $<0.001$, I vs A and U). The odds of achieving the MCIC was 3-times greater in patients considered appropriate/uncertain for surgery than in those considered inappropriate (OR 3.4, 95\% CI 1.99-5.63).

Conclusions: The results suggest a relationship between adherence to the AUC and outcome. The findings provide support for the use of AUCs in clinical practice, to guide indications for surgery, but the findings should be confirmed in prospective studies that also include a control group of non-operated patients.

(1) Mannion AF et al. (2014) Eur Spine J. 2014 Sep;23(9):1903-17. 


\section{References}

Disclosures: Author 1: none; Author 2: none; Author 3: none; Author 4: none; Author 5: none; Author 6: none; Author 7: none; Author 8: none.

\section{QF32 \\ CRITERIA FOR "SUCCESS" AFTER SURGERY FOR SPINAL STENOSIS AND DEGENERATIVE SPONDYLOLISTHESIS. A MULTICENTRE OBSERVATIONAL STUDY FROM THE NORWEGIAN REGISTRY FOR SPINE SURGERY}

Ivar M Austevoll, Margreth Grotle, Rolf Gjestad, Tore Solberg, Kjersti Storheim, Jens Ivar Brox, Erland Hermansen, Frode Rekeland, Kari Indrekvam, Christian Hellum

Department of Ortopedic, Kysthospitalet i Hagevik, Bergen, Norway

Efficacy and effectiveness of treatments in spinal surgery are most commonly assessed by Patient reported outcome measures (PROMs). A crucial consideration is the clinical importance of the results, not only statistical significance. Reporting "success"-rates will gain clinical relevant information in comparative trials as well as in registry cohorts.

Purpose: To define "success"-criteria in patients operated for lumbar spinal stenosis without spondylolisthesis (SS) and with a concomitant degenerative spondylolisthesis (DS), assessed by commonly used patient reported outcome measures (PROMs).

Methods: 4476 patients registered in the Norwegian Registry for Spine Surgery (2007-2013) were studied [3859(SS), 617(DS)]. The baseline scores and the 12 month follow-up scores for Oswestry Disability Index (ODI), Euroquol 5D (EQ-5D) and Numeric Rating Scale (NRS) for leg and back pain were analysed according to the categories in the external anchor, the Global Perceived Effect (GPE) scale. Discriminative ability were assessed by the area under the Receiver Operating Characteristic (ROC) curves for those reporting "success" ("completely recovered' and 'much improved') and "nonsuccess" ('slightly improved', unchanged', 'slightly worse', 'much worse', and 'worse than ever'). We evaluated the follow-up score (raw score at 12 month), the numerical change score (baseline score minus 12 month follow-up score), and the percent change score (change score in percent of baseline score) to identify the cut-off values that maximised the percentage of patients correctly classified as "success" according to the anchor. Analyses were performed separately for SS and DS.

Results: We found good or excellent test accuracy for the three candidate scores for all PROMs [(Area under the curve (AUC) from 0.82 to $0.92(1.0=$ perfect accuracy $)]$ but not for the EQ-5D's numerical change score [AUC 0.76 (fair accuracy)]. The percentage of correctly classified patients were in general lower and more dependent on the baseline scores for the numerical change score than for the follow-up score and the percent change score.

The estimated cut-off values were identical for patients with and without spondylolisthesis, except for the numerical change score for NRS back pain:

ODI: follow-up score $\leq 24$, numerical change score $\geq 10$, percent change score $\geq 30 \%$.

EQ-5D: follow-up score $\geq 0.69$, numerical change score $\geq 0.11$.

NRS leg pain: follow-up score $\leq 3$, numerical change score $\geq 3$, percent change score $\geq 40 \%$.

NRS back pain: follow-up score $\leq 4$, numerical change score $\geq 2$ for SS and $\geq 3$ for DS, percent change score $\geq 40 \%$.

Conclusion: We recommend to use the follow up score or percent change scores for calculating "success" rates after surgery for spinal stenosis with and without degenerative spondylolisthesis. Cut-offs on the numerical change score were less accurate and more dependent on the baseline score.

Disclosures: Author 1: none; Author 2: none; Author 3: none; Author 4: none; Author 5: none; Author 6: none; Author 7: none; Author 8: none; Author 9: none; Author 10: none.

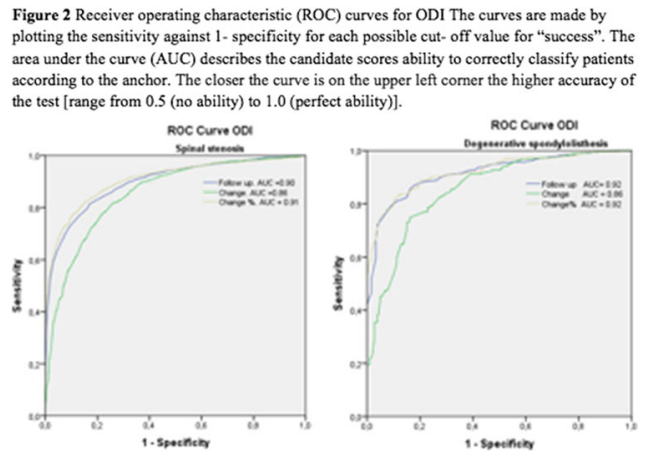

\section{QF33 \\ CREATING LORDOSIS IN TRANSFORAMINAL AND POSTERIOR LUMBAR INTERBODY FUSION (TLIF/PLIF): THE IMPACT OF SURGICAL TECHNIQUE: A CONTROLLED CADAVERIC STUDY}

Peter Robertson, Will Armstrong, Daniel Woods, Jeremy Rawlinson

The Orthopaedic Clinic, Mercy Specialist Centre, Auckland, New Zealand

Introduction: Transforaminal and Posterior Lumbar Interbody Fusion (TLIF/PLIF) are widely used for lumbar spinal fusion, anterior column support and potentially lordosis control. Recreating lordosis is increasingly recognized as a critical factor in these procedures. The advantage of TLIF vs PLIF and the effect of various surgical stages between a unilateral and bilateral procedure are unknown. This study performed a controlled stepwise surgical simulation of cadaveric TLIF/PLIF to assess the impact of progressive surgical intervention. Method: Eight cadaveric motion segments (four at L3/4 and four at L5/S1) underwent stepwise-simulated surgery. The steps progressed from a unilateral TLIF ( $18^{\circ}$ lordosis, $27 \mathrm{~mm}$ length) at three stages of resection (unilateral facetectomy, bilateral facetectomy, then a wide posterior decompression/posterior column osteotomy) to a bilateral PLIF (22 mm length) with $18^{\circ}$ then $24^{\circ}$ lordotic cages. Dorsal compression across pedicle screws (bicortical and cement reinforced to resist loosening on multiple retesting) and rods provided stability before segmental lordosis was captured with lateral radiography. Repeated-measures ANOVA was used to compare across specimens and procedures.

Results: Pre-operative lordosis (pooled) was $8.6^{\circ} \pm 6.1^{\circ}$ $\left(\right.$ mean $\pm \mathrm{SD}$ ), and maximum post-surgical lordosis was $20.4^{\circ} \pm 6.3^{\circ}$ with the $24^{\circ}$ cage. No statistical increase in lordosis was achieved with unilateral TLIF and minimal bone resection $\left(10.1^{\circ} \pm 6.3^{\circ}, 18 \%\right.$ increase, $p=0.9$ ). Stepwise increase in lordosis occurred with progressive bone resection from unilateral TLIF with bilateral facetectomy (50\%) and posterior wide decompression/posterior column osteotomy $(12 \%)$ to bilateral PLIF $(18 \%)$ with the $18^{\circ}$ cage $\left(15.2^{\circ} \pm 8.2^{\circ}, 17.0^{\circ} \pm 4.7^{\circ}\right.$, to $20.1^{\circ} \pm 6.3^{\circ}$, respectively). The techniques with wide posterior decompression had significantly greater lordosis than the pre-operative and unilateral TLIF conditions $(\mathrm{p}<0.01)$. 
Discussion: This cadaveric study demonstrated that operation type (TLIF v PLIF), posterior bone resection, and implant selection influence lordosis control in the lumbar spine. PLIF created more lordosis than TLIF, although TLIF lordosis can be increased with progressive bone resection of contralateral facet and midline structures. This cadaveric study strongly suggested, that along with lordotic cages, operation type and extent of bone resection contribute to lordosis control in interbody fusion using TLIF/PLIF techniques. Disclosures: Author 1: consultant: Medtronic, NuVasive; Author 2: stock/shareholder: Medtronic, employee: Medtronic; Author 3: stock/ shareholder: Medtronic PLC, employee: Medtronic PLC; Author 4: employee: Medtronic Spine.

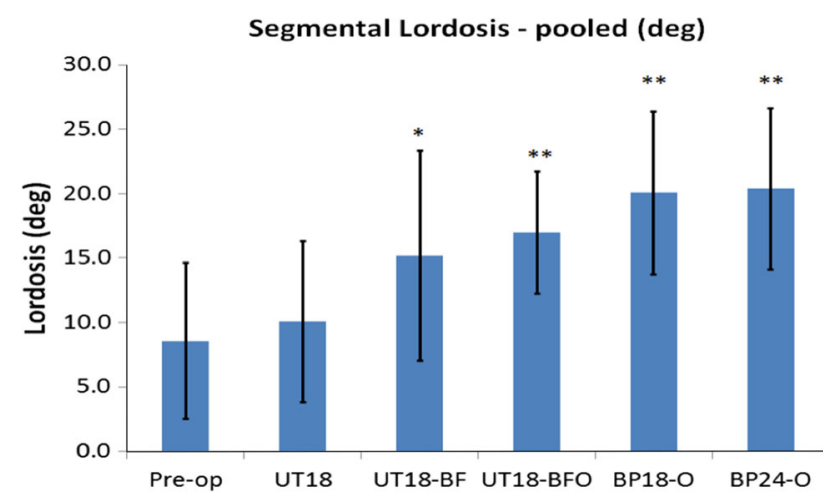

\section{QF34 \\ ADJACENT SEGMENT DEGENERATION AND TOPPING OFF: NEVER STOP AT THE APEX!}

Markus Konieczny, Shejda Mokhaberi, Ruediger Krauspe

Department of Orthopedic Surgery, University Hospital of Duesseldorf, Duesseldorf, Germany

Background: There is still no consensus in the literature if Topping off by a rod based dynamic posterior stabilization device reduces the rate of adjacent segment degeneration (ASD).

Apical segments of the Lumbar Lordosis (LL) are reported to be significantly more mobile than other segments. In the light of these data, choosing lumbar apical vertebra (LAV) as upper instrumented vertebra (UIV) may lead to a higher rate of ASD due to increased mobility as consequence of the combined effects (apex of LL and adjacent segment to rigid fixation) which subsequently increases the strain on the intervertebral discs and most probably also on the facet joints at this level.

Purpose: To investigate if applying the Transition system $\left({ }^{\circledR}\right.$ Globus Medical) as topping off can prevent ASD and if rate of ASD is increased if UIV is LAV.

Materials and methods: We conducted a Retrospective Study on 134 consecutive patients who have been operated by instrumented fusion of the lumbar spine from 01.2010 to 02.2013 in a single spine center. One end-point of the study was set when patients needed Revision Surgery. Mean follow up was 20 months (StE 1.1). 35 patients were lost to follow up. Thirty patients were treated with topping of by hybrid instrumentation (Group 1), 69 patients had the standard procedure without topping off technique (Group 2).

Patients were routinely examined at 6 weeks, 12 weeks, 12 months and 24 months post-operative by clinical and radiological examination.

Statistical analyze was performed by SPSS 23:
Descriptive data are presented as mean and standard error (StE). We conducted a one sided Chi Square test to analyze if hybrid instrumentation, level of UIV in relation to Apex of LL (LAV) and preoperative degree of degeneration of the adjacent segment were related to rate of ASD. ASD was diagnosed if Weiner grade increased by one or more levels during the follow-up period.

Significance level was set at $\mathrm{p}<0.05$, bonferroni adjustment was applied.

Results: 84 of 99 patients (84\%) were classified as being multimorbid.

$56 / 99$ patients (56\%) developed ASD, 18 patients of group $1(60 \%)$ and 38 patients of group $2(55 \%)$. The difference was not significant $(\mathrm{p}<0.05)$.

Seven patients underwent revision surgery for ASDis, one patient in group 1 and six patients in group 2 .

In 17 patients (17\%) UIV was at the level of LAV (instrumentation stopped at apex of LL). 14/17 patients (82\%) developed and ASD. The difference was significant $(\mathrm{p}<0.05)$.

Conclusions: Instrumented fusion of the lumbar spine should not stop at the apex of the lumbar curve. Topping off by hybrid dynamic fixation does not reduce the rate of ASD and may only be considered in special indications.

Disclosures: Author 1: none; Author 2: none; Author 3: other financial report: Payed Lecturer Globusmedical.

\section{QF35 \\ LONG-TERM EFFICACY OF INTERLAMINAR STABILIZATION IN MEDICARE PATIENTS}

Christina Dowe, Samuel Grinberg, Rachel Simon, Frank Cammisa, Celeste Abjornson

Hospital for Special Surgery, New York, NY, USA

Background: The number of complex fusions performed on elderly ( $\geq 65$ years) patients with spinal stenosis with or without spondylolisthesis is increasing. Interlaminar stabilization (ILS) offers a nonterminal surgery option between decompression alone and decompression with fusion. ILS has been shown to have durable outcomes when compared to posterolateral fusion for stenotic patients with up to a Grade I spondylolisthesis. The purpose of this study was to examine the efficacy ILS in the elderly population through comparison to ILS in younger patients and fusion in older patients.

Methods: Under FDA-regulated Investigational Device Exemption (IDE) study, a total of 322 patients from 21 sites in the US were enrolled between 2006 and 2010 in the prospective, randomized trial. All patients were diagnosed with spinal stenosis with or without spondylolisthesis at either 1-level or 2-levels. Of the 322 patients enrolled in this study, 131 patients under the age of 65 years underwent decompression with ILS, 84 patients over the age of 65 years $(<65)$ underwent decompression with ILS and 57 patients $\geq 65$ underwent decompression with fusion. Composite clinical success (CSS) was used to measure of the overall efficacy of the different treatments. CSS was determined using Oswestry Disability Index (ODI), the absence of reoperations or epidurals, the absence of persistent or new neurological deficits and the absence of major devicerelated complications. The patients were assessed before and after surgery at 6 weeks and 3, 6, 12, 18, 24, 48 and 60 months. Clinical assessment included patient self-assessment, physical and neurological examination, and radiographic evaluation. The results of the different assessments were combined to calculate the percentage of patients achieving composite clinical success.

Results: At 60 months, there were no statistically significant differences in CCS or any of the components of CCS between the $<65$ and 
$\geq 65$ ILS groups. There was no difference in mean operative time or blood loss, but $\geq 65$ patients spend an average of 2.07 days in the hospital compared to 1.79 days for the younger patients $(\mathrm{p}=0.013)$. Similarly, at 60 months, there were no statistically significant differences in CCS or any of the components of CCS between the $\geq 65$ ILS and fusion groups but there were statistically significant differences in all intraoperative componets: ILS patients experienced less blood loss $(p<0.001)$, shorter surgeries $(p<0.001)$ and a shorter hospital stay $(\mathrm{p}<0.001)$ than fusion patients.

Conclusions: ILS elderly patients experienced less blood loss, a shorter operation and shorter hospital stay than fusion elderly patients. Clinically, there were no significant differences in clinical success of elderly patients when compared with younger patients receiving ILS. Disclosures: Author 1: none; Author 2: none; Author 3: none; Author 4: grants/research support: Bacterin, Integra, NuTech, Vertical, Spinal Kinetics, Centinel, Mallinckrodt, consultant: 4Web Medical, Alphatec Spine, Inc., Healthpoint Capital Partners, LP., IVY Healthcare Partners, LP., Nuvasive, Inc., Paradigm Spine, LLC., Spinal Kinetics, Spinal Partners III, Vertebral Technologies, Inc., Vertical Spine, Viscogliosi Brothers, LLC, stock/shareholder: Alphatec Spine, Inc., BI Members, LLC, BioAssets Development Corp., Healthpoint Capital Partners, LP., Ivy Healthcare Partners, LP., Nuvasive, Inc., Orthopaedic Investment Partners, LP., Paradigm Spine, LLC., Promethean Surgical Devices, Scient'x USA, Spinal Kinetics, Vertebral Technologies, Inc., Viscogliosi Brothers, LLC., Vertical Spine, royalties: Nuvasive, Inc, other financial report: Bonovo Orthopedics, Inc., Liventa BioSciences, Inc., MMF Systems, Inc., Royer Biomedical, Inc., Woven Orthopedic Technologies; Author 5: consultant: Paradigm Spine.

\section{QF36 \\ ADJACENT LEVEL STENOSIS OR RECURRENT STENOSIS MORE COMMON AFTER DECOMPRESSION WITH FUSION FOR LUMBAR SPINAL STENOSIS THAN AFTER DECOMPRESSION ALONE}

\section{Thomas Karlsson, Peter Försth, Bengt Sandén}

Department of Orthopedics, Uppsala University Hospital, Uppsala, Sweden

Magnetic Resonance Image results 2 years postop from the Swedish Spinal Stenosis Study, a multi-center RCT of 211 patients.

Summary: We randomized 211 patients with lumbar spinal stenosis at one or two adjacent levels to surgery with decompression only or decompression with fusion. MRI 2 years post-op shows overall higher risk for developing restenosis or stenosis at proximal adjacent level after decompression with fusion than after decompression alone.

Background: Decompression alone is considered to cause recurrent stenosis. Decompression with fusion is considered to cause adjacent level stenosis. We investigated if this is true and which method causes the overall highest rate of stenosis 2 years postoperatively.

Materials and methods: From 2006 to 2012, 211 patients aged 50-80 from six hospitals were enrolled. Inclusion criteria were dural sac area $\leq 75 \mathrm{~mm}^{2}$, pseudoclaudication in one/both legs VAS $>30$, back pain VAS $>30$ and duration of symptoms $>6$ months.

Patients were randomized to decompression only or decompression with fusion, and stratified for pre-op degenerative spondylolisthesis of $\geq 3 \mathrm{~mm}$ on plain X-ray. Method for decompression and fusion was determined by the surgeon.

178 patients were followed up after 2 years with MRI of the operated level(s) and proximal adjacent level. 177 were evaluated for distal adjacent level stenosis. Primary outcome measure was dural sac cross area $\leq 75 \mathrm{~mm}^{2}$. Secondary outcome measures were absolute dural sac area and stenosis according to Schizas, in which Schizas grade C and D was considered stenotic. Statistic methods used were Chi square, logistic regression and t-test.

Results: The overall risk for developing restenosis or stenosis at proximal adjacent level after 2 years was higher after decompression with fusion than after decompression alone. This was due to a higher rate of adjacent level stenosis after additional fusion. The rate of recurrent (same level) stenosis was somewhat lower for patients treated with additional fusion, although not significant. The presence of pre-operative olisthesis did not influence the results.

The distal adjacent level was most often L5-S1, which is often $\leq 75$ $\mathrm{mm}^{2}$ on healthy individuals. None of the patients had Schizas C-D, and the distant adjacent level was therefore not included in the analysis.

Conclusion: In this multi-center randomized controlled MRI study we found that adding fusion to decompression surgery in lumbar spinal stenosis increases the overall risk of developing recurrent (same level) stenosis or proximal adjacent level stenosis 2 years postop. This supports clinical studies recommending decompression without fusion as first method of choice for lumbar spinal stenosis. Disclosures: Author 1: none; Author 2: none; Author 3: none.

\begin{tabular}{|c|c|c|c|c|c|c|c|}
\hline & & & \multicolumn{2}{|c|}{ Decompression } & \multicolumn{2}{|c|}{ Dec with fusion } & \\
\hline All patients (n) & & & 87 & & 9 & & \\
\hline \multicolumn{2}{|c|}{ Proximal adjacent } & aren $<75 \mathrm{~mm}^{2}(\mathrm{n})$ & & & & & \\
\hline \multirow{2}{*}{ level stenosis } & & \begin{tabular}{|l}
$\mid$ area $\leq 75 \mathrm{~mm}^{2}(\mathrm{n})$ \\
Schizas C-D (n)
\end{tabular} & $\begin{array}{l}16 \\
14\end{array}$ & $\frac{18 \%}{16 \%}$ & $\frac{4}{3}$ & $\begin{array}{l}45 \% \\
40 \% \\
\end{array}$ & $\begin{array}{l}\mathrm{p}=0,0002 \\
\mathrm{p}=0,0005\end{array}$ \\
\hline & & mean area $\left(\mathrm{mm}^{2}, \mathrm{sd}\right)$ & 123 & 48 & 9 & 44 & $p<0,0001$ \\
\hline & & & & & & & \\
\hline \multicolumn{2}{|c|}{ Recurrent (same level) } & area $\leq 75 \mathrm{~mm}^{2}(\mathrm{n})$ & 12 & $14 \%$ & & $5.5 \%$ & $p=0,06$ \\
\hline \multirow[t]{2}{*}{ stenosis } & & Schizas C-D (n) & 7 & $8 \%$ & & $1 \%$ & $p=0,056$ \\
\hline & & mean area $\left(\mathrm{mm}^{2}, \mathrm{sd}\right)$ & 128 & 46 & 19 & 59 & $p<0,0001$ \\
\hline \multicolumn{2}{|c|}{ Overall post-op stenosis } & area $\leq 75 \mathrm{~mm}^{2}(\mathrm{n})$ & 25 & $29 \%$ & 4 & $46 \%$ & $p=0,016$ \\
\hline \multirow{2}{*}{\multicolumn{2}{|c|}{$\begin{array}{l}\text { (proximal adjacent and/or } \\
\text { recurrent) }\end{array}$}} & Schizas C-D (n) & 20 & $23 \%$ & 3 & $40 \%$ & $p=0,017$ \\
\hline & & mean area $\left(\mathrm{mm}^{2}, \mathrm{sd}\right)$ & 99 & 35 & 9 & 43 & $p=0,3$ \\
\hline
\end{tabular}

\section{QF37}

\section{DISC HEIGHT RESTORATION WITH EXTREME LATERAL INTERBODY FUSION: DO WE NEED TO DIRECTLY} DECOMPRESS THE NERVE ROOTS?

\section{Salvatore Russo, Khai Lam}

Department of Neurosurgery, The National Hospital for Neurology and Neurosurgery, London, UK

Introduction: Loss of disc height in the lumbar spine may lead to foraminal and central canal stenosis causing radiculopathy. Neuronal decompression is one of the goals of surgery. This is traditionally achieved by direct exploration and decompression of the neuronal elements. Extreme lateral interbody fusion (ELIF) is a minimally invasive technique for anterior column stabilisation of the thoracolumbar spine. ELIF is particularly effective in disc height restoration and segmental realignment and can be used to indirectly decompress the neuronal elements without adding the morbidity associated with direct root exploration.

Purpose of the study: Evidence on efficiency and safety of ELIF in treating radiculopathy are lacking. The purpose of this study is to evaluate whether ELIF can indirectly decompress the neuronal elements and improve functional outcomes in degenerative lumbar stenosis.

Materials and methods: 103 consecutive patients operated with ELIF between 2012 and 2016 were included in a retrospective study. 
All patients had degenerative lumbar disorders with either central canal or foraminal stenosis.

Pre-operative, immediate postoperative and last follow-up radiological and clinical outcomes were collected. X-ray, CT and MRI of the lumbar spine were used to measure average disc height, segmental angulation, lumbar lordosis, foraminal height, central canal and foraminal area. Clinical outcomes were evaluated by means of visual analogue scale (VAS) for back pain and leg pain, Oswestry Disability Index (ODI) and EQ-5D

Results: 149 lumbar levels were operated with ELIF. 81.6\% (84/103 patients) had posterior stabilisation with bilateral pedicle screws, $5.8 \%(6 / 103)$ had unilateral pedicle screws on the symptomatic site only and $12.6 \%(13 / 103)$ were treated with stand-alone cages. The primary diagnosis was disc degenerative disease in $56 \%$ of patients, spondylolisthesis in $25 \%$ and degenerative scoliosis in $19 \%$. The average follow-up was 10.6 months.

Postoperative disc height, segmental angulation and lumbar lordosis improved, respectively by 63,28 and $14 \%(\mathrm{p}<0.0001)$. Foraminal height and area on the stenotic site increased by 28 and $63 \%$, respectively and by 23 and $41 \%$ on the contralateral foramen $(\mathrm{p}<0.0001)$. The central canal area increased postoperatively by $44 \%(\mathrm{p}<0.0001)$.

All functional outcomes improved post-operatively $(\mathrm{p}<0.0001)$. VAS for back pain and leg pain improved by 60.6 and $74.6 \%$, respectively. ODI and EQ-5D increased by 49.7 and $25.8 \%$, respectively.

Conclusion: ELIF can restore foraminal height and central canal area in degenerative lumbar disorders avoiding direct roots exploration and disruption of the posterior elements of the spine. Moreover, ELIF is clinically effective and it is associated with significant improvement in functional outcome scores. To our knowledge, this study is one of the largest ELIF retrospective case-series that aim to investigate clinical and radiological outcomes in degenerative lumbar stenosis.

Disclosures: Author 1: none; Author 2: none.

\section{QF38 \\ RADIOLOGICAL CHANGES AND PERIOPERATIVE COMPLICATIONS AFTER OBLIQUE LATERAL INTERBODY FUSION AT L4-5; PRELIMINARY REPORTS FOR COMPARISON WITH THE CLASSICAL MINIMALLY- INVASIVE ALIF AT L4-5}

Nam-Su Chung, Chang-Hoon Jeon, Han-Dong Lee, Young-Uk Seo

Department of Orthopaedic, Ajou Univercity Medical Center, Suwon, South Korea

Purpose: We aimed to compare the radiological outcome and safety between oblique lateral interbody fusion (OLIF) and classical minimally-invasive anterior lumbar interbody fusion (ALIF) at the L4-5 segment.

Introduction: OLIF is equivalent to perform a lateral lumbar interbody fusion with antero-lateral retroperitoneal approach. Cage for OLIF has advantages over cage for ALIF in that the larger lateral cage can achieve greater coverage of fusion area, greater restoration of disc height and more indirect decompression. However, few studies have compared the post-operative change of dimension and the perioperative complications between OLIF and ALIF

Methods: This was a retrospective study of 55 consecutive patients who underwent OLIF at the L4-5 segment and a control cohort of 48 consecutive patients who underwent ALIF at the same segment. Parameters of radiological measurement were the anterior and posterior disc heights, the coronal and sagittal disc angles, the height of foramen, and the cross-sectional area (CSA) of foramen using computed tomography at preoperative and postoperative 3 months. The occurrence of perioperative complications was also compared. Results: Baseline demographics and pre-operative radiological parameters were similar between the two groups (all $\mathrm{P}>0.05$ ). The mean increase in anterior disc height of the OLIF group was $4.5 \pm 3.2 \mathrm{~mm}$, which was significantly higher than that of the ALIF group $(2.4 \pm 2.1 \mathrm{~mm})(\mathrm{P}=0.025)$. The mean increase in posterior disc height of the OLIF group was $4.5 \pm 2.4 \mathrm{~mm}$, which was significantly higher than that of the ALIF group $(1.5 \pm 4.3 \mathrm{~mm})$ $(\mathrm{P}=0.010)$. There was no difference in the postoperative change with respect to the coronal and sagittal disc angles and the height of foramen, and the cross-sectional area (CSA) of foramen between the OLIF and ALIF groups (all $\mathrm{P}>0.05$ ). There were no major perioperative complications in either group. Thigh pain ( 3 cases) and thigh numbness (4 cases) were observed in the OLIF group only, but those symptoms disappeared at the postoperative 3-month follow-up.

Conclusions: OLIF performed at the L4-5 segment has advantages over ALIF in terms of a greater increase in anterior/posterior disc height, while the segmental angular change, increase and the height of foramen, and the cross-sectional area (CSA) of foramen and perioperative complications were comparable between the two techniques. Disclosures: Author 1: none; Author 2: none; Author 3: none; Author 4: none.

\section{QF39 \\ REVISION SURGERY OF SYMPTOMATIC PSEUDARTHROSIS AFTER LUMBAR FUSION: A VIABLE TREATMENT OPTION?}

Markus Loibl, Anne Mannion, Siegmund Lang, Frank Kleinstück, Tamas Fekete, Dezsö Jeszenszky, Daniel Haschtmann

Spine Center, Schulthess Klinik, Zürich, Switzerland

Introduction: Pseudarthrosis is a feared complication after lumbar fusion that can be associated with pain and disability. The aim of the present study was to investigate outcome, surgical strategy, and preoperative radiographic parameters in patients undergoing revision surgery for symptomatic pseudarthrosis after short lumbar fusion.

Methods: This was a retrospective analysis of prospectively collected data from 91 patients (49 male, 42 female; $54.8 \pm 12.6$ years at surgery) who had undergone revision surgery between 2005 and 2014 . Inclusion criteria were prior lumbar fusion ( $\leq 3$ segments), symptomatic pseudarthrosis, and at least 6 months' follow-up. Radiographic and surgical data were retrieved from the patient charts. The multidimensional Core Outcome Measures Index (COMI) was completed by the patients, pre and postoperatively, with score differences being analyzed using repeated measures ANOVA.

Results: The mean duration until revision was $35.9 \pm 41.4$ months. $50 / 91$ patients $(55 \%)$ had originally been treated at our spine center, and 41/91 (45\%) at other hospitals. In 90/91 (99\%) patients, posterior fusion had been performed with pedicle or translaminar screws. In $58 / 91(64 \%)$ patients, the initial interbody fusion was performed with a cage. Screw loosening/breakage was demonstrable in 50/91 (55\%) pre-revision radiographs, and 45/62 (73\%) available CT images; cage loosening was detected in 21/58 (36\%) and 17/58 (29\%), respectively. TLIF was used as part of the revision strategy in 76/91 (84\%) patients, and PLIF in $8 / 91$ (9\%). Screws were exchanged in 90/90 (100\%) and cages in $24 / 58(41 \%)$. BMP was used in 18/91 (20\%), and iliac bone graft in 55/91 (60\%) patients. 15/91 (16\%) patients underwent further revision during the 2-y follow-up. 
The COMI revealed a small but statistically significant improvement $(\mathrm{p}<0.01)$ from $8.6 \pm 1.2$ pre-revision to $6.6 \pm 2.4,6.6 \pm 2.7$ and $6.6 \pm 2.6$ at 3, 12 and 24 months after, respectively. At 24 months, $30 / 79(38 \%)$ had achieved the COMI minimal clinically important change (MCIC) score of 2.2. points.

Discussion: Revision surgery for symptomatic pseudarthrosis resulted in a small statistically significant improvement in the group mean COMI score, but the proportion reaching a clinically important change after 24 months was low. Revision surgery represents a viable treatment option for some patients. However, both patients and surgeons should be aware of the potentially marked, ongoing impairment at 2 years' follow-up.

Disclosures: Author 1: none; Author 2: none; Author 3: none; Author 4: grants/research support: Depuy-Synthes; Author 5: none; Author 6: consultant: DePuy Synthes Spine, royalties: DePuy Synthes Spine; Author 7: none.

\section{QF40 \\ PEDICLE SCREW DIAMETER AFFECTS THE UNION RATE OF SINGLE LEVEL L4/5 TRANSFORAMINAL LUMBAR INTERBODY FUSION}

Bungo Otsuki, Shunsuke Fujibayashi, Shimei Tanida, Shuichi Matsuda

Department of Orthopedics, Kyoto University, Kyoto, Japan

Object: One of major complications after transforaminal or posterior lumbar interbody fusion (TLIF or PLIF) with posterior pedicle screw (PS) instrumentation technique is pseudoarthrosis, which sometimes causes back and/or radicular pain. We hypothesized that the use of PSs with narrower PS diameter (PSD) than the inner diameter of pedicle will be a risk factor of pseudoarthrosis after TLIF.

Methods: All patients who underwent L4/5 single level TLIF from Oct 2011 to Feb 2015 using conventional open approach or paraspinal approach were retrospectively reviewed. We excluded the patients with the age $<50$ years, Parkinson's disease, collagen disease, active malignancy, hemodialysis, or diffuse idiopathic skeletal hyperostosis (Otsuki B, ESJ 2015). The shortest minor axis of inner pedicle (SMAIP) was measured using reconstructed axial image of computed tomography of each pedicle. We defined filling index (FI) as SMAIP $^{2}-$ PSD $^{2}$ and total FI (TFI) as sum of each FI (FI of bilateral L4 and L5) (Figure). Pseudoarthrosis was diagnosed at 1 year after the surgery using CT and dynamic radiographs (Fujibayashi, Spine 2012). There was no patient with surgical site infection and/or iatrogenic durotomy in this series.

Results: Total 40 patients with the mean age of 67 were analyzed (17 men and 23 women). Nine patients were diagnosed as pseudoarthorosis at 1 year.

Age and sex were not significantly different between the pseudoarthrosis group and the union group, however; TFI was significantly larger in the pseudoarthrosis group than that of the union group ( 253.35 vs $75.7, \mathrm{P}<0.0001)$. Logistic regression analysis also showed that TFI was an independent significant factor for predicting pseudoarthorosis.

Discussion: Numerous risk factors have been reported in the development of pseudoarthorosis, including length of the fusion site, fusion technique, smoking, infection and durotomy. However, there is no study about the effect of PSD on pseudoarthorosis. In this study, we selected the patients with single same level TLIF (L4/5) to exclude the effect of fusion length and fusion site, and large TFI has clarified as a risk factor for pseudoarthorosis for the first time. This indicates that selecting larger diameter PS will decrease the rate of pseudoarthorosis. We believe that surgeon should measure the SMAIP before surgery and select proper PS size. The limitations of the study are small number of the patients, and without the data of smoking and bone mineral density.

Conclusion: The use of narrow diameter PS compared to the actual pedicle diameter is a risk factor for pseudoarthorosis after TLIF. Surgeon should measure the true diameter of pedicle before surgery and select appropriate PS size.

Disclosures: Author 1: none; Author 2: none; Author 3: none; Author 6: none.
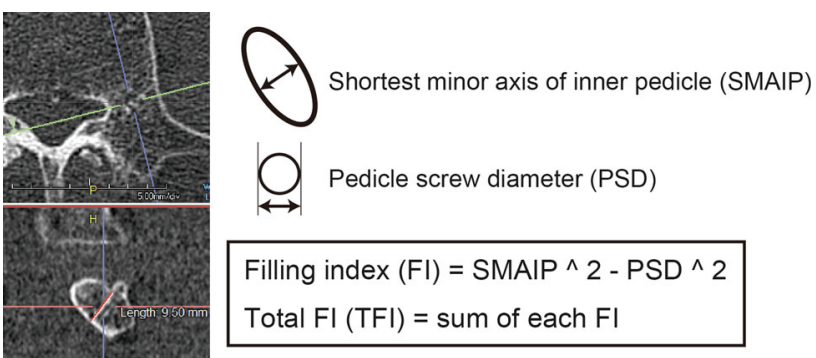

Filling index $(F I)=\operatorname{SMAIP}^{\wedge} 2-\mathrm{PSD}^{\wedge} 2$

Total FI $(\mathrm{TFI})=$ sum of each $\mathrm{FI}$

Figure. Shortest minor axis of inner pedicle (SMAIP) was measured using multi planar reconstruction. Filling index $(\mathrm{FI})$ and total $\mathrm{FI}(\mathrm{TFI})$

was measured as in the figure.

\section{QF41 \\ BULKY BONE GRAFT VERSUS GRANULAR BONE GRAFT IN LUMBAR FACET JOINT FUSION: A PROSPECTIVE SELF-CONTROLLED COMPARATIVE STUDY}

\section{Bolong Zheng, Baorong He, Dingjun Hao}

Department of Spine Surgery, Xi'an, China

Background: Lumbar facet joint fusion is one of the fusion strategies in degenerative lumbar diseases, in which the bone graft can be bulky or granular. But which grafting technique is better remains unclear. Some Authors have reported granular bone instead of bulky bone graft in facet joint fusion with satisfactory results. However, the assessment of fusion is different among studies. To our knowledge, no prospective self-controlled comparative study has compared the efficacy of bulky and granular bone graft in lumbar facet joint fusion by CT.

Purpose: To compare the efficacy of bulky and granular bone graft in lumbar facet joint fusion, and evaluate the fusion characteristics in the short and long term.

Methods: From June 2010 to December 2012, 135 consecutive patients with degenerative lumbar diseases underwent lumbar facet joint fusion in our spine center. Bulky bone graft and granular bone graft were randomly allocated to either side of bilateral facet joints, with both gotten from decompressed lumbar lamina. In the bulky bone graft group, we used a wedge-shaped and double-faced cancellous bone for facet joint fusion. In granular bone graft group, we used morcellated bone stuck in facet joint. Patients were followed up every 3 months within 1 year and every 6 months beyond 1 year. CT was recorded at each follow-up, different grades of fusion and fusion rate (defined as Grade III and Grade IV) were recorded.

Results: The operations were successful in one hundred and thirtyfive patients, with all showing improvement in clinical symptoms. The mean follow-up time was 28.7 months (ranged 10-35 months). One hundred and twenty-eight patients were followed-up for more than 2 years, including 73 men and 55 women, mean age 43.8 years 
(ranged 38-58 years), with follow-up rate of $94.8 \%$. At 3 months follow-up, bulky bone graft group showed Grade I,11/128 (8.6\%), Grade II, 31/128 (24.2\%), Grade III, 76/128 (59.4\%), Grade IV, 10/128 (7.8\%); granular bone graft group showed Grade I, 71/128 (55.5\%), Grade II, 48/128 (37.5\%), Grade III, 6/128 (4.7\%), Grade IV, $3 / 128(2.3 \%)$. The fusion rate of bulky bone graft is higher than granular bone graft group $(67.2$ vs $7.0 \%, \mathrm{P}<0.01)$. At the last follow-up, bulky bone graft group showed Grade I, 4/128 (3.1\%), Grade II, 17/128 (13.3\%), Grade III, 42/128 (32.8\%), Grade IV, 65/128 $(50.8 \%)$; granular bone graft group showed Grade I, 21/128 (16.4\%), Grade II, 44/128 (34.4\%), Grade III 31/128 (24.2\%), Grade IV, $32 / 128(25.0 \%)$. The fusion rate of bulky bone graft is higher than granular bone graft group ( 83.6 vs $49.2 \%, \mathrm{P}<0.05$ ). No pedicle screw loosening, transition or breakage occurred.

Conclusions: In lumbar facet joint fusion, compared with granular bone graft, bulky bone graft can achieve shorter fusion time, higher fusion rate in short and long term. The assessment of fusion based upon flexion-extension radiographs is not reliable. Confirmation of fusion should be based upon fusion grading system on CT image.

Disclosures: Author 1: none; Author 2: none; Author 3: Author 4: Author 5: Author 6: Author 7: Author 8: Author 9: Author 10.

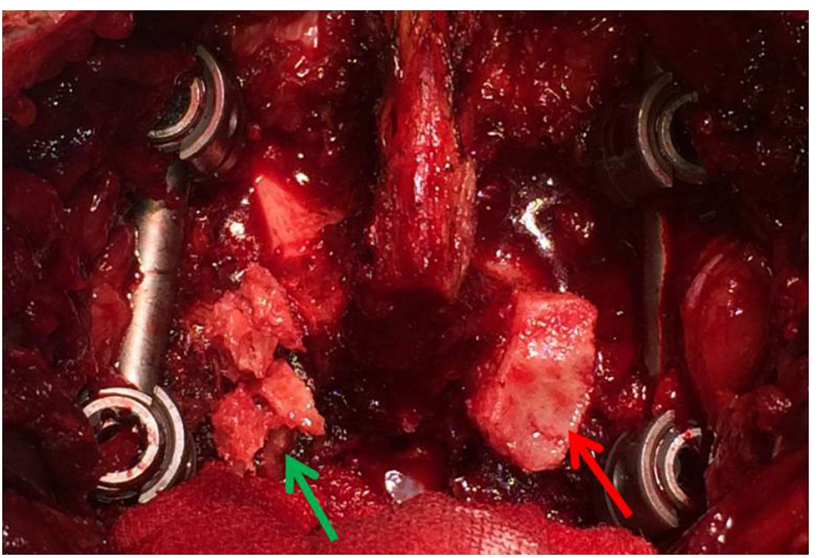

Fig. 1 The green arrow indicates granular bone graft, and the red arrow indicates bulky bone graft.

\section{NEW TECHNIQUES AND IMAGING}

\section{QF42 \\ NEW SURGICAL METHODS IN A HEALTH ECONOMIC PERSPECTIVE}

Oddrun A. Fredriksli, Greger Loenne, Hege Andresen, Oystein P. Nygaard, Lars G. Johnsen

Department of Neurosurgery St. Olavs Hospital/NTNU, Norwegian University of Science and Technology, Trondheim, Norway

Background/introduction: Because of limited resources, the health Authorities must prioritize between many treatment options. The direct and indirect costs associated with neck and back pain in Norway, were between 1.5 and 2 billion Euros in 2008. Effectiveness, in the sense of costs saved and at the same time utility for the patient in the form of health gained, is most wanted. Health economic evaluations can be used to identify, measure, value and compare the costs and the consequences of choosing competing alternatives.
Materials and methods: We have performed three multicentre randomized controlled trials (RCTs) with comprehensive collections of healthcare utilization data:

1. Cost-effectiveness of total disc replacement vs multidisciplinary rehabilitation in patients with chronic low back pain.

2. Cost-effectiveness of X-stop vs minimally invasive decompression in lumbar spinal stenosis.

3. Cost-effectiveness of arthroplasty vs fusion in treatment for cervical radiculopathy.

The outcome scores of general health-related quality of life questionnaires such as the EQ-5D-3L are combined into different health states. Since these quantitative values represent a valuation or preference of health states for the patients, they are called utility indexes. Combined with the period of follow-up, health utility indexes are used to calculate quality adjusted life years (QALYs).

Cost-effectiveness is evaluated by calculating the difference in mean costs divided by the difference in mean QALYs gained. The result is presented as ICER-incremental cost-effectiveness ratio. This means the cost per unit of effect gained using the new treatment instead of the existing (standard) treatment.

Results:

1. An RCT comparing total disc replacement (TDR) with multidisciplinary rehabilitation (MDR) in 173 patients with chronic low back pain. TDR was cost-effective compared with MDR after 2 years when using EQ-5D-3L for assessing QALYs gained and a willingness to pay of $€ 74,600$ (NOK 500,000)/QALY.

2. An RCT comparing X-stop with minimally invasive decompression in 96 patients with symptomatic lumbar spinal stenosis. With a (nonsignificant) higher effect, there was a $50 \%$ likelihood that $\mathrm{X}$-stop is cost-effective at the extra price of $€ 25,700$ (ICER) for a QALY compared with minimally invasive decompression after 2 years. The major disadvantage with $\mathrm{X}$-stop was the high risk of secondary surgery.

3. An RCT comparing arthroplasty with fusion in 136 patients with cervical radiculopathy, showed that arthroplasty was not cost-effective compared with fusion after 2 years, ICER $=€ 126,300 / \mathrm{QALY}$.

Conclusions: When introducing new surgical techniques, the gains must be weighed against the cost. An RCT should be conducted with a health economic perspective, to make a better ground for decisionmaking. These three examples show that the assessment of cost-effectiveness can help health providers to make priorities between treatments. Disclosures: Author 1: none; Author 2: none; Author 3: none; Author 4: none; Author 5: none.

\section{QF43}

\section{A SIMPLE WAY TO SEE THE SCOLIOSIS IN 3D: THE VERTEBRA VECTORS PROJECTION IN HORIZONTAL PLANE}

Tamas Illés, Fabien Lauer, Francois Lavaste, Jean Dubousset

Deptartment of Orthopedics, CHU-Brugman, ULB/VUB, Brussels, Belgium

There is no enough information about the horizontal plane appearance of scoliosis despite its 3D nature. A new concept, the posterior-anterior vertebral vector is proposed to facilitate the visualization and the understanding of deformation, especially in the horizontal plane. The aim of this study is to highlight the importance of information obtained from these vertebra vector-based top-view images in clinical practice.

Materials and methods: 136 patients without spine deformity and 824 patients with scoliosis ( 719 without surgery and 95 cases before 
and after surgery) underwent in standing position simultaneous AP and lateral X-rays using the EOS 2D/3D system. In each case 3D surface reconstructions were carried out by sterEOS 3D software, which served as a basis for the generation of the vertebra vectors. The vector's starting points are in the middle of the interpedicular segment, running parallel to the upper plate, ending on the line joining the anterior end plates points, defining thus the posteroanterior axis of the vertebrae. Representing the vector's projection on each plan is possible, we will focus only on its projection on the horizontal plane. Results: For normal spine, the vector projections in the horizontal plane are aligned with the posteroanterior anatomical axis. It is a natural phenomenon when the vertebrae don't have an axial rotation. The top view analysis of scoliosis is essential, because, despite the similar images in the frontal and sagittal planes, the horizontal plane occurrence of curves can be completely different. For scoliotic spine, the vectors projections in the horizontal plane provide information about the frontal curves, the lateral decompensation of the spine, the lateral displacement of the vertebrae from the posteroanterior anatomical axis. The direction and the deviation of vertebra vectors from the posteroanterior anatomical axis can learn about the axial rotation of the vertebrae. The vertebra vectors provide the informations on the impact of surgical treatment: diminution of the curves and the decompensation in the frontal plane, harmony of curves in the sagittal plane, reduction of lateral deviations, and axial rotations in the horizontal plane.

Discussion: In the horizontal plane view all 3D component of scoliosis curves can be analyzed simultaneously. Projections of vertebra vectors in other axes may supplement this information, but some projections are overabundant. The only, horizontal representation with the vertebral vectors provides access to visualization for the better understanding of scoliosis and gives useful information in daily clinical practice before and after surgical treatment. The approach used is simple. These results are sufficient for a first visual analysis requires significant clinical information in the three anatomical planes. This visualization represents a reasonable compromise between mathematical purity and practical use.

Disclosures: Author 1: none; Author 2: none; Author 3: none; Author 4: none.

\section{QF44 \\ THREE-DIMENSIONAL RECONSTRUCTIONS OF THE RIB CAGE IN ADOLESCENT IDIOPATHIC SCOLIOSIS: WHAT DETERMINES GIBBOSITY?}

Ayman Assi, Mohammad Karam, Michel Salameh, Gerard Bakhos, Aya Karam, Georges Mjaess, Rhea Nacouzi, Eddy Saad, Naji Bou Zeid, Gaby Kreichati, Khalil Kharrat, Joeffroy Otayek, Anthony Kassab, Cyril Hanna, Chris Labaki, Joe Ghanimeh, Ziad Bakouny, Wafa Skalli, Ismat Ghanem

${ }^{1}$ Laboratory of Biomechanics and Medical Imaging, Faculty of Medicine, University of Saint-Joseph, Beirut, Lebanon; ${ }^{2}$ Institut de Biomécanique Humaine Georges Charpak, Arts et Métiers, ParisTech, Paris, France

Introduction: Subjects with adolescent idiopathic scoliosis (AIS) often seek treatment with the aim of correcting the gibbosity of the rib cage. Current surgical treatment for AIS mainly focuses on the correction of the spinal deformity. However, it is not yet known how the gibbosity is related to the deformity of the spine in $3 \mathrm{D}$.

Purpose: To quantify the relationship between the gibbosity and the 3D deformity of the scoliotic spine.

Methods: 74 patients with AIS (62F, age: $15 \pm 2$ years, Cobb: mean $=32^{\circ}\left(20^{\circ}-50^{\circ}\right)$ age-matched to 44 control subjects $(24 \mathrm{~F})$ underwent full-body biplanar X-rays with 3D reconstructions of the spine and the rib cage. The following parameters were collected for the rib cage: maximum thickness, maximum width, gibbosity, rib cage volume, volumetric spinal penetration index [2]. Sagittal spino-pelvic parameters (pelvic tilt, pelvic incidence, sacral slope, thoracic kyphosis, lumbar lordosis) were assessed as well as the frontal Cobb angle, and the axial rotation of the apical vertebra (AVR). A subset of the initial sample consisting of 30 subjects with AIS age-matched to ten control subjects were considered for the repeatability assessment and had their rib cages reconstructed three times by six trained operators. The reliability was assessed on this subset using the Intraclass Correlation Coefficients (ICC). The 95\% confidence interval (CI), including both intra and inter-observer variability, was calculated for each parameter (confidence in the estimate $<10 \%$ ). Comparison of gibbosity between the scoliotic and control groups was assessed (Mann-Whitney's test) on the whole sample. Factors associated to the gibbosity were identified using a stepwise multiple linear regression (SMLR) model.

Results: The reliability analysis of the 3D rib cage parameters showed an ICC $>0.98$. The $95 \%$ CI of the gibbosity varied between $2.2^{\circ}$ and $3.2^{\circ}$. The absolute value of gibbosity was significantly larger in the scoliotic group $\left(6^{\circ} \pm 4^{\circ}\right.$ vs $\left.3^{\circ} \pm 2^{\circ}, \quad p<0.001\right)$. Significant correlations $(\mathrm{p}<0.001)$ were found between gibbosity and both the coronal Cobb angle $(r=0.41)$ and the apical vertebral rotation $(r=0.6)$. Gibbosity was not associated to any of the sagittal spino-pelvic parameters. However, the SMLR model revealed that apical vertebral rotation was a significant determinant factor of gibbosity $(R 2=0.36, p<0.001$, Fig. 1). 64\% of the variability of gibbosity remained unexplained.

Conclusion: 3D reconstructions of the rib cage, based on biplanar $\mathrm{X}$-rays, were shown to be repeatable in both control and AIS subjects. This is the first study to investigate the relationship between the deformity of the rib cage and the one from the spine in subjects with AIS. AVR was shown to be the most determinant factor of gibbosity. Other unexplored factors, probably related to the mechanical properties of the ribs and soft tissues, could also be implicated in gibbosity generation.

\section{References}

[1] Aubert B, CMBBE, 2016. [2] Dubousset J, Orthop Sci, 2003.

Disclosures: Author 1: none; Author 2: none; Author 3: none; Author 4: none; Author 5: none; Author 6: none; Author 7: none; Author 8: none; Author 9: none; Author 10: none.
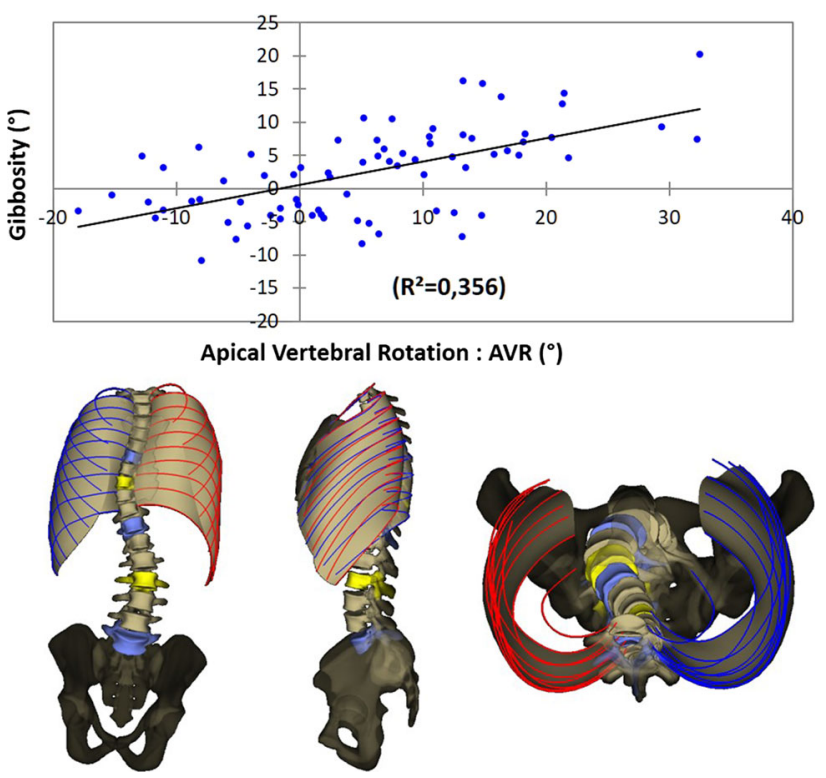


\section{QF45}

\section{WHAT DETERMINES ERRORS ON 3D SPINE RECONSTRUCTIONS OF SCOLIOTIC PATIENTS?}

Joeffroy Otayek, Ayman Assi, Gerard Bakhos, Cyril Hanna, Ziad Bakouny, Mohammad Karam, Wafa Skalli, Fares Yared, Aren Joe Bizdikian, Ismat Ghanem

${ }^{1}$ Laboratory of Biomechanics and Medical Imaging, Faculty of Medicine, University of Saint-Joseph, Beirut, Lebanon; ${ }^{2}$ Institut de Biomécanique Humaine Georges Charpak, Arts et Métiers ParisTech, Paris, France

Introduction: The coronal Cobb angle is the most common method to assess the severity of a scoliotic curve. With recent technology, it is now possible to measure the Cobb angle in $3 \mathrm{D}$ as well as many other parameters such as the apical vertebral rotation (AVR) and sagittal curvatures using the $3 \mathrm{D}$ reconstructions obtained from biplanar radiographs. While the reliability of Cobb angle measurement has been largely studied in the literature, measurements were mainly $2 \mathrm{D}$ and performed based on pre-determined end verterbrae [1]. However, in clinical routine, physicians choose the end vertebrae subjectively, leading to possible differences in Cobb angle measurements between physicians.

Purpose: To quantify and determine the errors and their causes on 3D Cobb angle and AVR measurement in patients with Adolescent Idiopathic Scoliosis (AIS).

Methods: 67 patients with AIS (59F, age: $14 \pm 3$ years, Cobb: $10^{\circ}-$ $106^{\circ}$ ) underwent full body biplanar X-rays and were included in this study. Both major and minor curvatures were considered (94 curvatures in total), and stratified by Cobb severity as: $\operatorname{Gr} 1:\left(10^{\circ}-20^{\circ}\right)$ $\mathrm{N}=18, \mathrm{Gr} 2:\left(20^{\circ}-40^{\circ}\right) \mathrm{N}=30, \mathrm{Gr} 3:\left(40^{\circ}-70^{\circ}\right) \mathrm{N}=30, \mathrm{Gr} 4:>70^{\circ}$ $\mathrm{N}=16$. Three trained operators performed $3 \mathrm{D}$ reconstructions of the spine three times each. Operators were asked to identify the endvertebrae by choosing the most tilted vertebrae in the coronal plane. Collected parameters were: end-vertebrae levels, coronal Cobb angle, sagittal curvatures (TK, LL), apical vertebral rotation (AVR) and orientation of the end-vertebrae in the three planes (Fig. 1a). Reliability on the levels of the end-vertebrae, Cobb angle and AVR were evaluated by calculating the reproducibility coefficient (SR) (ISO 5725-2). Stepwise multiple linear regressions (SMLR) were used in order to identify the sources of error for each of the parameters.

Results: The SR ranged from 0.4 to 0.9 levels for the end vertebra level (Fig. 1b), from $2.9^{\circ}$ to $3.8^{\circ}$ for the 3D Cobb angle (Fig. 1c) and from $2.8^{\circ}$ to $6.2^{\circ}$ for the AVR angle (Figure 1d). SMLR revealed that the uncertainty on the choice of the end-vertebrae was determined by the severity of the Cobb angle $(\beta=-0.235, p=0.001)$. The uncertainty on the Cobb angle was determined by the severity of the Cobb angle $(\beta=0.382, p<0.001)$ and by the uncertainty on end-vertebrae $(\beta=0.221, p=0.035)$. Uncertainty on AVR was determined by the Cobb angle $(\beta=0.444, p<0.001)$, T4-T12 kyphosis $(\beta=0.404$, $\mathrm{p}<0.001)$ and the value of the AVR $(\beta=-0.211, \mathrm{p}=0.023)$.

Conclusions: The uncertainties on $3 \mathrm{D}$ Cobb angle and AVR measurement were found to be predominantly determined by increasing Cobb angle value, possibly due to increased difficulty in visualization of vertebral anatomical landmarks with increasing scoliosis severity. Further standardization of end vertebrae selection methods could therefore improve 3D Cobb angle measurement, in particular for smaller curvatures.

\section{References}

[1] Ilharreborde B, et al., Spine, 2011.

Disclosures: Author 1: none; Author 2: none; Author 3: none; Author 4: none; Author 5: none; Author 6: none; Author 7: none; Author 8: none; Author 9: none; Author 10: none.

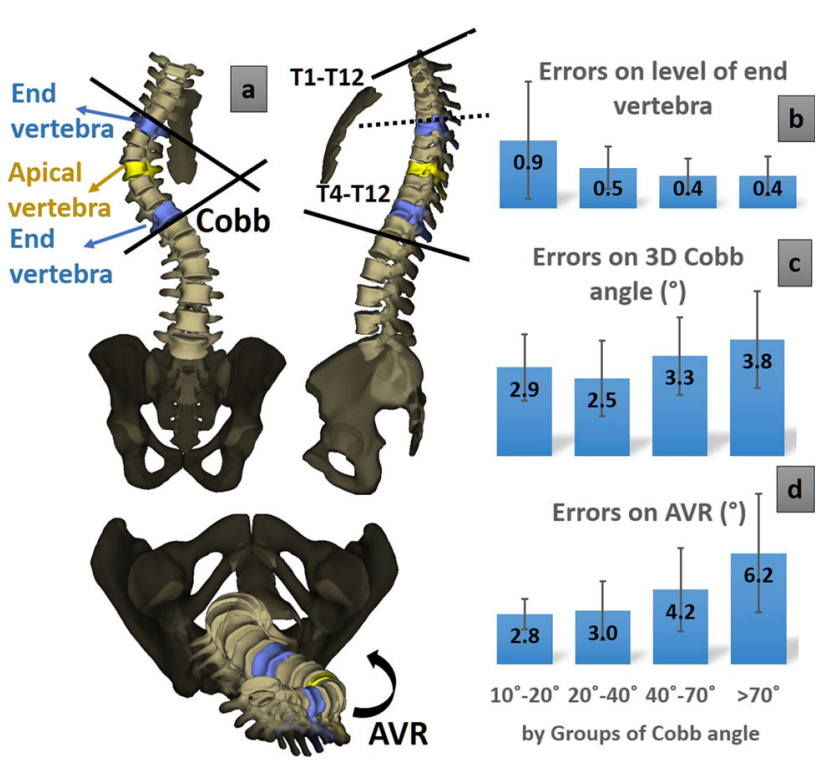

QF46
THE USE OF PATIENT SPECIFIC TEMPLATES IN
THORACIC SCOLIOSIS SURGERY

Janez Mohar, Robert Cirman, Rene Mihalic

Department of Spine Surgery and Paediatric Orthopaedics, Valdoltra Orthopaedic Hospital, Ankaran, Slovenia

Introduction: Pedicle screw based instrumentation in thoracic scoliosis surgery offers superior fixation strength, improved deformity correction and increased bone fusion rate as opposed to constructs with other anchoring implants. Despite being a gold-standard in spine deformity surgery, thoracic pedicle screw placement can, by either intraoperative image-assisted or free-hand cannulation techniques, sometimes be inaccurate, resulting in variety of implant related complications, i.e., nervous, pulmonary or vascular injury.

The literature data on usability of additive manufacturing technology in spine surgery is scarce.

Purpose of the study: Primarily, we aimed to determine the concordance of actual thoracic pedicle screw positions with the preoperative planning in thoracic scoliosis patients in which pedicle screw placements were performed with the use of patient specific rapid prototyping 3D templates as the pedicle screw entry point and trajectory guides. Secondarily, we wanted to asses the accuracy of pedicle screw placements by this novel technique and its usability in thoracic deformity surgery.

We hypothesised that actual thoracic pedicle screw positions will be in high accordance with the preoperative 3D planning.

Materials and methods: Twenty-one consecutive scoliosis patients (17 idiopathic, 3 neuromuscular, 1 congenital) underwent posterioronly pedicle screw fixation and deformity correction surgery amounting to 147 thoracic pedicle screw placements performed with the use of patient specific $3 \mathrm{D}$ templates.

On CT scans each postoperative screw position, defined by the entry point and two trajectory angles on oblique sagittal and oblique transverse plane, was compared to its preoperative planed position. For each instrumented pedicle a Zdichavsky pedicle breach grade was 
evaluated and suboptimal screw positions were further defined by Gertzbien and Heary cortical breach classifications.

Results: Compared to the preoperative planning, the average instrumented screw entry point offset on $\mathrm{X}$ and $\mathrm{Y}$ axes was $0.7 \mathrm{~mm}$ and $0.8 \mathrm{~mm}$, respectively. The average difference in trajectory between the planned and the actual screw on the oblique sagittal and the oblique transverse plane was $2.3^{\circ}$ and $2.2^{\circ}$, respectively. According to the Zdichavsky classification, 140 screws were grade Ia (optimal placement), two screws were grade IIa, four screws were grade IIb and one screw was grade IIIa.

Conclusion: The predictive value of preoperative plans in our case series is $95 \%$, which is also the absolute accuracy of pedicle screw placements with the use of patient specific 3D templates. Considering the previously reported "safe zone" of medial and lateral pedicle breaches of 2 and $4 \mathrm{~mm}$, respectively, the relative accuracy of this technique is $97 \%$, which by far exceeds the accuracies of pedicle screw placements in deformed thoracic spine by fluoroscopy guided, navigated or free-hand techniques.

Disclosures: Author 1: none; Author 2: none; Author 3: consultant: Medacta International SA, Switzerland.

\section{QF47 \\ COMPARISON BETWEEN DIFFERENT RADIOGRAPHIC METHODS FOR ASSESSMENT OF THE CURVE FLEXIBILITY IN AIS ( $>70^{\circ}$ CURVES)}

Sinan Kahraman, Selhan Karadereler, Cem Sever, Isik Karalok, Sezgi Burcin Barlas, Gokce Feride Inan, Tunay Sanli, Meric Enercan, Azmi Hamzaoglu

\section{Department of Orhopaedics and Traumatology, Istanbul, Turkey}

Summary: Traction X-ray under general anesthesia (TrUGA) shows better flexibility in main thoracic and thoracolumbar/lumbar curves $>70^{\circ}$ when compared with Fulcrum, Bending and traditional traction $\mathrm{X}$-rays. TRUGA also shows a better correlation with postoperative radiologic results.

Hypothesis: TrUGA offers better flexibility and predicts postoperative radiologic results than other radiographic methods. General anesthesia enables absolute muscle relaxation and eliminates patient cooperation and technician factors.

Introduction: In this study we aimed to compare the corrective ability and predictability of Traction X-ray under general anesthesia (TRUGA) with Bending (BXR), Fulcrum (F) and Traditional traction (TTr) X-rays in AIS patients with $>70^{\circ}$ curves.

Methods: A total of $126(105 \mathrm{~F}, 21 \mathrm{M})$ consecutive patients with AIS who had $>70^{\circ}$ MT or TL/L curves and who had surgical treatment were studied. Preop radiologic evaluation consisted of standing AP/ LAT, BXR, F, TTr and TRUGA just before surgery. All curves were measured, and the flexibility ratio was determined on each radiograph. The amount of correction obtained by all radiographic methods was compared with the amount of surgical correction by evaluating the differences from surgery as absolute values. Mean absolute differences from surgery were used to determine the confidence intervals. Statistical differences were calculated with Friedman and Wilcoxon signed rank test.

Results: The MT curve was $>70^{\circ}$ in 69 patients $(54.8 \%)$, TL/L curve was $>70^{\circ}$ in 38 patients $(30.2 \%)$ and both curves were $>70^{\circ}$ in 19 patients $(15 \%)$. Average preop Cobb angle for MT and TL/L curves were $76^{\circ}(70-114)$ and $72^{\circ}(70-77)$, respectively. For both MT and $\mathrm{TL} / \mathrm{L}$ curve $>70^{\circ}$ group preop cobb angles were $95^{\circ}(70-130)$ and $77^{\circ}$
(70-94), respectively. For MT curves, TRUGA provided clearly better flexibility compared to BXR, F and TTr X-rays and gives most close value to postop $\mathrm{X}$-ray.(Table 1$)(\mathrm{P}<0.05)$. TRUGA and BXR predicts better flexibility for TL/L curves and correlation with post op correction than the other X-rays $(\mathrm{P}<0.05)$. But there is no statistically significance for TL/L curve flexibility between TrUGA and BXR ( $>0.05)$.

Conclusion: TrUGA demonstrated highest flexibility rate for all curves $>70^{\circ}$ in AIS and gives better prediction with post op radiologic results. This study showed us that the TrUGA, taken in addition to the standing X-ray, gives more detailed information in the determination of flexibility and estimation of postoperative correction, therefore there is no need to take bending, fulcrum, and traditional traction $\mathrm{X}$-rays. The application of this principle will help to eliminate the excessive radiation exposure of the patients.

Disclosures: Author 1: none; Author 2: none; Author 3: none; Author 4: none; Author 5: none; Author 6: none; Author 7: none; Author 8: none; Author 9: consultant: Medtronic.

\begin{tabular}{|c|c|c|c|c|c|c|c|}
\hline $\mathrm{N}=126$ Mean (min-max & & PREOP & BENDING & FULCRUM & TRACTION & TRUGA & FOLLOW/UP \\
\hline$M T>70^{\circ}\left({ }^{\circ}\right)(n=69)$ & & $76(70-114)$ & $49,2(23-97)$ & $53,1(18-92)$ & $61,8(38-94)$ & $28,8(15-74)$ & $18,9(2-62)$ \\
\hline $\mathrm{TL} / \mathrm{L}>70^{\circ}\left(^{\circ}\right)(\mathrm{n}=38)$ & & $72(70-77)$ & $29,3(18-66)$ & $41,2(24-68)$ & $59,1(38-73)$ & $27,9(18-40)$ & $16,3(12-30)$ \\
\hline MT \& TL/L $>70^{\circ}$ & MT & $95(70-130)$ & $60,4(38-94)$ & $62,6(36-78)$ & $73,2(44-94)$ & $35,2(22-64)$ & $28,1(14-40)$ \\
\hline$\left(^{\circ}\right)(n=19)$ & $T L / L$ & $77(70-94)$ & $29,8(21-64)$ & $42,8(24-68)$ & $59,4(39-70)$ & $26,4(17-43)$ & $17,3(10-31)$ \\
\hline
\end{tabular}

\section{QF48 \\ FAILURE TO VALIDATE THE AGE-ADJUSTED ALIGNMENT THRESHOLDS CONCEPT IN AN ADULT SPINAL DEFORMITY DATABASE}

Caglar Yilgor, Nuray Sogunmez, Yasemin Yavuz, Ibrahim Obeid, Frank S. Kleinstueck, Francisco Javier Sanchez Perez-Grueso, Emre R. Acaroglu, Ferran Pellisé, Ahmet Alanay, ESSG European Spine Study Group

School of Medicine, Acibadem University, Istanbul, Turkey

Background: Schwab's sagittal modifiers have been accepted as alignment targets but addressing these does not always prevent mechanical complications. Spinopelvic alignment is known to vary for age. Attempts have been made to adapt these changes to ASD realignment objectives. Formulae were proposed to calculate ageadjusted alignment thresholds. PT is defined as $($ Age -55$) / 3+20$. PI-LL is $($ Age -55$) / 2+3$. SVA is $2 \times($ Age -55$)+25$. Thresholds for over and undercorrection was based on patient age \pm 10 years.

Purpose: The aim of this study was to validate age-adjusted Schwab alignment thresholds on both HRQoL scores and mechanical complication rates.

Methods: This study is a retrospective analysis of a prospectively collected multicentric data of ASD patients. Inclusion criteria were $\geq 4$ levels fusion and $\geq 2 y$ follow up. 222 patients (168 female, 54 male) met the inclusion criteria. Mean age was $52.2 \pm 19.3$ (18-84) years. Mean follow up was $28.8 \pm 8.2$ (24-62) months. ODI, COMI, SF-36 PCS, MCS and SRS-22 pain, function, mental health, self image and subtotal scores were used as patient-reported Health Quality of Life (HRQoL) scores. PJK/PJF, DJK/DJF, rod breakage and implant-related complications were recorded as mechanical complications. Patients were classified as undercorrected, matched or overcorrected according to the proposed formulae. The Chi Squared test was performed to compare mechanical complication rates for PT, PI-LL and SVA groups and age-adjusted groups. Last follow up 
HRQoL scores were compared using ANCOVA eliminating the effect of pre-op HRQoL scores.

Results: Mechanical complication rates were similar for PT matched and undercorrected ( $\mathrm{p}>0.05)$, for PI-LL matched and overcorrected $(\mathrm{p}>0.05)$ and for all age-adjusted groups in SVA $(\mathrm{p}>0.05)$. ODI, SF-36 PCS and MCS were not different in age-adjusted groups $(\mathrm{p}=0.055, \mathrm{p}=0.516$ and $\mathrm{p}=0.381$, respectively). SRS22 subtotal score was different in age-adjusted groups $(p=0.019)$ with undercorrected group having the best results.

Conclusion: This study in an independent adult spinal deformity (ASD) database failed to validate the age-adjustment concept that proposes to target ideal sagittal alignment goals according to age. Reaching age-adjusted Schwab realignment goals in ASD surgery failed to improve, if not worsened, clinical outcomes and to prevent mechanical complications.

Disclosures: Author 1: none; Author 2: grants/research support: DePuy Synthes; Author 3: none; Author 4: grants/research support: DEPUY SYNTHES, consultant: MEDTRONIC, DEPUY SYNTHES, royalties: ALPHATEC, SPINEART, CLARIANCE; Author 5: grants/ research support: Depuy-Synthes; Author 6: grants/research support: DEPUY SINTHES, consultant: K2M. DEPUY SINTHES; Author 7: grants/research support: Depuy Synthes, Medtronic, stock/shareholder: IncredX, royalties: Zimmer Biomet, AOSpine; Author 8: grants/research support: DePuy Synthes Spine, Medtronic, consultant: DePuy Synthes Spine, Biomet; Author 9: grants/research support: Depuy Synthes, consultant: Medtronic; Author 10: grants/research support: DePuy-synthes.
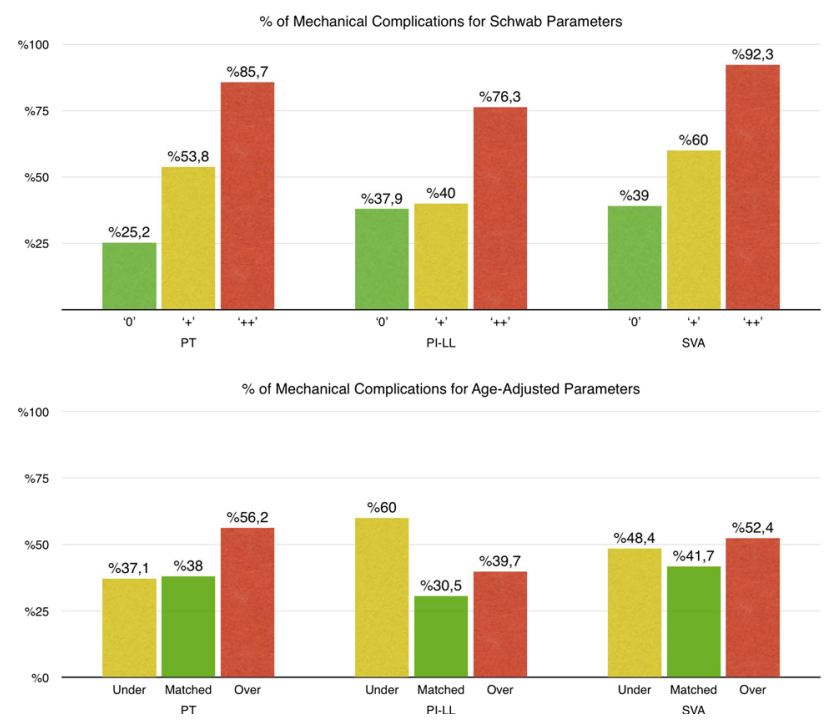

\section{QF49 \\ ARE PATIENT-SPECIFIC RODS EFFECTIVE TO MANAGE ADULT SPINAL DEFORMITY?}

Vincent Fiere, Henri D’Astorg, Marc Szadkowski, Evalina Burger

Spine Unit, Santy Orthopaedic Center, Lyon, France

Introduction: Despite advanced instrumentations for adult deformity management and proven correlations between health-related quality of life data and sagittal alignment, a significant proportion of patients remain under-corrected or malaligned after surgery. Recent development of patient-specific spinal technology is available to improve radiological outcomes. Since September 2013, patient-specific rods were implanted in more than 120 patients at a single center with the primary goal of reaching a preoperatively-defined patient-specific sagittal alignment in radiological correction.

Hypothesis: Patient-specific rods in association with preoperative surgical planning are likely to improve radiological outcomes in adult spinal deformity.

Design: Retrospective review of a consecutive prospective study.

Methods: Patients-specific rods are designed from preoperative imaging following the patient's anatomy and spinal correction required. Patients-specific rods were implanted in more than 120 patients in our center among whom, 60 achieved 1 year follow-up and 33 achieved 2 years follow-up. Patients malaligned at baseline for at least one SRS-Schwab parameter [either pelvic tilt (PT), pelvic incidence-lumbar lordosis mismatch (PI-LL), sagittal vertebral axis (SVA) or Cobb Angle], planned within the threshold for all of these parameters combined, implanted with patient-specific rods and reaching 1-year post-operative follow-up with full spine imaging were included producing a cohort of 34 patients with a mean age of 64.4 years, $65 \%$ women, $23 \%$ receiving pedicle subtraction osteotomies (PSO) and a mean of 7 levels instrumented.

Results: Compared to published data with conventional treatment, patients receiving patient-specific rods were found to be 2.6 times more likely to be corrected in the sagittal plane for all parameters combined and two times more likely to be corrected for PT, the most challenging criteria to manage. Patients who underwent PSO were found to be 3.8 times more likely to be corrected in the sagittal plane with all parameters improved at 1-year post-op. Further, the custom implants avoid intraoperative rod bending and are expected to reduce surgical time and prevent rod-notching, a known contributor to breakage. To date, no incidence of rod breakage was found in the whole cohort $(\mathrm{n}=120)$.

Conclusion: This study confirms the relevance of a personalized spinal surgery to achieve sagittal alignment. Radiological outcomes achieved through preoperatively-defined patient-specific rods are promising and need to be confirmed at longer follow-up and correlated with clinical outcomes.

Disclosures: Author 1: consultant: Medicrea, Clariance, royalties: Medicrea, Clariance; Author 2: consultant: Clariance; Author 4: grants/research support: 4. Activ-L Artificial Disc. CT, Aesculap, Inc., Industry, 11/28/2007 to 6/30/2016, \$1448,783, consultant: DSM, Paradigm Spine, Signus, X-Spine, Medicrea, Medacta, Spine Wave.

\section{QF50 \\ USE OF S2-ALAR-ILIAC SCREWS DOES NOT PROMOTE SACROILIAC JOINT FUSION IN PATIENT WITH ADULT SPINAL DEFORMITY}

Zhen Liu, Yong Qiu, Changchun Tseng, Bangping Qian, Bin Wang, Zezhang Zhu

Spine Surgery, Drum Tower Hospital of Nanjing University Medical School, Nanjing, China

Background: S2-Alar-Iliac (S2AI) screw is an alternative technique to the iliac screw in patient with adult spinal deformity. Spinopelvic fixation utilizing S2AI has some advantages over the others including no offset modular connector required, less dissection and less instrumentation prominence. However, one concern to this technique is associated with the fact that the S2AI screw starts from the sacrum and is placed across the sacroiliac joint and into the ilium. This trajectory may affects outcomes because of sacroiliac joint arthritis, pain, degeneration or sacroiliac joint-related dysfunction. There is no study evaluating both the sacroiliac joint fusion and sacroiliac joint 
related health quality related of life after spinopelvic fixation utilizing S2AI technique.

Objective: This study was designed to evaluate whether there was sacroiliac joint fusion and health-related quality of life after pelvic fixation utilizing S2AI.

Methods: This study was approved by our Institutional Review Board. We retrospectively review all patients who underwent placement of S2AI screws at our institution between Jan 2014 and Jan 2016. 16 patients underwent spinal fusion with S2AI pelvic fixation who had complete radiographic data including Computed Tomography scan of sacroiliac joint were enrolled in this study. All patients have received CT scans preoperatively and at last follow-up. CT scans were reviewed to determine whether there was sacroiliac joint fusion. According to the previous study, we chose and applied five tests with high sensitivity and specificity to diagnose sacroiliac joint dysfunction. These tests were administered to all patient at the last follow-up. Three tests resulting positive was regarded as dysfunction. The tests applied were as follows: The Gillet Test, Standing Flexion Test, Distraction Test, Gaenslen's Test and Sacral Thrust Test.

Results: Sixteen patients were enrolled (mean age, $50.25 \pm 17.05$ years), ten of them are female. The mean Cobb angle was $36.35^{\circ}$ (SD $8.97^{\circ}$ ) preoperatively and $16.75^{\circ}$ (SD 9.24 $)$ postoperatively. The follow-up period ranges from 12 to 28 months. There were no instances of osseous fusion across the sacroiliac joint on the last follow-up CT (figure 1). All patients resluted negative in the physical exam.

Conclusion: S2AI seems to be a superior spinopelvic fixation technique with minor adverse effect on daily life based on our retrospective study. During the follow-up period ranging from 12 to 28 months in our study, sacroiliac joint remains normal function and no observation of osseous fusion across this joint.

Disclosures: Author 1: none; Author 2: none; Author 3: none; Author 4: none; Author 5: none.

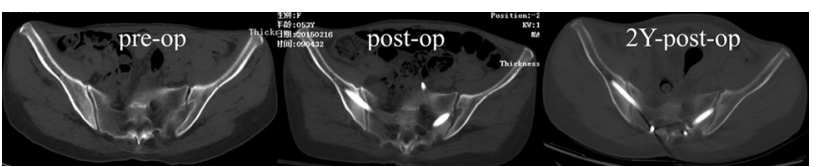

\section{QF51 \\ ALTERATION OF SENSORIMOTOR NETWORK IN LUMBAR SPINAL STENOSIS USING RESTING-STATE FUNCTIONAL CONNECTIVITY MRI}

Eiji Takasawa, Yasunori Sorimachi, Yoichi Iizuka, Haku Iizuka, Hirotaka Chikuda

Department of Orthopaedic Surgery, Japanese Red Cross Maebashi Hospital, Maebashi, Japan; Department of Orthopaedic Surgery, Gunma University Graduate School of Medicine, Maebashi, Japan

Introduction: Lumbar spinal canal stenosis (LSS) is very common among older persons and may lead to walking disabilities due to motor and equilibrium disturbances. The conventional diagnosis of LSS relies on neurological examinations as well as anatomical imaging of spinal local lesions (e.g. X-ray, CT scanning, or MRI). Recently, novel neuroimaging techniques have revealed compensatory neural changes in the brain during neurological recovery of spinal disorders, such as spinal cord injury and scoliosis. However, there is little information about the alterations to neural functions of the central nervous system in patients with LSS. Resting-state functional connectivity MRI (rsfc-MRI) is a new type of functional MRI
(fMRI) that allows for the noninvasive visualization and measurement of neural signals, as well as for the assessment of "brain networks" during rest. The purpose of this study is to using rsfc-MRI to explore how the brain neural network is altered in patients with LSS.

Methods: We enrolled 5 patients with LSS (LSS group) and 5 healthy volunteers (control). fMRI data were acquired on a 3-T MRI scanner during rest with the subject's eyes open. To reveal the specific neural networks affected by LSS, the data were preprocessed and computed using CONN software (NITRC, funded by NIH). Among the various resting state networks, we focused on the sensorimotor network (SMN): this network is involved in preparing the brain to perform and co-ordinate a motor task. To detect functional changes to the SMN in patients with LSS, we compared the pattern and strength of SMN connectivity between the two groups.

Results: In the LSS group, stronger functional connectivity was observed in the primary motor cortex (M1), the primary sensory cortex (S1), and the supplementary motor cortex, which constitute the SMN. Moreover, stronger functional connectivity was observed between the M1 and the cuneus, a part of the occipital lobe that is involved in visual processing. In contrast, LSS was associated with lower functional connectivity between the M1 and the cerebellum, which plays an important role in motor control, equilibrium, and posture.

Conclusion: Using rsfc-MRI, we reveal the intra- and inter-network alterations to the SMN in patients with LSS. Our results suggest that the increased intrinsic connectivity of the SMN and the complementary inter-networking between the motor and visual cortices may be a compensatory response for motor and equilibrium disturbances in LSS. These findings could provide clinical information to promote an understanding of the dynamicity of the brain neural network and to develop neurological rehabilitation strategies for patients with LSS. Disclosures: Author 1: none; Author 2: none; Author 3: none; Author 4: none; Author 5: none.

\section{QF52 \\ TREATMENT OF CHRONIC LOW BACK PAIN VIA ABLATION OF THE BASIVERTEBRAL NERVE: RESULTS OF THE SMART TRIAL}

Jörg Franke, Bernhard Meyer, Peter Vajkoczy, Alfred Rhyne, Jeff Fishground, Rick Sasso, Hyun Bae, Christopher Yeung, Eeric Truumees, Philip Yuan

Department of Orthopedics; Klinikum Magdeburg, Magdeburg, Germany

Introduction: The basivertebral nerve $(\mathrm{BVN})$ is a sensory nerve within the vertebral body, whose role in pain transmission is thought to be a source of chronic low back pain associated with degenerative changes. The use of a radiofrequency system (INTRACEPT ${ }^{\mathrm{TM}}$ System) for ablation of the BVN was tested in this trial.

Methods: 225 patients with chronic low back pain refractory to $>6$ months of conservative care and no neurogenic leg pain were enrolled at 18 sites in the United States and Germany in this prospective, double blinded, randomized, sham controlled trial (147 active vs 78 sham arm). MRI had to demonstrate Type 1 or 2 Modic changes at $<3$ contiguous vertebral bodies. Follow-up was at 2 and 6 weeks, 3, 6, and 12 months. Patients and physicians providing postoperative care were blinded to allocation until the conclusion of the 1 year follow-up. For sham patients, there was an optional crossover after all 12-month evaluations.

Results: Targeting success (assessed by postop MRI) was achieved in $96.4 \%$ of the treated and evaluated vertebral bodies. Primary end 
point analysis in the per protocol population at 3 months showed that the ODI improvement in the active arm was superior to the sham arm ( $\mathrm{p}=0.019)$ (mean improvement: 20.5 points). This result was sustained through 2 years of follow-up. An analysis of ODI responder rates found that $75.6 \%$ of the RF ablation treated patients demonstrated a greater than 10-point, clinically meaningful improvement in their low back pain and associated disability at 3 months. There were no adverse device effects and no device related serious adverse events. The rates of neurological events reported were low and comparable between treatment arms.

Conclusions: BVN ablation with the INTRACEPT ${ }^{\mathrm{TM}}$ System proved to be safe and effective for the treatment of chronic low back pain in this patient population based on the primary end point results showing significantly greater improvement in ODI for the active arm.

Disclosures: Author 1: grants/research support: Baxter; medtronic, Paradigm, relievant, consultant: medacta, Medtronic, zimmer, Paradigm, safe orthopedics, Eos imaging, baxter, EIT, royalties: Medtronic, OHST, medacta; Author 2: grants/research support: Relievant, Medtronic, Icotec, Ulrich, consultant: Relievant, Medtronic, Icotec, Ulrich, royalties: Spineart; Author 4: none; Author 5: consultant: Stryker and Relievant, royalties: Stryker; Author 6: grants/ research support: Medtronic, royalties: Medtronic; Author 8: consultant: Advance Biologics, Globus Medical, Lattice Biologics Ltd, Paradigm Spine, Signus Medical, Stryker Spine, stock/shareholder: Bonovo Orthopedics, Lattice Biologics, Paradigm Spine, Mobius, Plasmology 4, royalties: Globus Medical, Signus Medical, employee: Desert Institute for Spine Care, co-owner, Squaw Peak Surgical Facility, co-owner, Arizona Recovery Care Center, co-owner, The CORE Institute Specialty Hospital (formerly known as The Surgical Hospital of Phoenix, ) - part owner, Surgical MRI, co-owner; Author 9: grants/research support: Pfizer, royalties: Stryker; Author 10.

\section{QF53 \\ DYNAMIC POSTERIOR STABILIZATION VERSUS POSTERIOR LUMBAR INTERVERTEBRAL FUSION: A MATCHED COHORT STUDY BASED ON THE SPINE TANGO REGISTRY}

\section{Kelly Goodwin, Kathrin Bieri, Emin Aghayev, Ralph Greiner-Perth}

SwissRDL, Institute of Social and Preventive Medicine, University of Bern, Bern, Switzerland

Purpose: The primary aim of dynamic stabilization is partial stabilization, while avoiding the negative side effects of fusion surgery like overstress of adjacent segments. However, comparative analyses of the two approaches are still limited, and little is known about association of patient-reported outcomes with the treatment options. The objectives of this study were to compare the clinical outcomes of dynamic posterior stabilization using DSS ${ }^{\circledR}$ with posterior lumbar intervertebral fusion (PLIF) based on data from a spine registry. We hypothesised that patient-reported outcomes of $\mathrm{DSS}^{\circledR}$ are not inferior to those of PLIF.

Methods: We identified 202 DSS $^{\circledR}$ and 323 PLIF patients with degenerative disease or spondylolisthesis involving up to three lumbar segments who were initially treated conservatively and later surgically with a minimum 2-year follow-up. Cases with more than two surgeries were excluded. A 1:1 propensity score-based matching was applied to balance the groups for various patient characteristics and test the non-inferiority hypothesis of DSS ${ }^{\circledR}$ in comparison to PLIF. The primary outcome was the change in patient-reported Core Outcome Measures Index (COMI; a 0-10 scale) score and the inferiority was set to 2 points corresponding to the minimum clinically important change. Secondary outcomes were back and leg pain relief, rates of repeat surgery, surgical and general complications, blood loss, surgery duration, and length of hospital stay. We calculated also the number of repeat surgeries per 100 observed person-years.

Results: The matching resulted in 81 DSS $^{\circledR}$-PLIF pairs (mean age 68 years; average COMI follow-up 3.4 years) without residual significant differences in baseline characteristics. The groups showed no difference in COMI score improvement $(\mathrm{p}=0.25)$, as well as in back $(\mathrm{p}=0.45)$ and leg pain relief $(\mathrm{p}=0.79)$, blood loss $(\mathrm{p}=0.11)$, and in complications $(p>0.25)$. However, less repeat surgeries after DSS $^{\circledR}$ than after PLIF were seen $(p=0.030)$. The number of repeat surgeries per 100 observed person-years was 1.2 and 3.0 in DSS ${ }^{\circledR}$ and in PLIF patients, respectively. Furthermore, shorter surgery time $(p<0.001)$, and longer hospital stay $(p=0.035)$ were observed for $\mathrm{DSS}^{\circledR}$ in comparison with PLIF cases.

Conclusion: In mid-term perspective, $\mathrm{DSS}^{\circledR}$ may be a good alternative to PLIF as both therapies result in similar COMI score improvement. Moreover, DSS ${ }^{\circledR}$ may be beneficial to PLIF in shorter surgery duration and less repeat surgeries. However, $60 \%$ of DSS patients did not find a matched pair implying that they were rather different from the eligible PLIF patients regarding the patient characteristics used for matching. Further multi-centre studies are needed to better understand the indication limits of the therapies.

Disclosures: Author 1: none; Author 2: none; Author 3: none; Author 4: none.

\section{QF54 \\ DOES TI-COATING IMPROVE PEEK CAGES OSTEOCONDUCTIVE PROPERTIES? A PROSPECTIVE RANDOMIZED CLINICAL TRIAL}

Marcus Rickert, Michael Rauschmann

Spine Department, University Hospital Friedrichsheim gGmbH, Frankfurt am Main, Germany

Introduction: Transforaminal lumbar interbody fusion (TLIF) is an established, safe technique for performing arthrodesis of the lumbar spine. Of the several different types of intervertebral cages used for TLIF currently on the market, studies have shown that those made of polyetheretherketone (PEEK) and titanium materials reliably produce successful fusion rates and good clinical outcomes. PEEK cages were introduced to overcome subsidence or delay fusion of Titanium cage, due to their stiffness and radiopacity limit for postoperative evaluation of fusion. However, despite these advantages, concerns have been raised about the use of PEEK because of its lack of osteoconductivity alongside its hydrophobicity which may negatively impact primary cage stability and the overall fusion rate. Titanium spray coating may circumvent these potential limitations by enhancing shear strength at the implant/bone interface. The aim of this study is to investigate whether titanium coating improves clinical or radiological outcome of TLIF PEEK cages, considered the gold standard material for such cages.

Patients and methods: The study was approved by Ethic Committee to enroll 40 patients, scheduled to undergo instrumented spinal arthrodesis with insertion of the implants in L2-L5. Patients were randomly allocated in PEEK and TiPEEK groups. Fusion rates were assessed by computed tomography at 3 months and by functional radiography at 12 months. Clinical and functional outcomes were assessed by the ODI and EuroQoL-5D scores, and VAS scale (for back and leg pain). The questionnaires were collected preoperatively, and at 3,6, and 12 months postoperatively. 
Results: Complete/partial solid fusion was shown by 21/1 TiPEEK levels vs 17/5 PEEK levels respectively through independent analysis of CTs at 3 months: $87.5 \%$ complete fusion rate for TiPEEK vs $70.8 \%$ for PEEK. Functional radiography at 12 months reveal indirect occurrence of fusion as the range of motion analysis shows comparable results between the two groups $\left(0.9^{\circ}\right.$ PEEK vs $0.6^{\circ}$ TiPEEK). Disc height preservation of the indexed segments $(88.8 \%$ PEEK vs $96.2 \%$ TiPEEK) or the adjacent segment $(85.7 \%$ PEEK vs $87.4 \%$ TiPEEK) show positive trend for the TiPEEK group. Both groups' functional, clinical and quality of life outcomes improved postoperatively.

Conclusion: Positive results were observed in both groups. TiPEEK cages showed favorable outcomes achieving even better fusion rates than PEEK cages. TiPEEK shows also a favorable result in term of disc height restoration and appears to potentially reduce the chance of subsidence after 1 year. Not only titanium coating appears to have no negative effects but positive outcome and safety in the short-medium term are seen. Additional studies with enlarged sample size are mandatory to better investigate clinical advantages of introducing a Ti-coating in term of statistical significance.

Disclosures: Author 1: grants/research support: Medacta, consultant: Medacta, Spontech; Author 2: grants/research support: Nanobone, 4web, paradigm spine, medacta.

\section{TRAUMA, TUMOR}

\section{QF55 \\ SURGICAL AGGRESSIVENESS INDEX IN SURGERY FOR METASTATIC SPINE DISEASE}

Naresh Kumar, Barry Tan, Aye Sandar Zaw

National University Health System, Singapore, Singapore

Introduction: Surgical management of spinal tumours utilizes a large spectrum of surgical techniques. In analyzing variables influencing surgical outcomes, comparing data between institutions, and weighing clinical options of treatment, a standardized scoring system that compares the extent of surgery with outcome variables will be useful for surgeons and patients alike in both research and treatment planning. We aimed to create a 'Surgical Aggressiveness Index' (SAI) in metastatic spine to classify surgical modalities employed that allows accurate prediction of operative parameters.

Methods: We devised a metastatic spine surgery scoring system based on surgical intrusion of the vertebral column. The score is based on number of vertebrae instrumented posteriorly or anteriorly, the number of levels decompressed by posterior approach, extent of anterior decompression dictated by total/partial corpectomy, posterior fusion and vertebroplasty.

The index was validated by the retrospective analysis of patients undergoing metastatic spinal surgery from 2005 to 2014. The relationship between the intrusion score and outcome measures such as blood loss, blood transfusion, operative time and post-operative complications were analyzed.

Results: A total of 241 patients were included in the analysis. The median age was 60 years (range 25-87) with gender distribution of 127 males $(53 \%)$ and 114 females (47\%). The median SAI was 7 (range 1-20). Multivariate analyses revealed that the Intrusiveness Index score was independently associated with increased amount of blood loss, increased odds of blood transfusion and prolonged operative time after adjusting for a number of potential confounders. With every one score increase in SAI, there was $42 \mathrm{ml}$ increase in mean blood loss (95\% CI 29-51, $\mathrm{P}=0.01), 10$ min increase in mean operative time (95\% CI 5-18, P $=0.01)$ and 1.1 times increased odds of blood transfusion (95\% CI 1.05-1.19, $\mathrm{P}=0.03$ ).

Conclusion: This novel SAI correlates well with surgical outcomes, in particular with blood loss, transfusion requirements and operative time. We believe that it will have significant utility in clinical practice for clinical decision-making and predicting risks and potential complications. In addition, this index would facilitate research by allowing fair comparisons between different types of operative procedures and operative outcomes between institutions.

Disclosures: Author 1: none; Author 2: none; Author 3: none.

Table 1

\begin{tabular}{ll}
\hline SAI modality & Score \\
\hline Posterior instrumentation per vertebral level & 1 \\
Anterior instrumentation per vertebral level & 1 \\
Posterior decompression Per Vertebral level & 1 \\
Partial corpectomy without cement/graft/cage per vertebral & 1 \\
$\quad$ level & \\
Partial corpectomy with cement/graft/cage per vertebral level & 2 \\
Total corpectomy per vertebral level & 4 \\
Posterior fusion per vertebral level & 1 \\
Vertebroplasty & 1
\end{tabular}

\section{QF56 \\ PROSPECTIVE EVALUATION OF MECHANICAL STABILITY IN RELATION TO PAIN RESPONSE TO RADIOTHERAPY FOR SPINAL METASTASES}

Joanne van der Velden, Anne Versteeg, Charles Fisher, Cumhur Öner, Jorrit-Jan Verlaan

Department of Radiation Oncology, University Medical Center Utrecht, Utrecht, The Netherlands

Introduction: A substantial number of patients with painful spinal metastases experience no effect of palliative radiotherapy. Besides tumor-induced pain, mechanical spinal instability due to metastatic disease, could be associated with failed pain control following radiotherapy. Early identification of patients who will not benefit from radiotherapy is important, since these patients might benefit more from surgical stabilization. This study aims to prospectively investigate the relation between spinal instability, as defined by the Spinal Instability Neoplastic Score (SINS), and the pain response to conventional palliative radiotherapy in patients with symptomatic spinal metastases in a multi-institutional cohort.

Patients and methods: From two academic centers, data of 155 patients with thoracic, lumbar or lumbosacral metastases was prospectively collected. In all patients, SINS was calculated by a spine surgeon, specialized in spine oncology and blinded for treatment outcome. Images from radiotherapy planning computed tomography (CT) scans were used. The highest SINS was recorded in case more than one lesion was irradiated. Patients who died within 4 weeks after radiotherapy $(\mathrm{n}=13,8 \%)$ or had an otherwise unknown pain response $(\mathrm{n}=18,12 \%)$ were excluded. In the 
remaining 124 patients, pain response was categorized, according to international guidelines, as complete, partial, indeterminate, or progression of pain. The association between SINS and pain response was estimated by multivariable logistic regression analysis, correcting for predetermined clinical variables.

Results: In total, 16 patients $(13 \%)$ experienced a complete and 65 $(52 \%)$ patients experienced a partial response. Of the patients who died within 4 weeks after radiotherapy, 6 patients had SINS of 7 or higher. Except for Karnofsky performance score, no significant differences in patients characteristics were found between responders and non-responders. SINS was associated with complete pain response [adjusted odds-radio (ORadj) 0.78; 95\% confidence interval (CI) 0.62-0.98], but not with overall pain response (ORadj 0.94; 95\% CI 0.81-1.10). For complete response, SINS improved the area under the ROC curve in addition to other clinical variables from 0.68 $(0.53-0.82)$ to $0.78(0.66-0.90)$ (Fig. a). SINS improved the prediction of response in addition to other clinical variables only marginally for overall response: the area under the receiver operating curve improved from $0.68(0.60-0.79)$ to $0.70(0.60-0.80)$ (Fig. b).

Conclusion: A lower SINS, indicating less spinal instability, is associated with a complete pain response after radiotherapy. This supports the hypothesis that pain resulting from mechanical spinal instability responds less well to radiotherapy compared to pain from local tumor activity. No association was determined between SINS and an overall pain response, which might indicate that this referral tool is not yet optimal for prediction of treatment outcome.

Disclosures: Author 1: none; Author 2: grants/research support: AOSpine Start Up Grant, consultant: AOSpine International; Author 3: grants/research support: OREF_-paid to institution, consultant: Medtronic, Nuvasive, royalties: Medtronic; Author 4: grants/research support: DePuy-Synthes Author 5: none; Author 5: none.
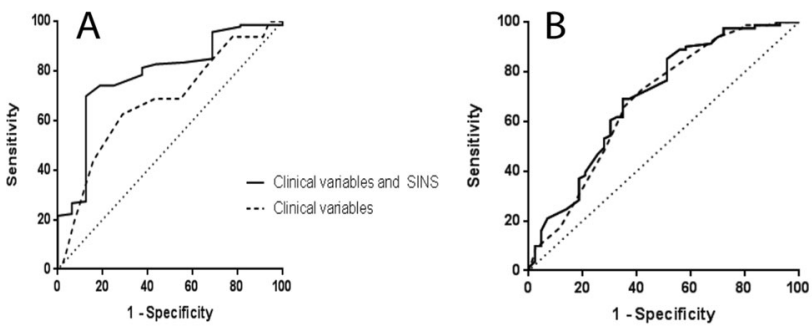

Figure ROC curves for the discriminative value of clinical variables (gender, primary tumor and performance status, dotted line) Figure ROC curves tor the discriminative value of clinical variables (gender, primary tumor and performance status, dotted line),
and SINS in addition to those clinical variables (solid line) in predicting complete pain response (A) and overall pain response (B:

\section{QF57}

\section{ABDOMINAL FAT DISTRIBUTION AS PROGNOSTIC FACTOR FOR SURVIVAL IN PATIENTS WITH SPINAL METASTASES}

Paul van Urk, Joanne van der Velden, Bart Pielkenrood, Jorrit-Jan Verlaan

Department of Orthopaedics, University Medical Center Utrecht, Utrecht, The Netherlands

Introduction: Predicting survival is important to optimize decision making for the treatment of patients with spinal metastases. Stabilizing spine surgery in these patients is generally considered to be inappropriate if anticipated survival is less than three months. Unfortunately, predicting survival is difficult for this patient category and an urgent need exists for more discriminative prognostic parameters. Abdominal fat distribution is the ratio between visceral fat area (VFA) and subcutaneous fat area (SFA). Recently, this parameter-as measured on diagnostic computed tomography (CT) scans-has shown to be a significant predictor of survival in patients with various primary cancers. However, the value of this parameter in patients with spinal metastases is unknown. If proven predictive of survival, selecting patients appropriately for surgery could be improved.

Objective: To evaluate if the VFA/SFA ratio is a prognostic factor for survival in patients with spinal metastases.

Methods: The design was an ongoing prospective cohort study. The VFA/SFA ratio was measured in patients with symptomatic spinal metastases who were referred to the departments of radiation oncology or orthopaedic surgery. The axial images at the L3 level from recent abdominal computed tomography scans, obtained for diagnostic or planning purposes, were used for the measurements. By setting the window level to enhance fat, the SFA and VFA could be delineated and its ratio calculated. Primary outcome was patient survival. Secondary study parameters included type of primary tumour, presence of visceral metastases and performance scores. Each parameter will be first examined in a univariate model, and then a multiple regression model will be set up by backward variable selection from the significant univariate analysis results.

Results: In total 343 patients with spinal metastases were included. The mean follow-up period was 10 months, with the range 1-72 months. The average age of participants was $65 \pm 13$ years and $63 \%$ of participants were male. Survival analysis showed an overall relative risk of death at 3 months of $1.2(95 \%$ CI $1.1-1.7, \mathrm{p}=0.04)$ in male participants and a relative risk of 1.8 (95\% CI 1.1-3.2, $\mathrm{p}=0.03$ ) in female participants. Multivariate data analysis identified VFA/SFA as an independent and significant factor in predicting survival. Also the karnofsky performance scale and primary cancer were independent significant factors.

Conclusion: Patients with spinal metastases and a higher visceral to subcutaneous fat area ratio have a significant worse survival rate.

Disclosures: Author 1: none; Author 2: none; Author 3: none; Author 4: none.

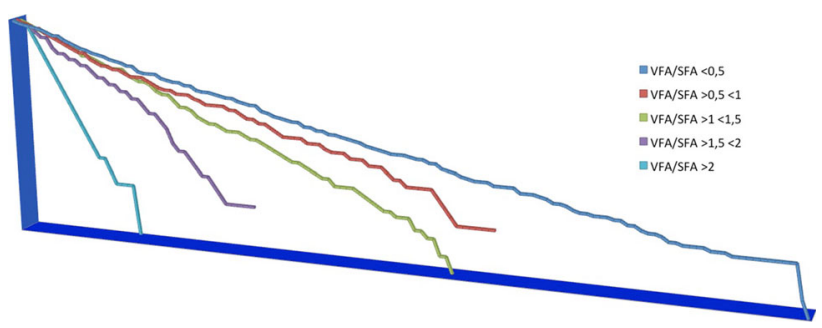

QF58

ANALYSIS OF FUNCTIONAL OUTCOMES AND COMPLICATIONS AFTER PALLIATIVE SURGERY AND RADICAL SURGERY IN METASTATIC SPINE DISEASE

Keun-Young Choi, Young-Hoon Kim, Kee-Yong Ha, Sang-Il Kim, Hyeong-Youl Park, Joo-Hyun Ahn

Department of Orthopaedic Surgery, Seoul University, Seoul, Korea

Introduction: Surgery for metastatic spinal disease (MSD) is challenging. Although the role of radiotherapy is increasing clinically, 
surgical treatment has still its own role in the treatment for MSD. However, there is a debate on surgical strategies. The purpose of this study to analyze postoperative functional outcomes according to the strategy of surgery.

Materials and methods: We retrospectively investigated 96 patients who underwent surgeries for their metastatic spinal tumors in a single institute between 2011 and 2015. Hematologic malignancies were excluded. Sixty patients belonged to palliative surgery group $(\mathrm{P})$ and 36 patients belonged to radical surgery group $(\mathrm{R})$. The postoperative changes of functional status and pain (visual analogue scale, VAS) were assessed. Primary endpoints were set as functional loss (neurologic deterioration) and reoperation for the index level. To identify the risk factors for primary endpoints, Cox hazards regression analysis was performed for multivariate analysis. Major complications within postoperative 30 days and survival rate were also analyzed. Survival analysis was performed using log rank test.

Results: The most common primary cancer was lung cancer $(36.5 \%)$ followed by hepatobiliary cancer $(27.1 \%)$. VAS decreased significantly in most patients $(83.3 \%)$ at postoperative 1-month. Eightyeight patients kept their ambulatory status after surgery, however, seven patients presented postoperative change (from non-ambulatory to ambulatory in four patients; from ambulatory to non-ambulatory in three patients). Both groups showed no significant difference of postoperative change about the ambulatory status within postoperative 30 days. Major complications developed in 24 patients (12 in $\mathrm{P}$ group, 12 in $\mathrm{R}$ group). Log rank test showed survival rates between two groups were not different significantly $(p=0.680)$. However, functional preservation rate at postoperative 12-month was 42.2 and $64.6 \%$ for $\mathrm{P}$ group and $\mathrm{R}$ group, respectively. Responsiveness to preoperative chemotherapy [hazard ratio $(\mathrm{HR}) 3.20, \mathrm{p}=0.013$ ] and the surgical strategy [hazard ratio (HR) $0.363, \mathrm{p}=0.027$ ] affected functional outcomes significantly.

Conclusion: Although major complications and ICU care were more frequently observed in group than $\mathrm{P}$ group, $\mathrm{R}$ group showed more excellent functional outcomes than $\mathrm{P}$ group. When surgical treatment is planned for MSD, especially in cases of unresponsiveness to preoperative chemotherapy, radical surgery following thorough preparation to reduce perioperative complications should be considered.

Disclosures: Author 2: none; Author 4: none; Author 5: none; Author 6: none.

\begin{tabular}{llll}
\hline & Hazard ratio & $95 \%$ CI & $P$ value \\
\hline $\begin{array}{l}\text { Surgical strategy } \\
\text { Palliative surgery }\end{array}$ & Reference & & \\
Radical surgery & 0.363 & $0.148-0.890$ & 0.027 \\
Responsiveness to preoperative CTx & & 0.013 \\
No CTx & Reference & & \\
Progression & 3.196 & $1.469-6.951$ & 0.003 \\
PR or SD & 1.865 & $0.598-5.819$ & 0.283 \\
\hline
\end{tabular}

\section{QF59 \\ RELATIONSHIP ANALYSES BETWEEN EPIDURAL SPINAL CORD COMPRESSION (ESCC) SCALE AND SEVERITY OF PARALYSIS OR IMPENDING PARAPLEGIA IN METASTATIC SPINE TUMOR}

Yasuaki Tokuhashi, Hiroshi Uei, Masafumi Maseda, Masashi Oshima

Department of Orthopaedic Surgery, Nihon University, Tokyo, Japan

Background/introduction: The severity of paralyses does not necessarily correlate with degrees of spinal cord compression by metastatic spine tumor. Also, the severity of paralyses varies by the affected level of the spine. Paralysis often may suddenly progress to paraplegia. However, there is few research that investigated the relationship among degrees of spinal cord compression by metastatic spine tumor, severity of paralysis, and impending paraplegia according to the affected level.

Purpose of the study: To evaluate the relationship between Epidural Spinal Cord Compression (ESCC) Scale (Bilsky MH, et al. J Neurosurg Spine, 2010) and severity of paralysis or impending paraplegia in metastatic spine tumor.

Materials and methods: A retrospective, single-institute, and radiographical study. We investigated 467 patients with spinal cord compression by metastatic spine tumor, whose degrees of spinal cord compression was higher than $1 \mathrm{~b}$ grade of Epidural Spinal Cord Compression (ESCC) Scale in the axial view of the T2 weighted MRI. The items of investigation were severity of paralyses, the level, direction of spinal cord compression, and ESCC Scale. The severity of paralyses was judged at the MRI imaging using the ASIA categorization. The symptomatic levels of paralyses were judged from maximal spinal cord compression in the ESCC Scale and neurologic findings. ESCC Scales were judged by three spine surgeons and done by the same decision with an ESCC Scale value more than two of them. Furthermore, they were judged it again 4 weeks later.

Also, the cases that worsened than in more than one grade of ASIA categorization and higher than grade $\mathrm{C}$ within 3 weeks after MRI imaging were considered.

Results: In the evaluation of the ESCC Scale, three examiners had not done a three-different decision, respectively, interobserver agreement was with a mean kappa of 0.90 and intraobserver was with a mean kappa of 0.95 . The symptomatic levels were 12 patients in the $\mathrm{C} 1-\mathrm{C} 2$, 65 patients in the C3-C6, 41 patients in the C7-T2, 149 patients in the T3-T10 (Table 1), 80 patients in the T11-L1 80, 113 patients in the L1-L5, and 9 patients in the S1. The borderline of occurred paralyses in more than $50 \%$ of patients matched between ESCC $1 \mathrm{~b}$ and $1 \mathrm{c}$ in the level C1-T2. In the level T3-L5, it matched the ESCC 1c. However, in the level L2-L5, the patients with even ESCC 3 did not have paralysis at all was found. There was no paralysis that was higher than ASIA C in the level S1. In the level C7-L1, there were high rates of higher than ASIA C paralyses in ESCC Scale 2 or 3. Also, the case that worsened than ASIA C with higher than 1 grade within 3 weeks after MRI imaging was 19 patients. In the group combined with the level of C7-T2, T11-L1 and T3-T10, the axial lesion of lateral or 
circumferential type and ESCC scale 2 or 3, higher than ASIA C paralyses occurred in more than $30 \%$ of patients of each group.

Conclusion: The state of the paralysis varied by the level, and that in neither level correlated with ESCC Scale. The paralysis development border, which occurred in more than $50 \%$ of patients, matched between ESCC $1 \mathrm{~b}$ and $1 \mathrm{c}$ in the level C1-T2, and did ESCC 1c in the level T3-L5. The high-risk criteria of rapid progressive paraplegia were combination with, C7-T2 or T11-L1 or T3-T10 in the level, lateral or circumferential type in the axial lesion, and ESCC scale 2 or 3.

Disclosures: Author 1: none; Author 2: none; Author 3: none; Author 4: none.

\section{Level T3-T10}

\begin{tabular}{|c|c|c|c|c|c|}
\hline ESCC & ASIA & A & $A+P$ & $A+L$ & $A+P+L$ \\
\hline \multirow[t]{2}{*}{$1 b$} & $E$ & 17 & 6 & 5 & 2 \\
\hline & D & & & 1 & \\
\hline \multirow{3}{*}{$(n=32)$} & c & & & 1 & \\
\hline & B & & & & \\
\hline & $A$ & & & & \\
\hline \multirow[t]{2}{*}{$1 \mathrm{c}$} & $E$ & 3 & & 6 & 2 \\
\hline & D & 2 & 1 & 1 & 1 \\
\hline \multirow[t]{3}{*}{$(n=23)$} & c & 1 & 1 & & 5 \\
\hline & B & & & & \\
\hline & A & & & & \\
\hline \multirow[t]{2}{*}{2} & E & & & & 1 \\
\hline & D & & 1 & 5 & 2 \\
\hline \multirow[t]{3}{*}{$(n=48)$} & C & 1 & & 13 & 20 \\
\hline & B & & & 2 & 3 \\
\hline & A & & & & \\
\hline \multirow[t]{2}{*}{3} & E & & & & 1 \\
\hline & D & & & & 2 \\
\hline \multirow[t]{3}{*}{$(n=44)$} & c & & & 7 & 12 \\
\hline & B & & & 11 & 9 \\
\hline & A & & & & 2 \\
\hline
\end{tabular}

A: Anterior, $A+P$ : Anterior + posterior, $A+L$ : Anterior + lateral, $A+P+L:$ Anterior + posterior + lateral

\section{QF60}

\section{TAG ARRAY GENE CHIP FOR SIMULTANEOUSLY RAPID DIAGNOSIS OF FIRST- AND SECOND-LINE ANTI- TUBERCULOSIS DRUG RESISTANCE IN MYCOBACTERIUM TUBERCULOSIS-A FEASIBILITY STUDY AUTHORS}

Wenjie Wu, Peng Cheng, Jingtong Lyu, Zehua Zhang, Jianzhong Xu

Department of Orthopaedics, Southwest Hospital, Third Military Medical University, Chongqing, China

Objective: We developed a Tag Array chip for detecting first- and second-line anti tuberculosis drug resistance in spinal tuberculosis and compared the analytical performance of the gene chip to that of phenotypic drug susceptibility testing (DST).

Methods: From June 2010 to November 2016. 187 consecutive clinically and pathologically diagnosed patients with spinal tuberculosis from Southwest Hospital, Chongqing were enrolled into the study. Specimens collected during surgery or percutaneous biopsy of the spine with CT guide from the tuberculosis patients were subjected to Mycobacterium tuberculosis species identification and drugresistance detection by the Tag Array gene chip, and evaluate the sensitivity and specificity of chip.

Results: A total of 137 patients was diagnosed drug-resistant tuberculosis. The detection of rifampicin (RFP), isoniazide (INH), fluoroquinolones (FQS), streptomycin (SM) resistance genes was highly sensitive and specific: however, for detection of amikacin (AMK), capreomycin (CPM), Kanamycin (KM), specificity was higher, but sensitivity was lower. Sensitivity for the detection of a mutation in the eis promoter region could be improved. The detection sensitivity of the EMB resistance gene was low, therefore it is easy to miss a diagnosis of EMB drug resistance, but its specificity was high. Conclusion: Tag Array chip can achieve rapid, accurate and highthroughput detection of tuberculosis resistance in spinal tuberculosis, which has important clinical significance and feasibility.

Disclosures: Author 1: none; Author 2: none; Author 3: none; Author 4: none; Author 5: none.

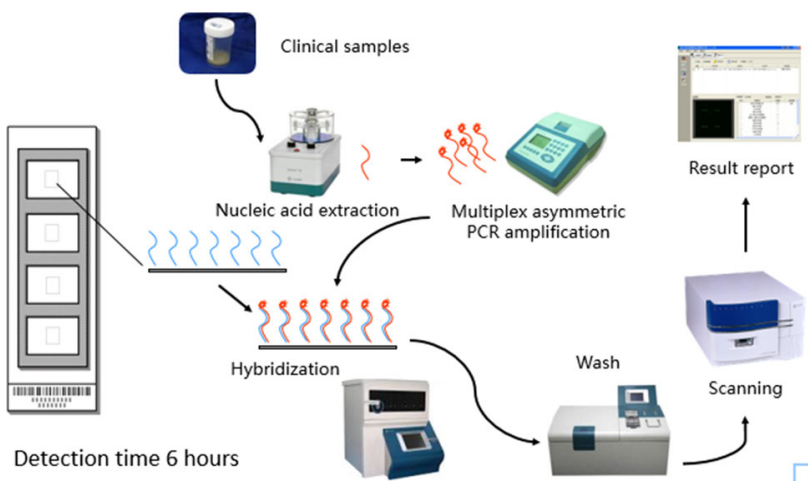

QF61

THE PREVALENCE OF DIFFUSE IDIOPATHIC SKELETAL HYPEROSTOSIS AMONG ACUTE TRAUMA PATIENTS IN SWEDEN

Atsuhiko Hirasawa, Yohan Robinson, Claes Olerud, Norimitsu Wakao, Mitsuhiro Kamiya, Mikinobu Takeuchi, Masataka Deie

Department of Orthopaedic Surgery, Aichi Medical University, Nagakute Aichi, Japan

Background: Diffuse idiopathic skeletal hyperostosis (DISH) can complicate non-surgical treatment of spinal fractures and often requires surgical intervention. Due to the underdiagnosis of DISH, treatment delays can occur. We previously reported a prevalence of DISH in Japan that was higher than that reported in other studies. As a comparison, we also performed a study in Sweden, which, similar to Japan, has an aging society.

Methods: Patients undergoing whole body computed tomography (CT) during trauma examinations at an acute outpatient clinic of Uppsala University Hospital in a 1-year period were eligible for inclusion. We excluded those $<40$ years old (Y/O), $\geq 90$ years/old, and those with previous spinal surgery. We investigated the prevalence of DISH by sex and age. DISH was determined by the presence of consecutive fused vertebral bodies per the criteria suggested by Resnick (Radiology 1976). 
Results: Eligible subjects (265 men and 153 women) ranged in age from 40 to 89 years, with a mean age of 63.4 years. Among men, 86 $(32.5 \%)$ were diagnosed with DISH, and the results by age $(40,50$, 60, 70, and 80 s) were: $6(10.7 \%), 13(22 \%), 35(46.1 \%), 17(34 \%)$, and $15(62.5 \%)$ patients, respectively. Among women, $16(10.5 \%)$ had DISH, distributed by the ages above as follows: $1(2.6 \%), 1$ (3.3\%), $2(6.7 \%), 6(22.2 \%)$ and $6(22.2 \%)$ patients, respectively. These results did not differ from those previously published from Japan (Fisher's exact test, men: $\mathrm{p}=1,0.27,0.12,0.06$, 1, women: $\mathrm{p}=0.49,0.62,0.5,0.8,0.3$, respectively).

Discussion: The prevalence of DISH has shown considerable differences based on ethnicity in some previous reports, describing a lower rate for Asians. However, we reported that the prevalence of DISH in Japan was higher than that in those reports, and this study showed a similar prevalence in Sweden. Therefore, we considered that ethnicity did not have a powerful influence on the prevalence of DISH. Some etiological factors have been reported, including age, sex, obesity, and metabolic abnormalities. This study showed that the prevalence of DISH increased with age and that there was a higher rate in men. However, there has been no detailed study into the relative influences of these factors. The World Health Organization has shown that rates of overweight and obesity are higher in Sweden than in Japan, so it may be that these factors did not strongly influence the etiology of DISH. It will likely be difficult to identify the etiology underlying DISH, as it is considered a multifactorial disease. However, some etiological studies have reported that abnormal glucose tolerance and abnormal lipid metabolism were associated with DISH, and these factors are considered to have strong etiological influences. Conclusions: We believe that our future research will provide important information not only for Japan but possibly worldwide as well, because this study showed that ethnicity was not a strong contributing factor.

Disclosures: Author 1: none; Author 2: other financial report: Medtronic, DePuy Spine; Author 3: other financial report: Depuyspine, Medtronic, AOSpine; Author 4: none; Author 5: none; Author 6: none; Author 7: none.

\section{QF62 \\ POSTERIOR REDUCTION AND STABILIZATION FOR THE TREATMENT OF COMPLETE THORACOLUMBAR FRACTURE-DISLOCATIONS}

Dingjun Hao, Xiaodong Wang, Hua Guo, Biao Wang

Honghui Hospital, Xi'an Jiaotong University College of Medicine, Xi'an, China

Purpose: The complete fracture-dislocations of thoracolumbar spine are considered as the most sever spine trauma, and usually result in neurological deterioration with spine deformity. The single center, large sample treatment study is rarely seen in the literature. We presented our experience of posterior reduction and stabilization in treatment of complete thoracolumbar fracture-dislocations.

Methods: 97 patients (69 males, 71.1\%) with complete fracturedislocations of the thoracolumbar spine were included from January 2008 to January 2013, and treated surgically by a posterior approach. Reduction and stabilization was achieved by means of pedicle screws and rods at the help of intraoperative traction. The operation time, intraoperative blood loss, AISA grade, Cobb angle, and complications were measured.

Results: With the use of posterior fixation and fusion, completely reduction was achieved in 89 patients $(91.8 \%)$, and partly reduction was achieved in 8 patients $(8.2 \%)$. Two complete paralyzed patients
( 1 female, 1 male) died of pulmonary embolism confirmed by the autopsy, which was due to the deep venous thrombosis, and had nothing to do with the surgical treatment. The average operation time was $165.6 \mathrm{~min}(98-328 \mathrm{~min})$, and the average intraoperative blood loss was $1226 \mathrm{ml}(130-2460 \mathrm{ml})$. The Cobb angle recovered postoperatively from the average of $29.5^{\circ}\left(8^{\circ}-53^{\circ}\right)$ to $2.9^{\circ}\left(-4^{\circ}\right.$ to $\left.10^{\circ}\right)$. At the latest follow-up, 28 patients $(29.5 \%)$ existed neurological functional recovery, and the remaining 67 patients $(70.5 \%)$ kept no significant changes of neurological function. No lossening, dislocation, breakage or other complications related to the internal implants were observed.

Conclusions: Posterior surgical approach is an efficient and safe way for the treatment of complete thoracolumbar fracture-dislocations. Successful reduction, satisfactory stability and neurological functional recovery can be obtained after surgery.

Key words: Thoracolumbar spine, Complete fracture-dislocations, Spinal surgery, Posterior approach.

Disclosures: Author 1: none; Author 2: none; Author 3: none; Author 4: none.

\section{QF63 \\ CLINICAL AND RADIOGRAPHIC OUTCOME OF TREATMENT OF THORACOLUMBAR FRACTURES WITHOUT NEUROLOGY. OPEN VS PERCUTANEOUS C-ARM: GUIDED VS PERCUTANEOUS 3D NAVIGATION: GUIDED PEDICLE SCREW FIXATION}

Marek Roclawski, Rafa Pankowski, Stanislaw Adamski, Wojciech Kloc, Witold Libionka

Department of Orthopaedics, Medical University, Gdansk, Poland; Department of Neurosurgery, Copernicus Hospital, Gdansk, Poland; Department of Neurology and Neurosurgery, Faculty of Medical Sciences, University of Warmia and Mazury, Olsztyn, Poland

Introduction: Recently, as an alternative for the open pedicle screw fixation (OPSF) of thoraco-lumbar fractures (TLF), percutaneous pedicle screw fixation (PPSF) has evolved to minimize soft tissue injury and perioperative morbidity. The purpose of this study was to prospectively compare the clinical and radiological outcomes of three different methods of neurologically intact TLF treatment: OPSF, minimally invasive two-plane conventional fluoroscopy-guided (FPPSF) and navigation-guided (N-PPSF).

Materials and methods: A total of 137 patients underwent either OPSF $(n=50)$ or F-PPSF $(n=39)$ or N-PPSF $(n=48)$ for stabilization of TLF between 2014 and 2016 in two Departments (Orthopaedics and Neurosurgery). Radiographs were obtained before surgery, immediately after surgery, and at the final follow-up for assessment of the restoration of the spinal column. For radiologic parameters, Cobb angle, vertebral wedge angle, and vertebral body compression ratio were evaluated on lateral thoracolumbar radiographs. As a method of clinical evaluation, the intraoperative blood loss, operation time, radiation dose, postoperative hospital stay, the postoperative scar size and perioprative complications.

Results: There were significant differences between open and both percutaneous methods of treatment in surgical blood loss and postoperative hospital stay in favour of percutaneous and without significant difference between F-PPSF and N-PPSF. The time of the surgery was the shortest in OPSF and F-PPSF. The radiation dose was both the highest for the patient and the lowest for the operative team in N-PPSF. The length of the postoperative scar was equal for all groups. There were five infections of the operative wound and one implant loosening in OPSF group without any significant 
complication. Postoperative VAS decreased significantly in all groups and there was a significant difference in favour of percutaneous techniques groups at the time of discharge and after 6 weeks and 3 and 6 months postoperatively. There were no statistical differences in VAS. For both F-PPSF and N-PPSF ODI was significantly better 6 weeks after the surgery than for OPSF without any difference between all groups at the final follow-up. The regional sagittal Cobb angle, vertebral wedge angle and anterior height of the fractured vertebra all significantly improved in each group immediately after the surgery and at the final follow-up.

Conclusion: Both percutaneous pedicle screw fixation techniques were comparable except the radiation dose for the operative team, which was the lowest in N-PPSF group. Although all three groups showed favorable outcomes at the final follow-up, F-PPSF and N-PPSF provided earlier pain relief, better functional improvement, less perioperative complications but worse early postoperative radiological outcome in comparison with OPSF.

Disclosures: Author 1: none; Author 2: none; Author 3: none; Author 4: none; Author 5: none.

\section{QF64 \\ RISK FACTORS FOR KYPHOTIC RECURRENCE AFTER TEMPORARY SHORT-SEGMENT FIXATION FOR THORACOLUMBAR BURST FRACTURES}

Hiroyuki Aono, Keisuke Ishii, Shota Takenaka, Yukitaka Nagamoto, Hidekazu Tobimastu, Masayuki Furuya, Tomoya Yamashita

Department of Orthopedic Surgery, Osaka National Hospital, Osaka, Japan

Introduction: Management for thoracolumbar burst fractures remains a matter of discussion. In annual meeting of Eurospine2015, we presented a comparative study of temporary short-segment fixation with or without vertebroplasty. In that presentation, the fractured vertebral body itself was reduced and maintained after surgery and the correction loss was observed at the disc level mainly after implant removal both with and without vertebroplasty. Therefore, we investigated the risk factors for kyphotic recurrence after temporary shortsegment fixation for thoracolumbar burst fracture.

Methods: This prospective multicenter study included 66 patients with thoracolumbar burst fracture who underwent surgery by ligamentotaxis procedure using Schanz screws. There are 44 men and 22 women with an average of 40 years. They were followed at least 1 year after implant removal. Their implants were removed around 1 year after initial surgery after confirming union of the fracture. We measured two angles in lateral radiograph in standing position (except before surgery), the vertebral body angle (VBA) which was measured between the superior and inferior endplates of the injured vertebra, and the kyphotic angle (KA) which was measured between the superior endplate of the intact vertebra cephalad to the fracture and the inferior endplate of the intact vertebra caudad to the fracture before and just after operation, approximately 1 year after initial operation and the final follow-up examination.

We also measured canal compromise ratio by CT scan.

A multiple logistic regression analysis was performed to identify the risk factors for postoperative correction loss. We defined $10^{\circ}$ or more correction loss in KA as kyphotic recurrence.

The factors that we evaluated were age, gender, affected level, combination of vertebroplasty, load-sharing score, AO classification (type A or B), preoperative VBA, KA, and canal compromise ratio. Results: VBA was corrected to $12.3^{\circ}$ after surgery and the total loss of correction was $0.8^{\circ}$
KA was corrected to $11.6^{\circ}$ after surgery and the total loss of correction was $9.1^{\circ}$. CT scans revealed a mean spinal canal narrowing of $48.3 \%$ before surgery, $26.2 \%$ after surgery, and $14.5 \%$ by the 2 -year follow-up examination, showing further improvement.

The multiple logistic regression analysis revealed preoperative KA $(P=0.48)$ and preoperative canal compromise ratio $(P=0.11)$ were risk factors for kyphotic recurrence. These results may suggest that the extent of disc destruction at the time of injury is closely related to the likelihood of kyphosis recurrence.

Conclusion: This study investigated the risk factors for kyphotic recurrence after temporary short-segment fixation for thoracolumbar burst fractures. Severe preoperative kyphotic angle and high canal compromise ratio by $\mathrm{CT}$ were the risk factors for postoperative kyphotic recurrence.

Disclosures: Author 1: none; Author 2: none; Author 3: none; Author 4: none; Author 5: none; Author 6: none; Author 7: none.

\section{QF65 \\ SINGLE STAGE POSTERIOR VERTEBRAL COLUMN RESECTION (PVCR) AND INTERNAL FIXATION FOR SURGICAL TREATMENT OF UNSTABLE THORACAL/ THORACOLUMBAR BURST FRACTURE}

Meric Enercan, Cem Sever, Selhan Karadereler, Sinan Kahraman, Onur Levent Ulusoy, Isik Karalok, Yesim Erol, Tunay Sanli, Azmi Hamzaoglu

Istanbul Spine Center, Istanbul, Turkey

Background: Single stage PVCR and internal fixation provides direct decompression of the neural structures, improves neurological deficit, corrects angular deformity, restores sagittal alignment, maintains immediate stability and anterior column support.

Purpose of the study: The ideal surgical treatment of unstable T/TL burst fractures with/without neurological deficit still remains debatable. The goals of surgical treatment are to decompress neural structures in case of neurologic deficit, restore kyphotic deformity and maintain stabilization of the vertebral column. Traditionally, these fractures were treated with combined anterior/posterior surgery. The aim of this study is to evaluate the clinical, neurological and radiological results of PVCR and posterior instrumentation in surgical treatment of unstable T/TL burst fractures.

Materials and methods: $44(27 \mathrm{~F}, 17 \mathrm{M})$ pts treated with PVCR and posterior instrumentation were reviewed. The surgical technique included segmental pedicle screw fixation, decompression of neural structures with wide laminectomy and PVCR at the fractured level, followed by placement of a titanium mesh or expandable cage for anterior column support. Preop, postop and latest f/up X-rays were evaluated for local kyphosis angle (LKA) and sagittal parameters. Neurological status were assessed according to ASIA Impairment Scale and functional status were assessed by Oswestry score.

Results: Ave age was 45.4 (22-83) years, ave f/up was 68.8 (26-158) months. Fractured level was thoracal in 14 and thoracolumbar in 30 pts. According to AOSpine TL Spine Injury Classification morphologic types; there were three Type A3, 17 Type A4, 4 Type B1, 6 Type B2, 5 Type B3, 9 Type C injuries. Ave. AOSpine TL Spine Injury score was 8.2 (5-13). Resections were one level in 41 pts and two-levels in 3 pts. Titanium mesh was used in 23 and expandable cage was used in 21 pts. for anterior column support. Ave LKA improved from $29.21^{\circ}$ to $4.39^{\circ} .13$ (\%62) of the 21 pts who had initial neurologic deficit (9 ASIA A, 2 ASIA B, 4 ASIA C, 6 ASIA D) showed at least one grade (ave. 1.76 grade) improvement on the final 
ASIA scale. Solid fusion was achieved in all pts without significant loss of correction at final f/up.

Conclusion: Single stage PVCR with posterior instrumentation is technically challenging in surgical treatment of unstable T/TL burst fractures. PVCR provides complete spinal canal decompression, achieve ideal kyphosis correction, restore anterior vertebral column and improves neurological deficit at least one grade (ave 1.76 grade). Disclosures: Author 1: none; Author 2: none; Author 4: none; Author 5: none; Author 6: none; Author 7: none; Author 8: none; Author 9: consultant: MEDTRONIC

\section{QF66 \\ IS THE PELVIC INCIDENCE RELATED WITH THE RESORPTION OF HERNIATED LUMBAR DISC?}

Nam-Su Chung, Chang-Hoon Jeon, Han-Dong Lee, Young-Uk Seo

Department of Orthopaedic, Ajou University Medical Center, Suwon, South Korea

Purpose: The study was designed to evaluate the relationship between the pelvic incidence and the resorption of herniated lumbar disc.

Introduction: The resorption of the herniated disc has been considered as an important factor to predict the favorable outcome. Age, the relief of symptoms, the types of disc herniation, Modic change and the rim enhancement around herniated disc in gadolinium-enhanced magnetic resonance image (MRI) have been found to be related with disc resorption. Pelvic incidence, which is considered as a constant radiographic factor, has been found to be related with the disc degeneration and the level of disc herniation. However, there is no literature showing the relationship between pelvic incidence and the resorption of herniated lumbar disc.

Methods: In this study, there were 56 patients who had lumbar disc herniation, which was diagnosed with the clinical symptoms, neurologic examination and with MRI. All patients were re-evaluated with MRI at the 1-year follow-up. According to the pelvic incidence (PI), we classified the subjects into high PI group (PI $>45^{\circ}$ ) and low PI group $\left(\mathrm{PI} \leq 45^{\circ}\right)$. Spino-pelvic parameters and lumbar lordosis were measured on the initial radiograph. Affected level, the types and volume of disc herniation, the grade of disc degeneration (Pfirrmann's grade), the types of Modic change, and the rupture of posterior longitudinal ligament (PLL) were measured on the initial MRI. Two groups were compared using a t-test and qui square test depending on the type of variables.

Results: There were no significant differences regarding age, sex, affected level, the types of disc herniation, the grade of disc degeneration, the types of Modic change and the rupture of posterior longitudinal ligament between two groups (All $\mathrm{P}$ values $>0.05$ ). All results regarding the change of disc volume and pelvic parameters are shown in Table 1 .

Conclusions: In low pelvic incidence group, the initial herniated disc volume was larger and the resorption volume of herniated disc was larger. Low pelvic incidence may be related with the occurrence of disc herniation with larger volume and the herniated disc resorption. Pelvic incidence can be helpful to predict the prognosis of herniated disc.

Disclosures: Author 1: none; Author 2: none; Author 3: none; Author 4: none.
Table 1. pelvic parameters and the change of disc volume between high PI group and Low PI group.

\begin{tabular}{|c|c|c|c|}
\hline & High PI (n=30) & Low PI $(n=26)$ & $P$ value \\
\hline \begin{tabular}{|l|} 
Pelvic incidence (") \\
\end{tabular} & $51.51 \pm 7.29$ & $34.86 \pm 7.46$ & $0.001^{\circ}$ \\
\hline \begin{tabular}{|l|} 
Pelvic tilt (") \\
\end{tabular} & $17.14 \pm 7.69$ & $9.58 \pm 7.14$ & $0.012^{*}$ \\
\hline \begin{tabular}{|l|} 
Lumbar lordosis (") \\
\end{tabular} & $47.62 \pm 11.20$ & $31.22 \pm 15.63$ & $0.003^{\circ}$ \\
\hline $\begin{array}{l}\text { Initial herniated } \\
\text { disc volume }\left(\mathrm{mm}^{3}\right)\end{array}$ & $637.53 \pm 237.37$ & $1065.66 \pm 616.99$ & $0.020^{\circ}$ \\
\hline $\begin{array}{l}\text { Last herniated } \\
\text { disc volume }\left(\mathrm{mm}^{3}\right)\end{array}$ & $591.29 \pm 301.35$ & $580.14 \pm 419.85$ & 0.936 \\
\hline $\begin{array}{l}\text { Resorption volume of } \\
\text { herniated disc } \\
\left(\mathrm{mm}^{3}\right)\end{array}$ & $46.24 \pm 300.39$ & $485.52 \pm 423.19$ & $0.004^{\circ}$ \\
\hline $\begin{array}{l}\text { Herniated disc } \\
\text { resorption rate (\%) }\end{array}$ & $1.91 \pm 44.06$ & $41.57 \pm 27.89$ & $0.010^{\circ}$ \\
\hline
\end{tabular}

*PI, pelvic incidence.

*Unless otherwise noted, data are means \pm standard peviations

\section{QF67}

AN ANATOMIC EVALUATION OF RETROPERITONEAL ORGANS FOR LATERAL APPROACH SURGERY: PROSPECTIVE IMAGING STUDY USING COMPUTED TOMOGRAPHY IN LATERAL DECUBITUS POSITION

Jun Ouchida, Tokumi Kanemura, Kotaro Satake, Hiroaki Nakashima, Naoki Segi

Department of Orthipedic Surgery, Konan University, Nagoya, Japan

Introduction: Lateral interbody fusion (LIF) is an established surgical procedure that is minimally invasive and highly effective for indirect decompression with lumbar spinal stenosis. However, use of the retroperitoneal approach has the potential to result in critical intraoperative complications like colonic injury, ureter injury, injury to great vessels or injury to other retroperitoneal organs. Though the surgical procedure is performed in the lateral decubitus position, little mention has been made of anatomical measurements of retroperitoneal organs in this position. The purpose of this study is to investigate the distribution of retroperitoneal organs while using the retroperitoneal approach in the lateral decubitus position.

Materials/methods: A total of 81 consecutive patients scheduled to undergo lateral approach surgery including LIF and lateral corpectomy were enrolled. We performed computed tomography with lateral decubitus positioning (L-CT) to detect vascular malformations and assessed the position of retroperitoneal organs while using the retroperitoneal approach. The survey included the kidney (posterior edge), the descending colon (posterior edge), the ureter, and the gonadal artery. The anteroposterior position of the organs were divided into four zones with regard to the vertebral body: A was anterior to the anterior edge of the vertebral body, AL was from the anterior edge to the middle line of the vertebral body, PL was from the middle line to the posterior edge of the vertebral body, and $\mathrm{P}$ was posterior to the posterior edge of the vertebral body. The positions of the descending colon, the ureter, and the gonadal artery were evaluated at each disk level (L1/2-L4/5). These measurements obtained using L-CT were compared with those obtained in the conventional supine position (S-CT).

Results: The deviation in S-CT and L-CT (A:AL:PL:L, \%) was $0: 1: 6: 93$ and $1: 20: 36: 43$ for the kidney, 20:27:31:21 and 64:17:11:3 for the descending colon, 36:57:6:1 and 84:16:0:0 for the ureter, and 
70:27:2:1 and 97:3:0:0 for the gonadal artery. Forty-one (67\%) of the 61 disk levels recorded for the descending colon in zone P under $\mathrm{S}-\mathrm{CT}$ still remain posterior to the anterior edge of the vertebral body (zones AL, PL, or P) even under L-CT.

Conclusions: We found anterior migration in L-CT in all retroperitoneal organs. LIF in the lateral decubitus position reduces the risk of injuries to the retroperitoneal organs to some extent. However, a certain percentage of the kidney and the descending colon still remain in the way while performing LIF. We discussed the importance of preoperative imaging evaluation and meticulous attention during the surgery in cases where the descending colon extends outside zone $\mathrm{P}$ under S-CT.

Disclosures: Author 1: none; Author 2: consultant: NuVasive, Medtronic; Author 3: none; Author 4: none; Author 5: none.

\section{CERVICAL SPINE}

\section{QF68 \\ CHARACTERISTICS OF CERVICAL SPINE INJURY IN THE AGED SOCIETY (THE AGING RATE: $>33.3 \%$ )}

Toshiyuki Dokai ${ }^{1}$, Toru Yonei ${ }^{1}$, Tetsuya Otsuka ${ }^{1}$, Ryunosuke Kono ${ }^{1}$, Hideki Nagashima $^{2}$

${ }^{1}$ Department of Orthopedic Surgery, Masuda Red Cross Hosp, Masuda, Japan; ${ }^{2}$ Department of Orthopedic Surgery, School of Medicine, Tottori University, Yonago, Japan

Background: Japan is entering an aging society, and the health care zone that our hospital is in charge is becoming more aged; the aging rate is already $33.3 \%$ in 2010 . The population in our health care zone is approximately 65,000 and is gradually decreasing: however, the aging rate is gradually becoming higher. Medical self-sufficiency rate of our health care zone is $89.0 \%$. Therefore, to investigate cervical spine and injury (CSI) in our hospital is useful in predicting the characteristics of CSI in the aged society.

Methods: There were 57 cases of CSI between January 2009 and September 2016. We retrospectively analyzed age, gender, injury mechanism, injured level, Frankel classification (at injury, final follow-up), with or without bone injury, and comorbidities. We performed two studies.

(Study 1) We examined the incidence of CSI and discussed the characteristics in the aging society. We divided into the two groups: the former group (from January 2009 to December 2012) and the latter group (from January 2013 to September 2016), and compared the groups.

(Study 2) We divided the subjects into the two groups; Group A (ages were 80 years and over) and Group B (ages were under 80 years), and compared the two groups.

Results: (Study 1) There were 27 cases of CSI in the former group and 40 cases in the latter group. There was no statistically difference between the two groups.

(Study 2) There were 18 cases of CSI in the Group A (the average age: 87.1 years) and 49 cases in the Group B (the average age: 68.6 years). There were statistically significant differences in age $(p=0.036)$, Frankel A at injury ( 8 cases in the Group A and 3 in the Group B; $p=0.001)$, and the number of death within 6 months ( 5 cases in the Group A and none in the Group B; p = 0.025) between the Group A and $\mathrm{B}$. The recovery rate of paralysis (at least 1 grade or over with Frankel classification) was $16.7 \%$ (3 out of 18 cases) in the Group A and $51.0 \%$ ( 25 out of 49 cases) in the Group B. The recovery rate of the Group A was significantly lower than the Group B. There were 5 mortality cases in the Group A and none in the Group B. The details of death were pneumonia in 3 cases, respiratory insufficiency in 1 case and gastric ulcer in 1 case. All of the mortality cases were classified as Grade A classification of Frankel at injury

Conclusions: According to our results, the annual incidence of CSI is estimated at 185 cases per 1 million populations, which is higher than the previous reports. There was no statistically difference in the injury mechanism and the presence of bone injury in the group A and B: therefore, at the aged society, the active elderly people will receive CSI by high energy accidents, as same as young people. Furthermore, the elderly will have the tendency of severe paralysis, compared with the young people. In addition, it is a serious problem that mortality within 6 months is high in the cases with CSI in the elderly.

Disclosures: Author 1: none; Author 2: none; Author 3: none; Author 4: none; Author 5: none.

\section{QF69 \\ USE OF COMI FOR COMPARISON OF ANTERIOR CERVICAL DISCECTOMY AGAINST POSTERIOR FORAMINOTOMY AS TREATMENT FOR CERVICAL RADICULOPATHY}

Anastasios Benjamin Konteas, Nick Carleton-Bland, Maggie Lee, Simon Clark, Martin Wilby

The Walton Centre Liverpool NHS Trust, Liverpool, England

Background context: Cervical radicular compression is a commonly encounter condition in spinal surgical practice. Radiculopathy is debilitating with recognised affects on social functioning and quality of life.

Surgical treatment aims to decompress the root via either anterior cervical discectomy ("ACDF") or posterior cervical foramenotomy ("PCF"). The choice of which surgical strategy is controversial, as there is little conclusive evidenced opinion comparing efficacy or sequelae of these approaches using validated outcome measures.

Purpose: To compare PCF and ACDF with a validated outcome measure to allow comparison of the two techniques.

Study: Review of a single centre prospectively collected COMI data on ACDF and PCF performed between 2012 and 2014

Outcome measure: Self-reported Core Outcome Measurement Index ("COMI") at 0,3,12 and 24 month post procedure.

Methods: Eight surgeons were involved in the allocation of either anterior or posterior surgery as was the surgeon's preference. All candidates were contemporaneously COMI assessed. Data acquisition was at clinic appointment or via postal methods.

Results: Complete datasets were acquired in $49 \mathrm{PCF}(41 \mathrm{M} 22 \mathrm{~F})$ and 262 ACDF (M F).

For ACDF COMI-mean scores: 7.6 (0 months)-5.1(3 months) -4.2 (12 months).

For PCF COMI-mean scores: 7.2(0 months $)-5.6(3$ months $)-$ 4.7(12 months).

Significant complication rate was $3 \%$. Patient reported outcome measures are also discussed.

Conclusion: COMI scores suggest similar efficacy of the two procedures; both procedure produce a clinical and statistically significant improvement.

Notably patients who have undergone PCF reach their maximal improvement at 3 months. This is in contrast to ACDF patients, who continue to improve up to 12 months post procedure.

Interpretation is that sub-optimally treated radiculopathy in the ACDF group can be treated conservatively as there is statistically significant improvement up to a year post procedure. 
Of interest the patient reported outcome measures ("PROMS") gave a complication rate of $30 \%$ as compared to a clinical complication rate of $3 \%$, indicating need for improvement in patient consent processes.

Strengths of this study are the use of a validated assessment tool with large groups and ample follow up period. Limitations include incomplete COMI data, lack of blinding and group size disparity.

Disclosures: Author 1: none; Author 2: none; Author 3: none; Author 4: grants/research support: medtronic, consultant: medtronic; Author 5: none.

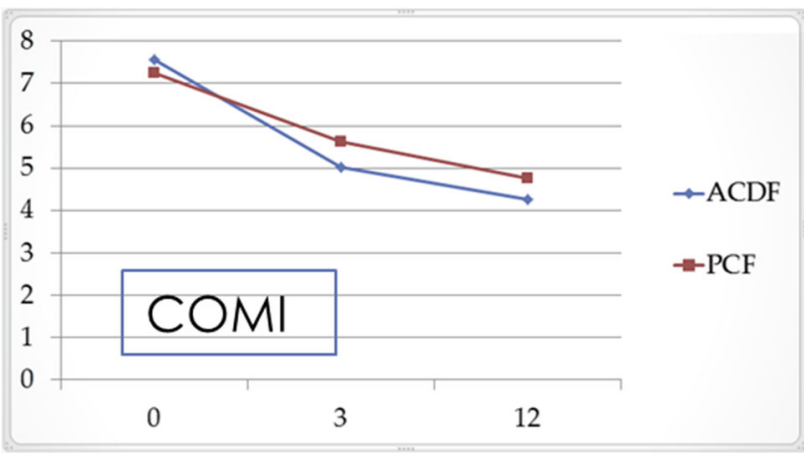

\section{QF70 \\ MORTALITY AND UNEXPECTED SURGERY RATE IN PATIENTS WITH CERVICAL MYELOPATHY SURGICALLY TREATED. REVIEW OF 305 CASES OF AN INSTITUTION}

Manuel Ramírez Valencia, Xavier Plano Jerez, Antonia Matamalas Adrover, Ana García de Frutos, Pilar González-Tartière, Joan Bagó Granell, Ferran Pellisé Urquiza, José Manuel Casamitjana Ferrándiz

Vall d'Hebron University Hospital, Barcelona, Spain; Teknon Clinic, Barcelona, Spain

Introduction: Unexpected Surgery Rate (unplanned surgery) is increasingly used as a quality measure of a surgical service. Their rate of occurrence and impact on mortality in cervical spine surgery is not clearly defined.

Purpose of the study: To review and calculate mortality and unplanned surgery (US) rate in patients operated for cervical myelopathy.

Materials and methods: US was defined as any surgical procedure required to treat a direct or indirect complication after "index" surgery in the first 30 days. Retrospective cohort study using the database of patients operated for cervical myelopathy between January 2005 and March 2014, excluding those treated surgically through double approach. Comorbidities, ASA, obesity, smoking, type of surgery and medical or surgical complications were collected. Mortality in the first 90 days and US rate were calculated. Satisfaction scale and final outcome were measured by GAT (Global Assessment Tool). Student t, Mann-Whitney U, Chi squared or F tests were used. We used bootstrap techniques to achieve $95 \%$ confident interval.

Results: 305 patients. Average age 57.7 years (range 27-86), 66\% men. $42 \%$ smokers. $88 \%$ ASA 2 or $3.78 \%$ had some comorbidity. $29 \%$ of patients presented medical and $8.9 \%$ surgical complications. Anterior approach was used in $65.9 \%$.

The mortality rate was $1.1 \%$ (95\% CI 0-2.4) and age was the only risk factor, with an age difference of 18.3 years (95\% CI 13.9-22.9) between the mortality vs no-mortality group, being significantly older in the mortality group.
US rate was $2.6 \%$ (95\% CI 1-4.6). The most common cause was postoperative infection, followed by retropharyngeal haematomas. The parameters that showed relationship were DM (OR 2.2; $95 \% \mathrm{CI}$ 0.9-5.5) and surgical complications (OR 6.5; 95\% CI 1.0-40).

Neither surgical approach nor implant complications were associated with US. Presence of US did not influence the final outcome. Conclusion: Mortality rate in our sample was $1 \%$ and it was mainly associated with patients over 70 years. The risk of requiring US was between 1 and $4.6 \%$, although US did not seem to have impact in the final outcome or patient satisfaction.

Disclosures: Author 1: consultant: MBA; Author 2: none; Author 3: none; Author 4: grants/research support: De Puy, consultant: Stryker; Author 5: none; Author 6: none; Author 7: grants/research support: DepuySpine Synthes, Medtronic, consultant: DepuySpine Synthes, Zimmer-Biomet, Kisko; Author 8: none.

\section{QF71 \\ RELATIONSHIP BETWEEN CLINICAL AND RADIOLOGICAL RESULTS IN PATIENTS SUBMITTED TO ANTERIOR CERVICAL DISCECTOMY AND FUSION}

Bruno Maia, Pedro Carvalhais, Luis Teixeira

Spine Center, Cirurgia da Coluna, Coimbra, Portugal

Background: Degenerative cervical disease is a major cause of radiating upper extremity pain associated with neurological abnormalities. Anterior cervical discectomy and fusion (ACDF) has been accepted as an effective treatment on a various spinal cervical abnormality such as spondylosis, herniated discs, fractures, neoplastic lesion. After complete decompression of the involved disc space, diverse graft materials such as an autogenous bone block, allograft, or cages are inserted in intervertebral spaces. Those materials are not only for fusion but also for indirect decompression by distraction. However, oversized grafts can cause over distraction of facet joins leading to post-operative neck pain

Objectives: The purpose of this study was to compare the relationship between postoperative increases in intervertebral disc space height, interfacet distance (IFD) and lordosis with clinical results in patients undergoing anterior cervical discectomy and fusion (ACDF) for degenerative cervical disease.

Study design and methods: A total of 100 patients were included in this study. Radiologically, IVH and IFD and cervical lordosis (C1 to C7) were measured preoperatively and postoperatively. Clinical evaluation according to visual analogue scale (VAS) scores and neck disability index (NDI) scores preoperatively and postoperatively for 1 year were accessed. The relationship between radiological parameters, and clinical scores were analyzed

Results: In terms of Radiological parameters The mean increase for IVH was $31 \mathrm{~mm}$, for IFD was $4.6 \mathrm{~mm}$ and for cervical lordosis was $7^{\circ}$. The VAS and NDI scores had statistically significant improvements comparing pre-operative and follow-up values (VAS: $6.16 \pm 2.693$ vs $1.05 \pm 1.580, p=0.05 ;$ NDI: $43.65 \pm 17.439$ vs $15.26 \pm 10.994, p=0.01$ ); No significant relationship was observed between the radiological evaluation results regarding intervertebral height interfacet distance or lordosis and clinical changes in VAS or NDI scores. However we found a statistically significant difference between IVH and lordosis pre and post-surgery.

Conclusions: The increase in intervertebral space, interfacet distance and lordosis by the insertion of larger cages were not related with clinical improvements in VAS and NDI score during the follow-up period.

Disclosures: Author 1: none; Author 2: none; Author 3: none. 


\section{QF72}

\section{LONG-TERM OUTCOMES OF ARTHROPLASTY FOR CERVICAL MYELOPATHY VERSUS RADICULOPATHY, AND ARTHROPLASTY VERSUS ARTHRODESIS FOR CERVICAL MYELOPATHY}

Matthew F. Gornet, K. Daniel Riew, Todd H. Lanman, J. Kenneth Burkus, Scott D. Hodges, Jeffrey R McConnell, Randall F. Dryer, Anne G. Copay, Francine W. Schranck

\section{The Orthopedic Center of St. Louis, St. Louis, USA}

Background context: While cervical disc arthroplasty (CDA) has been used for the treatment of cervical disc disease with radiculopathy or myelopathy, concerns remain about the appropriateness of CDA to treat patients with myelopathy.

Purpose: This study was to compare long-term safety and effectiveness of CDA in patients with myelopathy vs radiculopathy.

Materials and methods: Retrospective analysis of prospectively collected 84-month outcomes data from an FDA IDE clinical trial comparing CDA to ACDF for the treatment of cervical disc disease at 2 adjacent levels: NDI, neck and arm pain, SF-36 PCS, neurological status, adverse events, secondary surgeries at index and adjacent levels. A total of 397 patients were enrolled with a diagnosis of radiculopathy, myelopathy, or both: 287 radiculopathy alone, and 110 myelopathy alone or myelopathy with radiculopathy. Two comparisons were performed. First, CDA outcomes were compared between myelopathy (including myelopathy only and both myelopathy and radiculopathy) and radiculopathy patients. Second, the outcomes of $\mathrm{CDA}$ and $\mathrm{ACDF}$ were compared for myelopathy patients (including myelopathy only and both myelopathy and radiculopathy).

Results: There were no preoperative differences for the 1 st comparison and 2 days comparison for NDI, neck and arm pain, and PCS scores. All patient groups significantly improved for NDI, neck and arm pain, and PCS scores from preoperative to 84 months.

First comparison: The myelopathy and radiculopathy groups showed similar improvement for NDI (37.8 vs $35.8, \mathrm{p}=0.352$; myelopathy vs radiculopathy, respectively), neck pain (12.0 vs $12.1, \mathrm{p}=0.477)$, arm pain (11.6 vs 9.6, $\mathrm{p}=0.480)$, and PCS (14.1 vs $13.7, \mathrm{p}=0.863$ ). The two groups had similar proportions of patients who maintained or improved their neurological status $(87.2$ vs $93.5 \%, \mathrm{p}=0.218)$, similar rates of serious adverse events (AEs) $(54.5$ vs $57.5 \%, p=0.291)$ and similar rates of secondary surgeries at index (3.7 vs $4.4 \%$, $\mathrm{p}=0.839)$ and adjacent levels (3.7 vs 7.6\%, $\mathrm{p}=0.367$ ).

Second comparison: The CDA and ACDF groups showed similar improvement for NDI (37.8 vs 31.1, $\mathrm{p}=0.147$; CDA vs ACDF, respectively), neck pain (12.0 vs $10.4, \mathrm{p}=0.337)$, and arm pain (11.6 vs $11.4, \mathrm{p}=0.791)$, PCS (14.1 vs $11.2, \mathrm{p}=0.363)$. The two groups had similar proportions of patients who maintained or improved their neurological status $(87.2$ vs $96.2 \%, p=0.409)$ and similar overall rates of secondary surgeries at the index levels (3.7 vs $9.4 \%$, $\mathrm{p}=0.374$ ), and similar rates of secondary surgeries at adjacent levels ( 3.7 vs $15.4 \%, \mathrm{p}=0.088$ ). Compared to $\mathrm{ACDF}$, the CDA group demonstrated lower rates of serious AEs $(54.5$ vs $65.9 \%, p=0.019)$. Conclusions: Long-term results show that CDA is safe and effective for the treatment of myelopathy. Myelopathy patients gain similar improvement from CDA to patients with radiculopathy only. Furthermore, myelopathy patients report similar levels of improvement from CDA compared with ACDF, but suffer fewer serious AEs

Disclosures: Author 1: consultant: Medtronic, K2M, stock/shareholder: Bonovo, International Spine and Orthopedic Institute, LLC, Nocimed, OuroBoros, Viscogliosi Bros Venture Partners LLC, royalties: Medtronic; Author 2: grants/research support: Lahey Clinic/ PCORI, consultant: Biomet, Medtronic, stock/shareholder: Osprey, Expanding Orthopedics, Spineology, Spiral Kenetics, Nexgen Spine,
Amedica, Vertiflex, Benvenue Paradigm Spine, PSD, royalties: Biomet, Medtronic, other financial report: Zeiss (Taught Webinar); Author 3: consultant: Nuvasive, Medtronic, Choice Spine, royalties: Stryker, Medtronic, Nuvasive; Author 4: grants/research support: Medtronic, consultant: Medtronic; Author 5: grants/research support: Medtronic, consultant: Medtronic; Author 6: consultant: Globus Medical Inc., stock/shareholder: Globus Medical Inc., royalties: Globus Medical Inc.; Author 7: consultant: Innovasis, royalties: Globus, Nuvasive; Author 8: none; Author 9: grants/research support: Predicted, Reported, and Observed Outcomes Foundation, stock/ shareholder: Nocimed, other financial report: Medtronic (vendor); Zimmer/Biomet (vendor); Nocimed (vendor); Aesculap (vendor).

\section{QF73 \\ COMPARING COST-EFFECTIVENESS OF ARTHROPLASTY WITH FUSION IN TREATMENT FOR ONE LEVEL CERVICAL RADICULOPATHY}

Oddrun A. Fredriksli, Jarle Sundseth, Frode Kolstad, Hege Andresen, Kay Müller, Erling Myrseth, John A. Zwart, Oystein P. Nygaard, Lars G. Johnsen

Department of Neurosurgery/NTNU, Norwegian University of Science and Technology, Trondheim, Norway

A multicentre randomized controlled trial.

Background/introduction: Operative treatment for cervical radiculopathy is rapidly increasing. Fusion is the most common method, however in recent years, use of cervical arthroplasty has increased worldwide.

Purpose of the study: The objective of this study was to compare the cost-effectiveness of cervical arthroplasty with fusion in patients with symptomatic, single level C6 or C7 radiculopathy.

Materials and methods: Patients were randomly allocated to arthroplasty $(n=68)$ or fusion $(n=68)$. Randomization was blinded for patients. Surgical team was blinded for type of implant until after decompression. Assessment of

EuroQol-5D-3L and Short form-6D at 3, 6, 12 and 24 months, quality-adjusted life years (QALYs) after 2 years. Relevant direct and indirect costs were assessed from a societal perspective. Cost-effectiveness was presented by the incremental cost-effectiveness ratio (ICER).

Results: The dropout rate was $11.8 \%$ in the arthroplasty group and $10.3 \%$ in the fusion group.

Mean QALYs gained (SD) for EQ-5D: $1.41(0.36)$ in the arthroplasty group and $1.35(0.37)$ in the fusion group, a difference of $0.05(95 \%$ CI 0.08, 0.18). QALYs gained (SD) using SF-6D: $1.41(0.21)$ in the arthroplasty group and $1.40(0.19)$ in the fusion group, a difference of 0.003 (95\% CI $0.068,0.074)$.

Mean total cost per patient in the arthroplasty group was $€ 45,760$ $(30,906)$ compared with $€ 39,137(29,750)$ in the fusion group. Differences between the groups for total costs per patient were $€ 6623$.

$(95 \% \mathrm{CI}-4300,17,545), \mathrm{p}=0.23$. Incremental cost-effectiveness ratio (ICER) for arthroplasty was $€ 126,293 /$ QALY when using EQ-5D-3L (arthroplasty not cost-effective).

Conclusions: Patients in both groups have a large improvement in quality of life. However, there is no difference in quality of life in the two groups after 2 years.

The higher cost of arthroplasty is mainly due to implant cost.

This study's strength, was the comprehensive collections of healthcare utilization data within a randomized controlled study design. 
In this study, arthroplasty was not cost-effective compared with fusion after 2 years when using EQ-5D-3L for assessing QALYs gained and a willingness to pay of $€ 66,900 / \mathrm{Q} A L Y$.

Disclosures: Author 1: none; Author 2: none; Author 3: none; Author 4: none; Author 5: none; Author 6: none; Author 7: none; Author 8: none; Author 9: none.

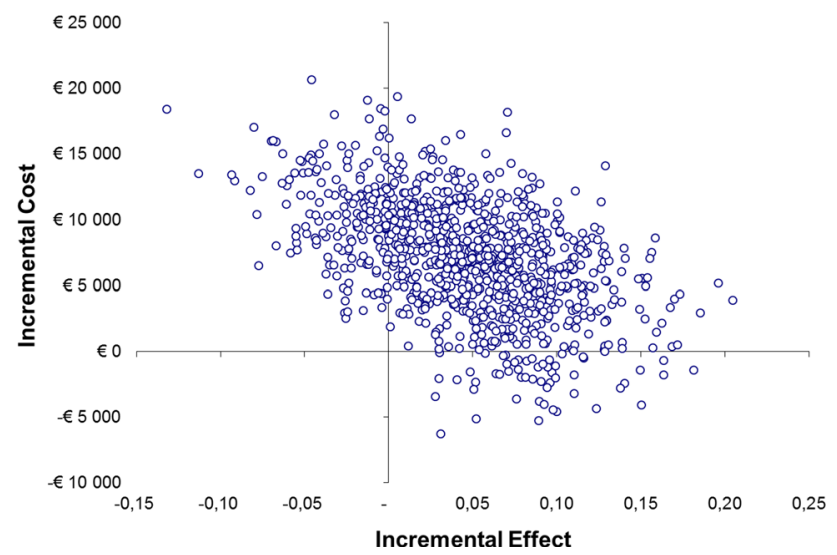

QF74

\section{ASSESSING FUSION, SUBSIDENCE AND ALIGNMENT IN THE CERVICAL SPINE: COMPARISON OF DIFFERENT MEASUREMENTS ON PLANS RADIOGRAPHS}

Ehab Shiban, Jens Lehmberg, Bernhard Meyer

Department of Neurosurgery, Technical University of Munich, Munich, Germany

Introduction: There are different definitions of arthrodesis, alignment and subsidence in the cervical spine. We aimed to compare the accuracy of different objective radiographic techniques in after anterior cervical discectomy and fusion and correlated those with clinical outcome.

Methods: A retrospective analysis of 200 radiographs of patients following ACDF with stand alone-PEEK cages with a minimum follow up of 12 months was performed. For assessment of fusion one measurement was obtained from plain radiographs: the presence or absence of bridging bone in the operated segment. Three measurements were obtained from flexion-extension radiographs: Cobb angle and the distance between the tips as well as the distance between the bases of the spinous processes of the operated segment. Measurement of more than $2 \mathrm{~mm}$ between the bases of the spinous processes was defined as pseudarthrosis as recommended by the joint guidelines committee of the AANS/CNS. Cervical alignment was assed using the Katsuura, Lang and Cobb methods. Subsidence was measured with the mochida method, ventral segmental height reduction and dorsal segmental height reduction. Correlation between the different radiological methods and clinical outcome was performed.

Results: Fusion rates varied greatly depending on the method used (from 43.9 to $89.4 \%$ ). None of the methods used had any correlation with clinical outcome. The rate of improvement or stability of the cervical alignment was seen in $83.2,36.6$ and $43.3 \%$ using the Laing, Katsuura and Cobb methods, respectively. Neck pain correlated with the cervical alignment using the Cobb $(\mathrm{p}=0.27)$ and the Katsuura $(p=0.034)$ assessment methods but failed to correlate with Laing assessment method $(p=0.102)$. Subsidence rates were 62,48 and $27 \%$ using the mochida, ventral and dorsal segmental height reduction assessment methods, respectively. Subsidence using the mochida method correlated with neck pain at last follow up $(\mathrm{p}=0.047)$, but did not correlate with clinical outcome with the other measurement methods.

Conclusions: Fusion rates varied greatly depending on the method used. Regardless of the measurement method, pseudarthrosis did not correlate with worse clinical outcome. The assessment of cervical alignment is highly depended of the method used. Cervical alignment using the Katsuura and Cobb methods correlated with clinical outcome and last follow up. Subsidence rates varied strongly depending on the measurement method used. Clinical outcome correlated with subsidence when the mochida assessment method is used.

Disclosures: Author 1: none; Author 2: none; Author 3: grants/research support: Relievant, Medtronic, Ulrich, Icotec, consultant: Relievant; Medtronic, Nexstim, Icotec, royalties: Spineart.

\section{QF75 \\ ARE THE RADIOGRAPHIC COMPONENTS OF THE AMES- ISSG CLASSIFICATION SPECIFIC TO CERVICAL DEFORMITY?}

Ziad Bakouny, Nour Khalil, Michel Salameh, Naji Bou Zeid, Renaud Lafage, Ismat Ghanem, Khalil Kharrat, Virginie Lafage, Gaby Kreichati, Ayman Assi

${ }^{1}$ Laboratory of Biomechanics and Medical Imaging, Faculty of Medicine, University of Saint-Joseph, Beirut, Lebanon; ${ }^{2}$ Spine division, Hospital for Special Surgery, New York, USA

Introduction: Adult spinal deformity (ASD) diagnosis and treatment have benefited from the classification devised by Schwab [1]. A similar classification has recently been proposed for cervical deformities and has been shown to be repeatable [2]. This classification includes three sagittal radiographic modifiers (radiographic chin brow vertical angle CBVA, T1slope-C2C7 T1S-CL, C2C7 SVA) stratified into 3 grades each, from grade 0 (no deformity) to 2 (maximal deformity). Despite the fact that normal cervical alignment is known to be heterogeneous [3], no study has investigated the distribution of the Ames-ISSG cervical classification modifiers in asymptomatic adult subjects. Our hypothesis was that grades 1 and 2 radiographic modifiers described by the Ames-ISSG cervical classification are found in asymptomatic adult subjects without alteration of quality of life (QoL).

Purpose: To determine whether grades 1 and 2 radiographic modifiers described by the Ames-ISSG cervical classification are found in asymptomatic adult subjects of varying ages.

Methods: 141 asymptomatic subjects [age $29.4 \pm 11$ years (18-59), 71F] with documented SF-36 QoL and full body biplanar X-rays performed in free standing position, were included. Head and cervical parameters were measured on lateral radiographs: CBVA, T1S-CL and $\mathrm{C} 2 \mathrm{C} 7 \mathrm{SVA}$. Subjects were classified according to the radiographic modifiers of the Ames-ISSG classification [2] and according to their age: $18-30$ years, $30-45$ years and $45-60$ years. The distribution of Ames-ISSG sagittal modifiers by age group was investigated and compared across age groups using Fischer's exact test (with calculation of Cramer'sV). Kruskal-Wallis or MannWhitney tests were used to compare SF-36 QoL components between the levels of each radiographic modifier.

Results: For the C2C7 SVA modifier, 99\% were classified as grade 0 and $1 \%$ as grade 1 . For the T1S-CL modifier, $11 \%$ were classified as grade $0,19 \%$ as grade 1 and $70 \%$ as grade 2 . For the CBVA modifier, $54 \%$ were classified as grade $0,43 \%$ as grade 1 and $3 \%$ as grade 2. In total, $96 \%$ of subjects had at least one modifier at grade 1 or 2 . There 
were significant relationships (Fig. 1) between age groups and grades of the T1S-CL $(p<0.001 ; \quad V=0.32)$ and CBVA modifiers $(\mathrm{p}=0.04 ; \mathrm{V}=0.22)$. All SF-36 components were similar between levels of both T1S-CL and CBVA modifiers ( $p>0.05$ ).

Conclusion: This is the first study to show that radiographic modifiers of the Ames-ISSG classification are very frequently encountered among asymptomatic adult subjects. Subjects with these radiographic anomalies did not have any alteration in QoL. While the T1S-CL modifier was more frequently encountered in younger subjects, the CBVA modifier was more prevalent in older subjects. This study suggests that radiographic modifiers of the Ames-ISSG classification are not specific to subjects with cervical deformities and can occur without alteration in QoL.

1) Schwab, Spine, 2012.

2) Ames, JNS spine, 2015.

3) Scheer, JNS spine, 2013

Disclosures: Author 1: none; Author 2: none; Author 3: none; Author 4: none; Author 5: none; Author 6: none; Author 7: none; Author 8: grants/research support: SRS, Stryker, DePuy Spine, K2M, NuVasive, consultant: NuVasive, stock/shareholder: Namaris INC, other financial report: DePuy Spine, NuVasive, K2M, MSD (speaking/teaching arrangements); Author 9: none; Author 10: none.

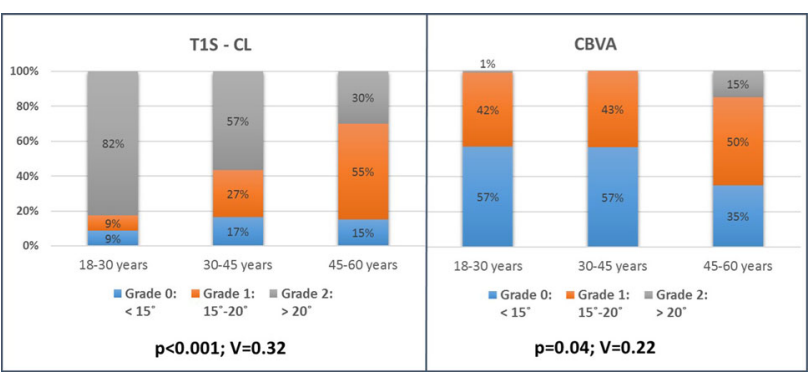

\section{QF76}

HOW CAN CERVICAL SAGITTAL ALIGNMENT PARAMETERS BE MEASURED ON X-RAY FILMS WHEN THE SHOULDER HIDES THE LOWER CERVICAL COLUMN?

Hitoshi Yamahata, Masanao Mori, Tadaaki Niiro, Jun Sugata, Kazunori Arita

Department of Neurosurgery, Kagoshima University, Kagoshima, Japan

Background: Measurement of cervical sagittal alignment is essential for a better understating of the patient condition. The T1 slope (T1S), the angle between the horizontal plane and the T1 superior endplate, has been reported to reflect cervical alignment and the global sagittal balance. However, when the cervicothoracic column is masked by the shoulder, measurements are difficult. We examined the angles between a horizontal line and the upper and lower endplate of $\mathrm{C} 7$ and C6 and assessed whether they were more useful than the T1S.

Methods: We included 189 patients ranging in age from 20 to 93 years who were seen at our institution. The T1S of all patients was measured on X-ray radiographs and compared with data obtained from adjacent levels. The angles between a horizontal line and the lower and upper endplate of C7 (C7LS and C7US) and C6 (C6LS and C6US) were measured to compare the T1S.

Results: The superior endplate of T1 was identified in 64 of 189 patients (34\%). The average T1S, C7LS, C7US, C6LS, and C6US was $24.2^{\circ} \pm 8.1^{\circ}, \quad 22.5^{\circ} \pm 9.1^{\circ}, \quad 21.2^{\circ} \pm 8.5^{\circ}, \quad 18.1^{\circ} \pm 8.9^{\circ}, \quad$ and $18.6^{\circ} \pm 8.5^{\circ}$, respectively. Except for C7LS $(\mathrm{p}=0.26)$, the difference of the mean between the T1S and the other parameters was statistically significant (unpaired t test, $\mathrm{p}<0.05$ ). There was a strong correlation between T1S and C7LS $(r=0.93)$, C7US $(r=0.91)$, C6LS $(r=0.87)$, and C6US $(r=0.81)$.

Conclusions: To measure the T1S on unclear X-rays, the C7 lower endplate, the closest endplate to $\mathrm{T} 1$, can be substituted for the $\mathrm{T} 1$ upper endplate. As the position of the endplate becomes higher, the correlation between the T1S and our other four parameters decreased for each Pearson correlation coefficient. This observation helps to predict cervical sagittal alignment by using the adjacent endplates in patients with unclear lower vertebral bodies.

Disclosures: Author 1: none; Author 2: none;Author 3: none; Author 4: none; Author 5: none.

QF77

SURGICAL CORRECTION FOR FIXED CERVICAL KYPHOTIC DEFORMITY. INDICATIONS, TECHNIQUES AND COMPLICATIONS IN A CONSECUTIVE SERIES OF 10 PATIENTS

John Michael Duff, Lukas Bobinski, Juan Barges-Coll

University Hospital of Lausanne, Lausanne, Switzerland

Introduction: Cervical kyphotic fixed deformity (CKFD) with myelopathy remains a significant challenge. Indications for surgery and surgical techniques are highly debatable and controversial. How CKFD fits into to the overall alignment of the spine remains to be fully elucidated.

We report the surgical results of symptomatic fixed cervical subaxial kyphotic deformity.

Materiels and methods: A retrospective chart review of ten patients who presented with symptomatic cervical compressive myelopathy, and a non-mobile cervical kyphosis was carried out. All patients were seen in clinical and radiological follow up. Follow up ranges from 6 to 24 months (mean 14.7). Patients were assessed with XR, and selectively with CT scan and MRI.

Results: Underlying pathlogies were degenerative (6), trauma (1), iatrogenic (1), NF 1 (1), and Down syndrome (1). There were 6 males and 4 females. Age range 46-75 (mean 60.5). Nurick pre op myelopathic scores 1-4 (mean 2.6). Preoperative C2-C7 Cobb angle ranged $1^{\circ}-33^{\circ}$ (mean $15.2^{\circ}$ ) of kyphosis.

All patients were operated using a back/front/back technique with neurophysiological intraoperative monitoring. First step is image-guided placement of posterior pedicle screws, followed by posterior release multilevel, bilateral Ponte-like cervical osteotomies, with no rod placement. Then, through an anterior approach, ventral release transuncal osteotomies and corpectomies are performed at multiple levels, with expandible cage placement to restore anterior column height. The posterior incision is reopened, lordotic rods are placed under compression to restore lordosis. Autologous bone graft isplaced posteriorly.

Early intraop and postoperative complications occurred in five patients (50\%), which included postoperative C6 weakness in 1 patient which required reoperation and screw replacement. A second patient had transient worsening of myelopathy which returned to baseline over several weeks. The remaining three patients had pneumonia, transient respiratory insufficience, and a superficial wound infection requiring revision.

Follow up Nurick scores improved in all but one patient who remained unchanged (mean 1.4). Bone fusion was documented in seven patients on CT scan. Three patients have a 6 month follow-up, 
so final fusion is not yet confirmed. There were no instrumentation failures at last follow-up. Follow-up kyphosis ranges from -1 to -35 (mean -8.9 ). Mean correction pre vs post op ranged from $5^{\circ}$ to $40^{\circ}$ (mean $23.9^{\circ}$ ).

Conclusion: Surgical correction of CKFD remains challenging. The high intraoperative/postoperative complication rate mirrors series published in the literature. However, surgical decompression and correction is probably the best current therapeutic option in patients with compressive cervical myelopathy in the context of a fixed kyphotic deformity, despite the complexity of the surgery and the high complication rate.

Disclosures: Author 1: stock/shareholder: KB Medical; Author 2: none; Author 3: consultant: J\&J.

\section{QF78 \\ OPTIMAL TIMING FOR TRAUMATIC CERVICAL SPINAL CORD INJURY WITH SURGICAL DECOMPRESSION: 10 YEARS CASES REVIEWED}

Dingjun Hao, Biao Wang, Hua Guo, Ziqi Zhu

Honghui Hospital Xi' an Jiaotong University College of Medicine, Xi' an, China

Purpose: Although there have been a lot of studies aimed at determining the effects and safety of early and late surgical decompression for traumatic cervical spinal cord injury, controversies still exist regarding the optimal timing of surgery for this serious trauma. This study was conducted to evaluate the effectiveness and safety of early and late surgical decompression for traumatic cervical spinal cord injury.

Methods: A retrospective cohort study was performed on consecutive patients who underwent surgical decompression for traumatic lower cervical spinal cord injury from January 2005 to January 2015. These patients were analysed according to the timing of surgical decompression. The early group comprised patients who underwent decompression within the first $72 \mathrm{~h}$ after being injured, whilst the late group comprised patients who underwent surgery after the first $72 \mathrm{~h}$. For analysis of neurologic improvement, patients who had completed a follow-up of at least 2 years were assessed. Surgical outcomes were analyzed based on intensive care unit and hospital stay, mortality rate, and complications.

Results: A total of 895 patients were identified, 738 cases $(82.5 \%)$ were data integrity and obtained follow-up, including 528 men and 210 women with an average age of 43.6 years, with 267 in the early group and 471 in the late group. Patients in both groups had made a significant neurologic improvement in the final follow-up, but no statistically significant difference was noted between two groups. No significant differences were identified between groups with regards complications (pneumonia, pulmonary embolism, wound infection, sepsis and urinary tract infection). Patients in the early group with a significantly shorter hospital stay of $14.9 \pm 3.2$ days compared to the late group of $20.6 \pm 4.2$ days $(\mathrm{p}<0.05)$. However, in terms of intensive care unit length of stay and ventilator days, no statistically significant difference in parameters was observed between groups.

Conclusion: As neurological recovery results in this study were similar between early and late decompression groups, early surgical intervention may not be helpful for improving neurologic recovery in traumatic cervical spinal cord injury. The manner and severity of spinal cord injury may have a significant effect on postoperative neurological recovery.

Keywords: Cervical spine, Spinal cord injury, Surgical treatment, Timing
Disclosures: Author 1: none; Author 2: none; Author 3: none; Author 4: none.

\section{QF79 \\ DOES CT ANGIOGRAPHY CHANGE THE MANAGEMENT OF PATIENTS WITH CERVICAL FRACTURES?}

Conor Dunn, Kimona Issa, Jeffrey Moore, Nancy Moontasri, Michael Faloon, Kumar Sinha, Ki Soo Hwang, Arash Emami

School of Health and Medical Sciences, Seton Hall University, South Orange, USA

Background: Specific cervical fractures in the trauma patient or neurologic deficits found on physical exam have been shown to be associated with vertebral artery injuries (VAIs). The role of computed tomography angiography (CTA) in these patients has been described by the prevalence of VAIs but not by the change in clinical management of these patients. In the interest of reducing the risk inferred upon the patient from CTA, cost savings and lifting time constraints for patients who are indicated for operative procedures, it is imperative to examine the role of CTA in the change of clinical management of the trauma patient with a cervical fracture. Here we determine if cervical fracture morphology can predict clinical management changes secondary to a CTA finding.

Study design: Clinical case series.

Methods: A retrospective review analyzed all patients with cervical spine fractures diagnosed on CT of the neck who underwent CTA between 2013 and 2016. CTA reports along with operative notes and all medical records of these patients were thoroughly reviewed. Those with positive findings of traumatic nature on CTA report who underwent change in clinical management were described and compared to those with negative findings. Patients who did not survive their hospital course were excluded from the study.

Results: Between 2013 and 2016111 patients underwent a CTA following $\mathrm{CT}$ of the head and neck demonstrating a cervical fracture. Of those 111, $21(18.9 \%)$ reports included positive findings of traumatic nature. Three $(2.7 \%)$ of those patients underwent surgical intervention secondary to these findings to address the VAIs. One patient had a comminuted fracture of $\mathrm{C} 2$, was found to have a dissection of the vertebral artery and underwent surgical intervention with stent placement. One patient had left C6 through T1 Facet and Transverse Process fractures, was found to have left vertebral artery laceration and underwent selective catheterization of the origin of the left vertebral artery. The final patient had fractures of the left C6-T1 Transverse Processes and left arm weakness on exam, was found to have a left vertebral artery laceration and underwent an open exploration and catheterization of the left vertebral artery. No patients had their medical management changed by positive finding on CTA.

Conclusions: Despite its common use in the work-up of the trauma patient with a cervical fracture, CTA rarely plays a role in changing the management of these patients. We found that only $3(2.7 \%)$ of the CTAs performed on patients with a cervical fracture changed clinical management. All 3 patients met previous literature recommendations to obtain CTA by either fracture pattern of neurologic deficit. These findings further suggest that, in the setting of the trauma patient with a cervical fracture, it is only necessary to obtain a CTA in those patients who have a neurologic deficit or fracture pattern previously described as high risk for VAIs.

Disclosures: Author 2: none; Author 3: none; Author 5: grants/research support: K2M, consultant: K2M, DePuys-Synthes; Author 6: none; Author 7: none; Author 8: grants/research support: Nuvasive, Depuy. 


\section{QF80 \\ REVISION SURGERY FOR CERVICAL CHORDOMAS IN PEDIATRIC PATIENTS}

Dezsoe Jeszenszky, Markus Loibl, Peter Obid, Christoph Heyde, Daniel Haschtmann, Frank Kleinstueck, Tamas F Fekete

Schulthess Clinic, Spine Center, Zürich, Switzerland

Introduction: Cervical chordomas are extremely rare primary tumours in paediatric patients. Few studies have reported on their treatment and outcome.

This retrospective case series reports on survival rate and complications of surgical treatment with piecemeal removal of chordoma remnants with/without tumour recurrence in the cervical spine of paediatric patients.

Materials and methods: The medical records, and pre- and postoperative radiological data of six paediatric patients with a cervical spine chordoma remnant or remnant-recurrence, treated surgically in our department between 2002 and 2016, were included in the study. All patients had undergone at least one previous surgery elsewhere, including intralesional or partial tumour-resection.

Results: The average time between the previous surgery and admission to our hospital was 6 months (range 2-10 months). The average age at revision was 9.6 years (range 5-16 years). Two chordomas were located at the craniocervical junction and four in the subaxial spine. All patients received adjuvant radiotherapy: 5 patients received proton beam radiation therapy (mean dose 72.8 CGE) and one patient, combined photon and local. The follow-up was on average 81.5 months (range 18-126 months). Analysis of postoperative MRIs and histopathological findings showed $\mathrm{R} 0$ resection in all cases. The following complications occurred: three patients had transient paresis postoperatively; one had a dehiscence of the pharyngeal wall, 8 years after surgery; one had an intraoperative CSF leak and due to repeated local recurrence of the tumour died 2 years after the first surgical revision. All other patients were free of tumour recurrence at the latest follow up (average 93.4 months (range 18-126 months)).

Discussion: Removal of a cervical spine chordoma is considered to be challenging, due to the complexity of the anatomy. Revision cases typically have a worse prognosis compared with those for whom it is the primary surgical treatment. Despite this, we showed that with a meticulous surgical technique and adjuvant radiation-therapy, a long disease-free survival can be achieved in children.

Conclusion: With thorough preoperative planning, appropriate surgical techniques, and adjuvant radiation therapy, tumour removal with a long disease-free survival can be achieved in most of these patients. Disclosures: Author 1: consultant: DePuy Synthes Spine, royalties: DePuy Synthes Spine; Author 2: none; Author 3: none; Author 4: royalties: Medacta International; Author 5: none; Author 6: grants/ research support: Depuy-Synthes; Author 7: none. 